\title{
On Ambrosetti-Malchiodi-Ni conjecture on two-dimensional smooth bounded domains
}

\author{
Suting Wei \\ School of Mathematics and Statistics, Central China Normal University, \\ Wuhan 430079, P. R. China. Email: stwei@mails.ccnu.edu.cn \\ Bin Xu \\ School of Mathematics and Statistics, Jiangsu Normal University, \\ Xuzhou, Jiangsu, 221116, P. R. China. Email: dream-010@163.com \\ Jun Yang * \\ School of Mathematics and Statistics \& Hubei Key Laboratory of Mathematical \\ Sciences, Central China Normal University, \\ Wuhan 430079, P. R. China. Email: jyang@mail.ccnu.edu.cn
}

\begin{abstract}
We consider the problem

$$
\epsilon^{2} \Delta u-V(y) u+u^{p}=0, \quad u>0 \quad \text { in } \quad \Omega, \quad \frac{\partial u}{\partial \nu}=0 \quad \text { on } \quad \partial \Omega,
$$

where $\Omega$ is a bounded domain in $\mathbb{R}^{2}$ with smooth boundary, the exponent $p>1, \epsilon>0$ is a small parameter, $V$ is a uniformly positive, smooth potential on $\bar{\Omega}$, and $\nu$ denotes the outward normal of $\partial \Omega$. Let $\Gamma$ be a curve intersecting orthogonally with $\partial \Omega$ at exactly two points and dividing $\Omega$ into two parts. Moreover, $\Gamma$ satisfies stationary and non-degeneracy conditions with respect to the functional $\int_{\Gamma} V^{\sigma}$, where $\sigma=\frac{p+1}{p-1}-\frac{1}{2}$. We prove the existence of a solution $u_{\epsilon}$ concentrating along the whole of $\Gamma$, exponentially small in $\epsilon$ at any positive distance from it, provided that $\epsilon$ is small and away from certain critical numbers. In particular, this establishes the validity of the two dimensional case of a conjecture by A. Ambrosetti, A. Malchiodi and W.-M. Ni(p.327, [4]).
\end{abstract}

Keywords: Ambrosetti-Malchiodi-Ni conjecture, concentration sets, Fermi coordinates

MSC 2010: 35J25, 35J61

\section{Introduction}

We consider the following problem for the existence of concentration phenomena

$$
\epsilon^{2} \Delta u-V(y) u+u^{p}=0, \quad u>0 \quad \text { in } \quad \Omega, \quad \frac{\partial u}{\partial \nu}=0 \quad \text { on } \quad \partial \Omega,
$$

\footnotetext{
*Corresponding author: Jun Yang, jyang@mail.ccnu.edu.cn
} 
where $\Omega$ is a bounded domain in $\mathbb{R}^{\mathbf{d}}$ with smooth boundary, $\epsilon>0$ is a small parameter, $\nu$ denotes the outward normal of $\partial \Omega$ and the exponent $p>1$.

\subsection{Case 1: $V \equiv 1$}

If $V \equiv 1$, problem (1.1) takes the form

$$
\epsilon^{2} \Delta u-u+u^{p}=0, \quad u>0 \quad \text { in } \quad \Omega, \quad \frac{\partial u}{\partial \nu}=0 \quad \text { on } \quad \partial \Omega,
$$

which is known as the stationary equation of Keller-Segel system in chemotaxis [42]. It can also be viewed as a limiting stationary equation of Gierer-Meinhardt system in biological pattern formation [30]. Problem (1.2) has been studied extensively in recent years. See [51] for backgrounds and references.

In the pioneering papers [42], [52]-[53], under the condition that $p$ is subcritical, i.e.,

$$
1<p<\frac{\mathbf{d}+2}{\mathbf{d}-2} \quad \text { when } \quad \mathbf{d} \geq 3 \quad \text { and } \quad 1<p<+\infty \quad \text { when } \quad \mathbf{d}=2,
$$

Lin, Ni and Takagi established, for $\epsilon$ sufficiently small, the existence of a least-energy solution $U_{\epsilon}$ of (1.2) with only one local maximum point $P_{\epsilon} \in \partial \Omega$. Moreover,

$$
H\left(P_{\epsilon}\right) \rightarrow \max _{P \in \partial \Omega} H(P) \quad \text { as } \quad \epsilon \rightarrow 0
$$

where $H(\cdot)$ is the mean curvature of $\partial \Omega$. Such a solution is called a boundary spike-layer.

Since then, many papers investigated further the solutions of (1.2) concentrating at one or multiple points of $\bar{\Omega}$. These solutions are called spike-layers. A general principle is that the location of interior spikes is determined by the distance function from the boundary. We refer the reader to the articles [11], [15], [22], [31]-[32], [58], and the references therein. On the other hand, boundary spikes are related to the mean curvature of $\partial \Omega$. This aspect was discussed in the papers [12], [14], [21], [34], [41], [57], [59], and the references therein. The coexistence of interior and boundary spikes was due to Gui and Wei [33]. A good review of the subject up to 2004 can be found in [51].

The question of constructing higher-dimensional concentration sets has been investigated only in recent years. It has been conjectured in [50] (see also [51]) by W.-M. Ni that: for any $1 \leq \mathbf{k} \leq \mathbf{d}-1$, problem (1.2) has a solution $U_{\epsilon}$ which concentrates on a $\mathbf{k}$-dimensional subset of $\bar{\Omega}$.

We mention some results for the existence of higher dimensional boundary concentration phenomena. In [48] and [49], Malchiodi and Montenegro considered (1.2) and made an initial and successful attempt to construct a solution $U_{\epsilon}$ with concentration at the boundary $\partial \Omega$ (or any component of $\partial \Omega$ ). In [46] and [47], Malchiodi showed the concentration phenomena for (1.2) along a closed non-degenerate geodesic of $\partial \Omega$ in the three dimensional smooth bounded domain $\Omega$. Mahmoudi and Malchiodi [43] proved a full general concentration of solutions along $\mathbf{k}$-dimensional $(1 \leq \mathbf{k} \leq \mathbf{d}-1)$ non-degenerate minimal submanifolds of the boundary for $\mathbf{d} \geq 3$ and $1<p<(\mathbf{d}-\mathbf{k}+2) /(\mathbf{d}-\mathbf{k}-2)$.

On the other hand, in order to verify the two dimensional case of the conjecture, Wei and Yang $[61,62]$ considered problem (1.2) with solutions concentrating on curves near a non-degenerate 
line $\Gamma^{\prime}$ connecting the boundary of $\Omega$ at right angle. There are also some other results $[6,7,24$, $25,26]$ to exhibit concentration phenomena on interior line segments connecting the boundary of $\Omega$. For higher dimensional extension, the reader can refer to $[8,16,35,40]$. In [16], Dancer and Yan constructed solutions of (1.2) concentrating on higher dimensional subsets inside the domain and on the boundary of the domain separately. In [40] Li, Peng and Yan showed the existence of solutions concentrating simultaneously on several higher dimensional interior and boundary manifolds. The reader can also refer to the survey paper by Wei [60].

\subsection{Case 2: $V \not \equiv$ constant}

In this case, we first concern the problem on the whole space, i.e.,

$$
\epsilon^{2} \Delta u-V(y) u+u^{p}=0, \quad u>0 \quad \text { in } \mathbb{R}^{\mathbf{d}},
$$

where $\epsilon>0$ is a small parameter, the exponent $p>1$, and $V$ is a smooth function with

$$
\inf _{y \in \mathbb{R}^{\mathbf{d}}} V(y)>0 .
$$

In the pioneering work [29], Floer and Weinstein constructed positive solutions to this problem when $p=3$ and $\mathbf{d}=1$ with concentration taking place near a given point $y_{0}$ with $V^{\prime}\left(y_{0}\right)=$ $0, V^{\prime \prime}\left(y_{0}\right) \neq 0$, being exponentially small in $\epsilon$ outside any neighborhood of $y_{0}$. This result has been subsequently extended to higher dimensions to the construction of solutions exhibiting concentration around one or more points of space under various assumptions on the potential and the nonlinearity by many authors. We refer the reader for instance to $[2,5,13,17,18,19,20,28]$ and the references therein.

An important question is whether solutions exhibiting concentration on higher dimensional set exists. In [3], Ambrosetti, Malchiodi and Ni considered the case of $V=V(|y|)$ and constructed radial solutions $u_{\epsilon}(|y|)$ exhibiting concentration on a sphere $|y|=r_{0}$ in the form

$$
u_{\epsilon}(r) \sim V^{\frac{1}{p-1}}\left(r_{0}\right) w\left(V^{\frac{1}{2}}\left(r_{0}\right) \epsilon^{-1}\left(r-r_{0}\right)\right), \quad r=|y|
$$

under the assumption that $r_{0}>0$ is a non-degenerate critical point of

$$
M(r)=r^{n-1} V^{\sigma}(r),
$$

where $w$ is the unique (even) solution of

$$
w^{\prime \prime}-w+w^{p}=0, \quad w>0, \quad w^{\prime}(0)=0, \quad w( \pm \infty)=0,
$$

and

$$
\sigma=\frac{p+1}{p-1}-\frac{1}{2} .
$$

Based on heuristic arguments, in 2003 A. Ambrosetti, A. Malchiodi and W.-M. Ni raised the following conjecture (p.465, [3]): Let $\mathcal{K}$ be a non-degenerate $\mathbf{k}$-dimensional stationary manifold of the following functional

$$
\int_{\mathcal{K}} V^{\frac{p+1}{p-1}-\frac{1}{2}(\mathbf{d}-\mathbf{k})}
$$

Then there exists a solution to (1.3) concentrating near $\mathcal{K}$, at least along a subsequence $\epsilon_{j} \rightarrow 0$. 
For $\mathbf{d}=2, \mathbf{k}=1$, del Pino, Kowalczyk and Wei [23] proved the validity of this conjecture under some gap condition. Recently, Mahmoudi, Malchiodi and Montenegro [44] established the validity of the above conjecture in the case of $\mathbf{d}=3, \mathbf{k}=1$. They also considered the complex solutions of (1.3) carrying momentums. For the concentration phenomena directed along general manifolds of codimension one, see [56]. For a more general problem with suitable hypotheses for the potentials, Bartsch and Peng constructed spike layered solution which concentrates on certain $\mathbf{d}-1$ dimensional spheres in $[9,10]$.

Let us go back to the problem on smooth bounded domain with homogeneous Neumann boundary condition, i.e., problem (1.1) with the assumption that $V$ is a smooth function and

$$
\inf _{y \in \bar{\Omega}} V(y)>0 .
$$

Ambrosetti, Malchioci and Ni [4] imposed the following hypotheses on the function $V$ :

(V1). $V \in C^{1}\left(\mathbb{R}^{+}, \mathbb{R}\right)$

(V2). $V$ is bounded and satisfies (1.8),

and introduced the auxiliary potential

$$
M(r)=r^{\mathbf{d}-1} V^{\sigma}(r), \quad \text { where } \sigma=\frac{p+1}{p-1}-\frac{1}{2} .
$$

By taking advantage of the boundary effect, they showed the existence of interior concentration for radial symmetric solutions to (1.1). One of their results is as follows: Let $\Omega \subseteq \mathbb{R}^{\mathbf{d}}$ be the ball $B_{1}$ and $p>1$. Suppose that the function $M$ satisfies the condition

$$
M^{\prime}(1)>0
$$

Then there exists a family of radial solutions $u_{\epsilon}$ of (1.1) concentrating on $|y|=r_{\epsilon}$, where $r_{\epsilon}$ is a local maximum for $u_{\epsilon}$ such that $1-r_{\epsilon} \sim \epsilon|\log \epsilon|$. To the best knowledge of the authors, for (1.1), there are no results on the interior non-radial and symmetric concentration phenomena(without intersection between $\partial \Omega$ and the concentration set).

For problem (1.1), another natural case is the existence of interior concentration phenomena intersecting the boundary of $\Omega$. This is the conjecture also by A. Ambrosetti, A. Malchiodi and W.-M. Ni in 2004 (p.327, [4]), which can be stated as: Let $\mathcal{K}$ be a k-dimensional manifold intersecting $\partial \Omega$ perpendicularly, which is also stationary and non-degenerate with respect to the following functional

$$
\int_{\mathcal{K}} V^{\frac{p+1}{p-1}-\frac{1}{2}(\mathbf{d}-\mathbf{k})}
$$

Then there exists a solution to (1.1) concentrating near $\mathcal{K}$, at least along a subsequence $\epsilon_{j} \rightarrow 0$.

\subsection{Main results}

In the present paper, to avoid complicated computation, we will consider (1.1) and provide an affirmative answer to the last-mentioned conjecture(called Ambrosetti-Malchiodi-Ni conjecture) only in the case: $\mathbf{d}=2$ and $\mathbf{k}=1$. It should be mentioned that the potential term $V$ and the boundary of $\Omega$ will play an important role in the sense that they basically determine the location of the concentration set and also bring much more difficulties in our procedure of the constructing solutions due to the intersection between $\partial \Omega$ and the concentration set. The new 
ingredient is to set up a new local coordinate system such that we can decompose the interaction among the interior concentration layers, the boundary of $\Omega$ and the potential term $V$.

More precisely, in the present paper, for the existence of a solution $u_{\epsilon}$ concentrating along a curve, say $\Gamma$, we make the following assumptions on $\Gamma$ :

(A1). $\Gamma$ is a curve intersecting $\partial \Omega$ at exactly two points, saying $P_{1}, P_{2}$, and, at these points $\Gamma \perp \partial \Omega$. In the small neighborhoods of $P_{1}, P_{2}$, the boundary $\partial \Omega$ are two curves, say $\mathcal{C}_{1}$ and $\mathcal{C}_{2}$, which can be represented by the graphs of two functions respectively

$$
\begin{aligned}
& y_{2}=\varphi_{1}\left(y_{1}\right) \quad \text { with }\left(0, \varphi_{1}(0)\right)=P_{1}, \\
& y_{2}=\varphi_{2}\left(y_{1}\right) \text { with }\left(0, \varphi_{2}(0)\right)=P_{2} .
\end{aligned}
$$

Moreover, after rescaling, we can always assume that $|\Gamma|=1$ and then denote $k$ the curvature of $\Gamma$, also $k_{1}, k_{2}$ the signed curvatures of $\mathcal{C}_{1}$ at $P_{1}$ and $\mathcal{C}_{2}$ at $P_{2}$.

(A2). The curve $\Gamma$ is a non-degenerate geodesic relative to the weighted arc length $\int_{\Gamma} V^{\sigma}$, where $\sigma$ is defined in (1.9). This will be clarified in the next section, see (2.9) and (2.11).

By recalling $w$ given in (1.6), we consider the associated linearized eigenvalue problem,

$$
h^{\prime \prime}-h+p w^{p-1} h=\lambda h \quad \text { in } \mathbb{R}, \quad h( \pm \infty)=0 .
$$

It is well known that this equation possesses a unique positive eigenvalue $\lambda_{0}$ in $H^{1}(\mathbb{R})$, with associated eigenfunction $Z$ (even and positive) which can be normalized so that $\int_{\mathbb{R}} Z^{2}=1$. In fact, a simple computation shows that

$$
\lambda_{0}=\frac{1}{4}(p-1)(p+3), \quad Z=\frac{1}{\sqrt{\int_{\mathbb{R}} w^{p+1}}} w^{\frac{p+1}{2}} .
$$

By setting

$$
\lambda_{*}=\frac{\lambda_{0} \ell^{2}}{\pi^{2}} \quad \text { with } \quad \ell=\int_{\Gamma} V^{\frac{1}{2}}
$$

we can formulate our main result.

Theorem 1.1. Let $\mathbf{d}=2$ and $\Gamma$ be a non-degenerate, stationary curve for the weighted length functional $\int_{\Gamma} V^{\sigma}$, as described above. For given $c>0$ there exists $\epsilon_{0}>0$ such that for all $\epsilon<\epsilon_{0}$ satisfying the gap condition

$$
\left|\epsilon^{2} j^{2}-\lambda_{*}\right| \geq c \epsilon, \quad \forall j \in \mathbb{N},
$$

problem (1.1) has a positive solution $u_{\epsilon}$, which, for $y$ given by (2.4) in the neighborhood of $\Gamma$, takes the form

$$
u_{\epsilon}(y)=V(0, \theta)^{\frac{1}{p-1}} w\left(V(0, \theta)^{\frac{1}{2}} \frac{t}{\epsilon}\right)(1+o(1)) .
$$

For some number $c_{0}>0, u_{\epsilon}$ satisfies globally

$$
u_{\epsilon}(y) \leq \exp \left(-c_{0} \epsilon^{-1} \operatorname{dist}(y, \Gamma)\right)
$$


Here are some words for further discussion. We do not prove the result for all small values $\epsilon>$ 0 but only for those which lie away from certain critical numbers due to the resonance phenomena. In fact, the gap condition (1.14) will be useful in dealing with the resonance phenomenon, which was described in Section 1 of [23].

For the convenience of expression in the procedure to show the validity of Theorem 1.1, by the rescaling

$$
y=\epsilon \tilde{y}
$$

in $\mathbb{R}^{2},(1.1)$ will be rewritten as

$$
\Delta u_{\tilde{y}}-V(\epsilon \tilde{y}) u+u^{p}=0 \quad \text { in } \Omega_{\epsilon}, \quad \frac{\partial u}{\partial \nu_{\epsilon}}=0 \quad \text { on } \partial \Omega_{\epsilon},
$$

where $\Omega_{\epsilon}=\Omega / \epsilon$ and then also $\Gamma_{\epsilon}=\Gamma / \epsilon$. In order to decompose the interaction among concentration layers, the potential $V$ and the boundary $\partial \Omega_{\epsilon}$, and then construct good approximations to real solutions, we will first set up a local coordinate system to present $y$ and then $\tilde{y}$ by the relation (1.16), called modified Fermi coordinates, in Section 2. This local coordinate system helps us set up the stationary and non-degeneracy conditions for $\Gamma$, see (2.9) and (2.11).

An outline of proof for Theorem 1.1 will be given in Section 3. Gluing procedure from [23] will be applied to transform (1.17) into a projected form of a local problem, see (3.56)-(3.59) in Section 3, which will be solved in Sections 5.1 and 5.2. Note that the resolution theory of (3.56)-(3.59) relies heavily on the properties of the local approximate solution (cf. (4.70)), which will be constructed in Section 4 step by step in such a way that it solves the nonlinear problem locally up to order $O\left(\epsilon^{2}\right)$.

To get a real solution, the well-known infinite dimensional reduction method [23] will be needed in Sections 6-7. In fact, the reduced problem inherits the resonance phenomena and will be handled by complicated Fourier analysis with the help of the gap condition in (1.14). Some tedious computation will be given in Appendices A and B.

\section{Geometric preliminaries}

In this section, inspired by [55] we will set up a local coordinate system in the neighborhood of $\Gamma$, called modified Fermi coordinates, and then derive its asymptotic expansion. The notion of stationary and non-degenerate curve $\Gamma$ will be also derived in the last part of this section.

\subsection{Modified Fermi Coordinates}

Recall the assumptions (A1) and (A2) in Section 1 and the notation therein. For basic notions of curves, such as the signed curvature, the reader can refer to the book by do Carmo [27]. The local coordinate system can be given as the following:

Step 1. Let the natural parameterization of the curve $\Gamma$ be as follows:

$$
\gamma_{0}:[0,1] \rightarrow \Gamma \subset \bar{\Omega} \subset \mathbb{R}^{2} .
$$

We can extend $\gamma_{0}$ slightly in a smooth way, i.e., for some small positive numbers $\sigma_{0}$, and define the mapping

$$
\gamma:\left(-\sigma_{0}, 1+\sigma_{0}\right) \rightarrow \mathbb{R}^{2},
$$


such that

$$
\gamma(\tilde{\theta})=\gamma_{0}(\tilde{\theta}), \quad \forall \tilde{\theta} \in[0,1]
$$

There holds the Frenet formula

$$
\gamma^{\prime \prime}=k n, \quad n^{\prime}=-k \gamma^{\prime},
$$

where $k, n$ are the curvature and the normal of $\gamma$. Choosing $\delta_{0}>0$ very small, and setting

$$
\mathfrak{S}_{1} \equiv\left(-\delta_{0}, \delta_{0}\right) \times\left(-\sigma_{0}, 1+\sigma_{0}\right),
$$

we construct the following mapping

$$
\mathbb{H}: \mathfrak{S}_{1} \rightarrow \mathbb{H}\left(\mathfrak{S}_{1}\right) \equiv \Omega_{\delta_{0}, \sigma_{0}} \quad \text { with } \quad \mathbb{H}(\tilde{t}, \tilde{\theta})=\gamma(\tilde{\theta})+\tilde{t} n(\tilde{\theta}) .
$$

Note that $\mathbb{H}$ is a diffeomorphism (locally) and $\mathbb{H}(0, \tilde{\theta})=\gamma(\tilde{\theta})$.

Step 2. Denote the preimage

$$
\tilde{\mathcal{C}}_{1} \equiv \mathbb{H}^{-1}\left(\mathcal{C}_{1}\right), \quad \tilde{\mathcal{C}}_{2} \equiv \mathbb{H}^{-1}\left(\mathcal{C}_{2}\right),
$$

which can be parameterized respectively by $\left(\tilde{t}, \tilde{\varphi}_{1}(\tilde{t})\right)$ and $\left(\tilde{t}, \tilde{\varphi}_{2}(\tilde{t})\right)$ for some smooth functions $\tilde{\varphi}_{1}(\tilde{t}), \tilde{\varphi}_{2}(\tilde{t})$, and then define a mapping

$$
\tilde{\mathbb{H}}: \mathfrak{S}_{1} \rightarrow \mathfrak{S}_{2} \equiv \tilde{\mathbb{H}}\left(\mathfrak{S}_{1}\right) \subset \mathbb{R}^{2},
$$

in the form

$$
t=\tilde{t}, \quad \theta=\frac{\tilde{\theta}-\tilde{\varphi}_{1}(\tilde{t})}{\tilde{\varphi}_{2}(\tilde{t})-\tilde{\varphi}_{1}(\tilde{t})} .
$$

This transformation will straighten up the curves $\tilde{\mathcal{C}}_{1}$ and $\tilde{\mathcal{C}}_{2}$.

It is obvious that

$$
\tilde{\mathbb{H}}^{-1}(0, \theta)=(0, \theta), \theta \in[0,1] .
$$

Moreover, we have

Lemma 2.1. There hold

$$
\tilde{\varphi}_{1}^{\prime}(0)=0, \quad \tilde{\varphi}_{2}^{\prime}(0)=0 .
$$

Then, there exist the relations

$$
k_{1}=\tilde{\varphi}_{1}^{\prime \prime}(0), \quad k_{2}=\tilde{\varphi}_{2}^{\prime \prime}(0) .
$$

The proof of this lemma will be given in Appendix A.

Step 3. We define the modified Fermi coordinates

$$
y=F(t, \theta)=\mathbb{H} \circ \tilde{\mathbb{H}}^{-1}(t, \theta):\left(-\delta_{0}, \delta_{0}\right) \times\left(-\sigma_{0}, 1+\sigma_{0}\right) \rightarrow \mathbb{R}^{2}
$$

for the given small positive constants $\sigma_{0}$ and $\delta_{0}$. More precisely,

$$
\begin{aligned}
F(t, \theta) & =\mathbb{H}\left(t, \quad\left(\tilde{\varphi}_{2}(t)-\tilde{\varphi}_{1}(t)\right) \theta+\tilde{\varphi}_{1}(t)\right) \\
& =\gamma\left(\left(\tilde{\varphi}_{2}(t)-\tilde{\varphi}_{1}(t)\right) \theta+\tilde{\varphi}_{1}(t)\right)+\operatorname{tn}\left(\left(\tilde{\varphi}_{2}(t)-\tilde{\varphi}_{1}(t)\right) \theta+\tilde{\varphi}_{1}(t)\right) .
\end{aligned}
$$


For convenience's sake, in the following, we also denote

$$
\Theta(t, \theta) \equiv\left(\tilde{\varphi}_{2}(t)-\tilde{\varphi}_{1}(t)\right) \theta+\tilde{\varphi}_{1}(t)
$$

and so that

$$
F(t, \theta)=\gamma(\Theta(t, \theta))+\operatorname{tn}(\Theta(t, \theta))
$$

Note also that

$$
\begin{aligned}
\Theta(0, \theta) & =\theta, \quad \Theta_{t}(0, \theta)=0, \quad \Theta_{\theta}(0, \theta)=1, \\
\Theta_{t t}(0, \theta) & =\left(k_{2}-k_{1}\right) \theta+k_{1}, \quad \Theta_{t t \theta}(0, \theta)=k_{2}-k_{1},
\end{aligned}
$$

due to (2.2) and (2.3). These quantities will play an important role in the further settings.

The asymptotic expressions of this coordinate system will be given by the following basic facts.

Lemma 2.2. The mapping $F$ in (2.5) has the following properties:

(1). $F(0, \theta)=\gamma(\theta)$,

(2). $\frac{\partial F}{\partial t}(0, \theta)=n(\theta)$,

(3). $q_{1}(\theta) \equiv \frac{\partial^{2} F}{\partial t^{2}}(0, \theta)=\gamma^{\prime}(\theta) \cdot \Theta_{t t}(0, \theta) \quad \perp n(\theta)$,

(4). $q_{2}(\theta) \equiv \frac{\partial^{3} F}{\partial t^{3}}(0, \theta)=\gamma^{\prime}(\theta) \cdot \Theta_{t t t}(0, \theta)+3 n^{\prime}(\theta) \cdot \Theta_{t t}(0, \theta) \quad \perp n(\theta)$.

The proof of this lemma will be given in Appendix A.

\subsection{Stationary and non-degenerate curves}

Finally, for a curve $\Gamma$ connecting the boundary $\partial \Omega$, we will make precise the concept of being non-degenerate geodesic for the metric $\mathrm{d} s^{2}=V^{\sigma}\left(\mathrm{d} y_{1}^{2}+\mathrm{d} y_{2}^{2}\right)$ where $\sigma$ is given in (1.9). Consider the deformation of $\Gamma$ along the normal with end points staying on $\partial \Omega$, i.e.,

$$
\Gamma_{\mathfrak{g}}: \gamma(\Theta(\mathfrak{g}(\theta), \theta))+\mathfrak{g}(\theta) n(\Theta(\mathfrak{g}(\theta), \theta)) .
$$

Taking the functional defined on $\mathfrak{g}$ as

$$
\begin{aligned}
\mathcal{J}(\mathfrak{g}) & =\int_{0}^{1} V^{\sigma}\left(\Gamma_{\mathfrak{g}}(\theta)\right)\left|\Gamma_{\mathfrak{g}}^{\prime}(\theta)\right| \mathrm{d} \theta \\
& =\int_{0}^{1} V^{\sigma}\left[(1-k \mathfrak{g})^{2}\left(\Theta_{t} \mathfrak{g}^{\prime}+\Theta_{\theta}\right)^{2}+\left(\mathfrak{g}^{\prime}\right)^{2}\right]^{\frac{1}{2}} \mathrm{~d} \theta,
\end{aligned}
$$

where $\Theta=\Theta(\mathfrak{g}(\theta), \theta)$.

We write $V(y)=V(t, \theta)$ in the modified Fermi coordinates by setting

$$
t=\mathfrak{g}(\theta),\left.\quad V(y)\right|_{\Gamma_{\mathfrak{g}}}=V(\mathfrak{g}(\theta), \theta) .
$$


The curve $\Gamma$ is said to be stationary for the weighted length $\int_{\Gamma_{\mathfrak{g}}} V^{\sigma}$ if the first variation of $\mathcal{J}$ at $\mathfrak{g}=0$ is equal to zero. That is, for any smooth function $h(\theta)$ defined at $[0,1]$

$$
\begin{aligned}
0=\mathcal{J}^{\prime}(0)[h]= & \int_{0}^{1} V^{\sigma}(0, \theta) \Theta_{\theta}^{-1}\left[(-k h) \Theta_{\theta}^{2}+\Theta_{\theta}\left(\Theta_{t} h^{\prime}+\Theta_{\theta t} h\right)\right] \mathrm{d} \theta \\
& +\int_{0}^{1}\left(V^{\sigma}\right)_{t} \cdot h \cdot \Theta_{\theta} \mathrm{d} \theta
\end{aligned}
$$

which is equivalent to the relation

$$
\sigma V_{t}(0, \theta)=k(\theta) V(0, \theta)
$$

We assume the validity of this relation at $\Gamma$ and then consider the second variation of $\mathcal{J}$

$$
\begin{aligned}
\mathcal{J}^{\prime \prime}(0)[h, f]= & \left.\frac{\mathrm{d}}{\mathrm{d} s}\right|_{s=0} \mathcal{J}^{\prime}(0+s f)[h] \\
= & \int_{0}^{1} V^{\sigma}\left(\Theta_{t t} f+f^{\prime}\right) h^{\prime} \mathrm{d} \theta+\int_{0}^{1} V^{\sigma} \Theta_{t t} \cdot f^{\prime} h \mathrm{~d} \theta \\
& +\int_{0}^{1}\left[\left(V^{\sigma}\right)_{t t}-2 k\left(V^{\sigma}\right)_{t}+\Theta_{t t \theta} V^{\sigma}\right] f h \mathrm{~d} \theta \\
= & \left.h \cdot V^{\sigma}\left(\Theta_{t t} f+f^{\prime}\right)\right|_{0} ^{1}+\int_{0}^{1}\left(-V^{\sigma} f^{\prime \prime}-\sigma V^{\sigma-1} V_{\theta} f^{\prime}\right) h \mathrm{~d} \theta \\
& +\int_{0}^{1}\left[-\sigma V^{\sigma-1} V_{\theta} \Theta_{t t}+\left(V^{\sigma}\right)_{t t}-2 k\left(V^{\sigma}\right)_{t}\right] f h \mathrm{~d} \theta .
\end{aligned}
$$

The curve $\Gamma$ is said to be nondegenerate if

$$
\mathcal{J}^{\prime \prime}(0)[h, f]=0, \quad \forall h \in H^{1}(0,1),
$$

then $f \equiv 0$. Using (2.9), it is easy to check that the relation (2.10) is equivalent to that the boundary problem

$$
\begin{gathered}
f^{\prime \prime}+\sigma V^{-1} V_{\theta} f^{\prime}+\left[\sigma V^{-1} V_{\theta} \Theta_{t t}-\sigma V^{-1} V_{t t}+\left(1+\frac{1}{\sigma}\right) k^{2}\right] f=0 \quad \text { in }(0,1), \\
f^{\prime}(1)+k_{2} f(1)=0, \quad f^{\prime}(0)+k_{1} f(0)=0
\end{gathered}
$$

has only the trivial solution.

\section{Outline of the proof}

In this section, the strategy to prove Theorem 1.1 will be provided step by step.

\subsection{The gluing procedure}

For any given approximate solution $\mathbf{W}$ (to be chosen later, cf. (4.71)) and a perturbation term $\Phi$ defined on $\Omega_{\epsilon}$, the function $u(\tilde{y})=\mathbf{W}(\tilde{y})+\Phi(\tilde{y})$ satisfies (1.17) if and only if

$$
\mathbf{L}(\Phi)=-\mathbf{E}-\mathbf{N}(\Phi) \text { in } \Omega_{\epsilon},
$$


with boundary condition

$$
\frac{\partial \Phi}{\partial \nu_{\epsilon}}+\frac{\partial \mathbf{W}}{\partial \nu_{\epsilon}}=0 \quad \text { on } \partial \Omega_{\epsilon}
$$

where

$$
\begin{gathered}
\mathbf{L}(\Phi)=\Delta_{\tilde{y}} \Phi-V(\epsilon \tilde{y}) \Phi+p \mathbf{W}^{p-1} \Phi, \quad \mathbf{E}=\Delta_{\tilde{y}} \mathbf{W}-V(\epsilon \tilde{y}) \mathbf{W}+\mathbf{W}^{p} \\
\mathbf{N}(\Phi)=(\mathbf{W}+\Phi)^{p}-\mathbf{W}^{p}-p \mathbf{W}^{p-1} \Phi .
\end{gathered}
$$

We separate $\Phi$ in the following form

$$
\Phi(\tilde{y})=\eta_{3 \delta}^{\epsilon}(s) \check{\phi}(\tilde{y})+\check{\psi}(\tilde{y}),
$$

where $s$ is the normal coordinate to $\Gamma_{\epsilon}$. In the above formula, the cut-off function is defined as

$$
\eta_{3 \delta}^{\epsilon}(s)=\eta_{3 \delta}(\epsilon|s|),
$$

where $\eta_{\delta}(t)$ is also a smooth cut-off function defined as

$$
\eta_{\delta}(t)=1, \quad \forall 0 \leq t \leq \delta \quad \text { and } \quad \eta(t)=0, \quad \forall t>2 \delta,
$$

for any fixed number $\delta<\delta_{0} / 100$, where $\delta_{0}>0$ is a small constant in (2.4). With this definition, $\Phi$ is a solution of (3.1)-(3.2) if the pair $(\check{\phi}, \check{\psi})$ satisfies the following coupled system:

$$
\begin{aligned}
\eta_{3 \delta}^{\epsilon}\left(\Delta_{\tilde{y}} \check{\phi}-V \check{\phi}+p \mathbf{W}^{p-1} \check{\phi}\right)= & \eta_{\delta}^{\epsilon}\left[-\mathbf{N}\left(\eta_{3 \delta}^{\epsilon} \check{\phi}+\check{\psi}\right)-\mathbf{E}-p \mathbf{W}^{p-1} \check{\psi}\right] \quad \text { in } \Omega_{\epsilon}, \\
\Delta_{\tilde{y}} \check{\psi}-V \check{\psi}+\left(1-\eta_{\delta}^{\epsilon}\right) p \mathbf{W}^{p-1} \check{\psi}= & -\epsilon^{2}\left(\Delta_{\tilde{y}} \eta_{3 \delta}^{\epsilon}\right) \check{\phi}-2 \epsilon\left(\nabla_{\tilde{y}} \eta_{3 \delta}^{\epsilon}\right)\left(\nabla_{\tilde{y}} \check{\phi}\right) \\
& -\left(1-\eta_{\delta}^{\epsilon}\right) \mathbf{N}\left(\eta_{3 \delta}^{\epsilon} \check{\phi}+\check{\psi}\right)-\left(1-\eta_{\delta}^{\epsilon}\right) \mathbf{E} \quad \text { in } \Omega_{\epsilon},
\end{aligned}
$$

with the boundary conditions

$$
\begin{gathered}
\eta_{3 \delta}^{\epsilon} \frac{\partial \check{\phi}}{\partial \nu_{\epsilon}}+\eta_{\delta}^{\epsilon} \frac{\partial \mathbf{W}}{\partial \nu_{\epsilon}}=0 \quad \text { on } \partial \Omega_{\epsilon}, \\
\frac{\partial \check{\psi}}{\partial \nu_{\epsilon}}+\left(1-\eta_{\delta}^{\epsilon}\right) \frac{\partial \mathbf{W}}{\partial \nu_{\epsilon}}+\epsilon \frac{\partial \eta_{3 \delta}^{\epsilon}}{\partial \nu_{\epsilon}} \check{\phi}=0 \quad \text { on } \partial \Omega_{\epsilon} .
\end{gathered}
$$

Given a small $\check{\phi}$, we first solve problem (3.6) and (3.8) for $\check{\psi}$. Assume now that $\check{\phi}$ satisfies the following decay property

$$
|\nabla \check{\phi}(\tilde{y})|+|\check{\phi}(\tilde{y})| \leq C e^{-\gamma / \epsilon} \quad \text { if } \operatorname{dist}\left(\tilde{y}, \Gamma_{\epsilon}\right)>\delta / \epsilon,
$$

for a certain constant $\gamma>0$, also that

$$
\mathbf{W}(\tilde{y}) \text { is exponentially small if } \operatorname{dist}\left(\tilde{y}, \Gamma_{\epsilon}\right)>\delta / \epsilon \text {. }
$$

These two assumptions will be fulfilled by the choosing of $\phi$ in Proposition 5.2 due to the relation (3.25), and also $\mathbf{W}$ in (4.71). The solvability can be done in the following way: let us observe that $s$ is the normal coordinate to $\Gamma_{\epsilon}$. Then the problem

$$
\begin{array}{lc}
\Delta \check{\psi}-\left[V-\left(1-\eta_{\delta}^{\epsilon}\right) p \mathbf{W}^{p-1}\right] \check{\psi}=h & \text { in } \Omega_{\epsilon}, \\
\frac{\partial \check{\psi}}{\partial \nu_{\epsilon}}=-\left(1-\eta_{\delta}^{\epsilon}\right) \frac{\partial \mathbf{W}}{\partial \nu_{\epsilon}}-\epsilon \frac{\partial \eta_{3 \delta}^{\epsilon}}{\partial \nu_{\epsilon}} \check{\phi} & \text { on } \partial \Omega_{\epsilon},
\end{array}
$$


has a unique bounded solution $\check{\psi}$ whenever $\|h\|_{\infty}<+\infty$. Moreover,

$$
\|\check{\psi}\|_{\infty} \leq C\|h\|_{\infty} .
$$

Since $\mathbf{N}$ is power-like with power greater than one, a direct application of contraction mapping principle yields that (3.6) and (3.8) has a unique (small) solution $\check{\psi}=\check{\psi}(\check{\phi})$ with

$$
\|\check{\psi}(\check{\phi})\|_{L^{\infty}} \leq C \epsilon\left[\|\check{\phi}\|_{L^{\infty}(|s|>\delta / \epsilon)}+\|\nabla \check{\phi}\|_{L^{\infty}(|s|>\delta / \epsilon)}+e^{-\delta / \epsilon}\right],
$$

where $|s|>\delta / \epsilon$ denotes the complement in $\Omega_{\epsilon}$ of $\delta / \epsilon$-neighborhood of $\Gamma_{\epsilon}$. Moreover, the nonlinear operator $\check{\psi}$ satisfies a Lipschitz condition of the form

$$
\left\|\check{\psi}\left(\check{\phi}_{1}\right)-\check{\psi}\left(\check{\phi}_{2}\right)\right\|_{L^{\infty}} \leq C \epsilon\left[\left\|\check{\phi}_{1}-\check{\phi}_{2}\right\|_{L^{\infty}(|s|>\delta / \epsilon)}+\left\|\nabla \check{\phi}_{1}-\nabla \check{\phi}_{2}\right\|_{L^{\infty}(|s|>\delta / \epsilon)}\right] .
$$

The key observation is that, after solving (3.6) and (3.8), we can concern (3.5) and (3.7) as a local nonlinear problem involving $\check{\psi}=\check{\psi}(\check{\phi})$, which can be solved in local coordinates in the sense that we can decompose the interaction among the boundary, the concentration set and the potential $V$, and then construct a good approximate solution and also derive the resolution theory of the nonlinear problem by delicate analysis. This procedure is called a gluing technique in $[23]$.

\subsection{Local formulation of the problem}

As described in the above, the next step is to consider (3.5)-(3.7) in the neighbourhood of $\Gamma_{\epsilon}$ so that by the relation $\tilde{y}=y / \epsilon$ in (1.16) close to $\Gamma_{\epsilon}$, the variables $y$ can be represented by the modified Fermi coordinates $(t, \theta)$ in $(2.4)$, which have been set up in Section 2 .

\subsubsection{Local form of the problem}

By using the local coordinates $(t, \theta)$ in $(2.4)$, the local forms of the differential operators $\triangle_{y}$ and $\partial / \partial \nu$ in (1.1) are given in (B.4), (B.7) and (B.10). By recalling the rescaling $y=\epsilon \tilde{y}$ in (1.16), it is useful to locally introduce change of variables

$$
u(y)=\hat{u}(\tilde{y}) \quad \text { and } \quad \epsilon s=t, \epsilon z=\theta .
$$

Then (B.4) will give

$$
\epsilon^{2} \Delta_{y}=\epsilon^{2} \partial_{t t}^{2}+\epsilon^{2} \partial_{\theta \theta}^{2}+\epsilon^{2} \bar{B}_{1}(\cdot)+\epsilon^{2} \bar{B}_{0}(\cdot),
$$

where the form of $\bar{B}_{1}$ in (B.5) will provide

$$
\begin{aligned}
\epsilon^{2} \bar{B}_{1}(\cdot) & =-\epsilon^{2}\left(k+k^{2} t\right) \partial_{t}-2 \epsilon^{2} t \varpi \partial_{t \theta}^{2}-\epsilon^{2} \varpi \partial_{\theta} \\
& =-\left(\epsilon k+\epsilon^{2} k^{2} s\right) \partial_{s}-2 \epsilon s \varpi \partial_{s z}^{2}-\epsilon \varpi \partial_{z} \\
& \equiv \hat{B}_{1}(\cdot),
\end{aligned}
$$

and the expression of $\bar{B}_{0}$ in (B.6) indicates that

$$
\begin{aligned}
\epsilon^{2} \bar{B}_{0}(\cdot) & =2 \epsilon^{2} k t \partial_{\theta \theta}^{2}+a_{1} \epsilon^{2} t^{2} \partial_{\theta \theta}^{2}+a_{2} \epsilon^{2} t^{3} \partial_{t t}^{2}+a_{3} \epsilon^{2} t^{2} \partial_{t \theta}^{2}+a_{4} \epsilon^{2} t^{2} \partial_{t}+a_{5} \epsilon^{2} t \partial_{\theta} \\
& =2 k \epsilon s \partial_{z z}^{2}+a_{1} \epsilon^{2} s^{2} \partial_{z z}^{2}+a_{2} \epsilon^{3} s^{3} \partial_{s s}^{2}+a_{3} \epsilon^{2} s^{2} \partial_{s z}^{2}+a_{4} \epsilon^{3} s^{2} \partial_{s}+a_{5} \epsilon^{2} s \partial_{z} \\
& \equiv \hat{B}_{0}(\cdot) .
\end{aligned}
$$


In the above formulas, the smooth functions $\varpi, a_{1}, \cdots, a_{5}$ are given in (B.2) and (B.3). This implies that

$$
\Delta_{\tilde{y}}=\epsilon^{2} \Delta_{y}=\partial_{s s}^{2}+\partial_{z z}^{2}+\hat{B}_{1}(\cdot)+\hat{B}_{0}(\cdot) .
$$

Similarly, the normal derivative $\partial / \partial \nu_{\epsilon}$ can be derived from (B.7) and (B.10). Indeed, if $z=0$, it becomes the following boundary operator

$$
\mathbb{D}_{0} \equiv k_{1} \epsilon s \partial_{s}+b_{1} \epsilon^{2} s^{2} \partial_{s}-\partial_{z}-k(0) \epsilon s \partial_{z}+b_{2} \epsilon^{2} s^{2} \partial_{z}+\hat{D}_{0}^{0}(\cdot),
$$

where

$$
\hat{D}_{0}^{0}=\epsilon \bar{D}_{0}^{0}
$$

And, at $z=1 / \epsilon$, it has the form

$$
\mathbb{D}_{1} \equiv k_{2} \epsilon s \partial_{s}+b_{3} \epsilon^{2} s^{2} \partial_{s}-\partial_{z}-k(1) \epsilon s \partial_{z}+b_{4} \epsilon^{2} s^{2} \partial_{z}+\hat{D}_{0}^{1}(\cdot),
$$

where

$$
\hat{D}_{0}^{1}=\epsilon \bar{D}_{0}^{1} .
$$

In the above equations, the constants $b_{1}, b_{2}, b_{3}, b_{4}$ are given in (B.9) and (B.12).

Hence, the equation in (3.5) can be locally recast in $(s, z)$ coordinate system as follows

$$
\eta_{3 \delta}^{\epsilon} \check{L}(\check{\phi})=\eta_{\delta}^{\epsilon}\left[-\mathbf{N}\left(\eta_{3 \delta}^{\epsilon} \check{\phi}+\check{\psi}\right)-\mathbf{E}-p \mathbf{W}^{p-1} \check{\psi}\right],
$$

where the linear operator is

$$
\check{L}(\check{\phi})=\check{\phi}_{s s}+\check{\phi}_{z z}+\hat{B}_{1}(\check{\phi})+\hat{B}_{0}(\check{\phi})-V(\epsilon s, \epsilon z) \check{\phi}+p \mathbf{W}^{p-1} \check{\phi}
$$

and the error is now locally expressed in the form

$$
\mathbf{E}=\mathbf{W}_{s s}+\mathbf{W}_{z z}+\hat{B}_{1}(\mathbf{W})+\hat{B}_{0}(\mathbf{W})-V(\epsilon s, \epsilon z) \mathbf{W}+\mathbf{W}^{p} .
$$

The boundary condition in (3.7) can also be expressed precisely in the local coordinates. If $z=0$,

$$
\eta_{3 \delta}^{\epsilon} \mathbb{D}_{0}(\check{\phi})=-\eta_{\delta}^{\epsilon} \mathbf{G}_{0} \quad \text { with } \quad \mathbf{G}_{0}=\mathbb{D}_{0}(\mathbf{W}),
$$

and also, at $z=1 / \epsilon$ there holds

$$
\eta_{3 \delta}^{\epsilon} \mathbb{D}_{1}(\check{\phi})=-\eta_{\delta}^{\epsilon} \mathbf{G}_{1} \quad \text { with } \quad \mathbf{G}_{1}=\mathbb{D}_{1}(\mathbf{W}) .
$$

\subsubsection{Further changing of variables}

In this section we consider a further change of variables in equation (3.17) in such a way that it replaces at main order the potential $V$ by 1 .

Let

$$
\alpha(\theta)=V(0, \theta)^{\frac{1}{p-1}}, \quad \beta(\theta)=V(0, \theta)^{\frac{1}{2}},
$$

and fix two twice differentiable functions $f$ and $h$, which satisfy the following constraints

$$
\|f\|_{*} \equiv\|f\|_{L^{\infty}(0,1)}+\left\|f^{\prime}\right\|_{L^{\infty}(0,1)}+\left\|f^{\prime \prime}\right\|_{L^{2}(0,1)} \leq \epsilon^{1 / 2},
$$


and

$$
h^{\prime}(1)+k_{2} h(1)=0, \quad h^{\prime}(0)+k_{1} h(0)=0 .
$$

In fact, $f$ will be found by the reduction method described in Sections 6-7, while $h$ is a solution to problem (4.69). By defining the relation

$$
\check{\phi}(s, z)=\alpha(\epsilon z) \phi(x, z) \quad \text { with } \quad x=\beta(\epsilon z)(s-f(\epsilon z)-h(\epsilon z)),
$$

we can express equation (3.17) in terms of these new coordinates due to the following trivial computation

$$
\begin{aligned}
\check{\phi}_{s}= & \alpha \beta \phi_{x}, \quad \check{\phi}_{s s}=\alpha \beta^{2} \phi_{x x}, \\
\check{\phi}_{z}= & \epsilon \alpha^{\prime} \phi+\alpha \phi_{x}(\beta(s-f-h))_{z}+\alpha \phi_{z}, \\
\check{\phi}_{z z}= & \epsilon^{2} \alpha^{\prime \prime} \phi+2 \epsilon \alpha^{\prime}\left[\phi_{x}(\beta(s-f-h))_{z}+\phi_{z}\right] \\
& +\alpha\left[\phi_{x x}\left|(\beta(s-f-h))_{z}\right|^{2}+2 \phi_{x z}(\beta(s-f-h))_{z}+\phi_{x}(\beta(s-f-h))_{z z}+\phi_{z z}\right], \\
\check{\phi}_{s z}= & \epsilon \alpha^{\prime} \beta \phi_{x}+\epsilon \alpha \beta^{\prime} \phi_{x}+\alpha \beta \phi_{x x}(\beta(s-f-h))_{z}+\alpha \beta \phi_{x z},
\end{aligned}
$$

where

$$
\begin{aligned}
(\beta(s-f-h))_{z} & =\epsilon\left[\beta^{\prime}(s-f-h)-\beta f^{\prime}-\beta h^{\prime}\right]=\epsilon \beta^{-1} \beta^{\prime} x-\epsilon \beta f^{\prime}-\epsilon \beta h^{\prime}, \\
(\beta(s-f-h))_{z z} & =\epsilon^{2}\left[\beta^{\prime \prime}(s-f-h)-2 \beta^{\prime} f^{\prime}-2 \beta^{\prime} h^{\prime}-\beta f^{\prime \prime}-\beta h^{\prime \prime}\right] \\
& =\epsilon^{2} \beta^{-1} \beta^{\prime \prime} x-2 \epsilon^{2} \beta^{\prime} f^{\prime}-2 \epsilon^{2} \beta^{\prime} h^{\prime}-\epsilon^{2} \beta f^{\prime \prime}-\epsilon^{2} \beta h^{\prime \prime} .
\end{aligned}
$$

In order to write down the equation, it is also convenient to expand

$$
V(\epsilon s, \epsilon z)=V(0, \epsilon z)+V_{t}(0, \epsilon z) \cdot \epsilon s+\frac{1}{2} V_{t t}(0, \epsilon z) \cdot \epsilon^{2} s^{2}+a_{6}(\epsilon s, \epsilon z) \epsilon^{3} s^{3}
$$

for a smooth function $a_{6}(t, \theta)$.

By the above change of coordinates, the linear operator $\check{L}$ has the following form

$$
\begin{aligned}
\check{L}(\check{\phi})= & \alpha \beta^{2} \phi_{x x}+\epsilon^{2} \alpha^{\prime \prime} \phi+2 \epsilon \alpha^{\prime}\left[\epsilon\left(\frac{\beta^{\prime}}{\beta} x-\beta f^{\prime}-\beta h^{\prime}\right) \phi_{x}+\phi_{z}\right] \\
& +\alpha\left[\epsilon^{2}\left|\frac{\beta^{\prime}}{\beta} x-\beta f^{\prime}-\beta h^{\prime}\right|^{2} \phi_{x x}+2 \epsilon\left(\frac{\beta^{\prime}}{\beta} x-\beta f^{\prime}-\beta h^{\prime}\right) \phi_{x z}\right. \\
& \left.+\epsilon^{2}\left(\frac{\beta^{\prime \prime}}{\beta} x-2 \beta^{\prime} f^{\prime}-2 \beta^{\prime} h^{\prime}-\beta f^{\prime \prime}-\beta h^{\prime \prime}\right) \phi_{x}+\phi_{z z}\right]-\left[\epsilon k+\epsilon^{2} k^{2}\left(\frac{x}{\beta}+f+h\right)\right] \alpha \beta \phi_{x} \\
& -2 \epsilon \varpi\left(\frac{x}{\beta}+f+h\right)\left[\epsilon \alpha^{\prime} \beta \phi_{x}+\epsilon \alpha \beta^{\prime} \phi_{x}+\epsilon \alpha \beta^{\prime} x \phi_{x x}-\epsilon \alpha \beta^{2}\left(f^{\prime}+h^{\prime}\right) \phi_{x x}+\alpha \beta \phi_{x z}\right] \\
& -\epsilon \varpi\left[\epsilon \alpha^{\prime} \phi+\epsilon \alpha\left(\frac{\beta^{\prime}}{\beta} x-\beta f^{\prime}-\beta h^{\prime}\right) \phi_{x}+\alpha \phi_{z}\right]+\hat{B}_{0}(\check{\phi}) \\
& -\left[V(0, \epsilon z)+V_{t}(0, \epsilon z) \cdot \epsilon s+\frac{1}{2} V_{t t}(0, \epsilon z) \epsilon^{2} s^{2}+\epsilon^{3} a_{6}(\epsilon s, \epsilon z) s^{3}\right] \alpha \phi+p \mathbf{W}^{p-1} \alpha \phi .
\end{aligned}
$$


Dividing the above equation by $\alpha \beta^{2}$, we can induce that

$$
\bar{L}(\phi) \equiv\left(\alpha \beta^{2}\right)^{-1} \tilde{L}(\check{\phi})=\beta^{-2} \phi_{z z}+\phi_{x x}-\phi+\beta^{-2} p \mathbf{W}^{p-1} \phi+B_{3}(\phi),
$$

where $B_{3}(\phi)$ is a linear differential operator defined by

$$
\begin{aligned}
B_{3}(\phi)= & -\beta^{-1}\left[\epsilon k+\epsilon^{2} k^{2}\left(\frac{x}{\beta}+f+h\right)\right] \phi_{x} \\
+ & \beta^{-2}\left[\epsilon^{2}\left|\frac{\beta^{\prime}}{\beta} x-\beta f^{\prime}-\beta h^{\prime}\right|^{2} \phi_{x x}+2 \epsilon\left(\frac{\beta^{\prime}}{\beta} x-\beta f^{\prime}-\beta h^{\prime}\right) \phi_{x z}\right. \\
& \left.\quad+\epsilon^{2}\left(\frac{\beta^{\prime \prime}}{\beta} x-2 \beta^{\prime} f^{\prime}-2 \beta^{\prime} h^{\prime}-\beta f^{\prime \prime}-\beta h^{\prime \prime}\right) \phi_{x}\right] \\
& +\frac{\epsilon^{2}}{\alpha \beta^{2}} \alpha^{\prime \prime} \phi+\frac{2 \epsilon \alpha^{\prime}}{\alpha \beta^{2}}\left[\epsilon\left(\frac{\beta^{\prime}}{\beta} x-\beta f^{\prime}-\beta h^{\prime}\right) \phi_{x}+\phi_{z}\right] \\
- & \frac{2 \epsilon \varpi}{\alpha \beta^{2}}\left(\frac{x}{\beta}+f+h\right)\left[\epsilon \alpha^{\prime} \beta \phi_{x}+\epsilon \alpha \beta^{\prime} \phi_{x}+\epsilon \alpha \beta^{\prime} x \phi_{x x}-\epsilon \alpha \beta^{2} f^{\prime} \phi_{x x}\right. \\
& -\frac{\epsilon \varpi}{\alpha \beta^{2}}\left[\epsilon \alpha^{\prime} \phi+\epsilon \alpha\left(\frac{\beta^{\prime}}{\beta} x-\beta f^{\prime}-\beta h^{\prime}\right) \phi_{x}+\alpha \phi_{z}\right] \\
- & {\left[\epsilon \sigma^{-1} k\left(\frac{x}{\beta}+f+h\right)+\frac{\epsilon^{2}}{2} \beta^{-2} V_{t t}\left(\frac{x}{\beta}+f+h\right)^{2}\right] \phi+B_{2}(\phi), }
\end{aligned}
$$

where $\varpi$ is defined in (B.2). In the last line of (3.29), we have used the relation (2.9) given by the assumption that $\Gamma$ is stationary. Here

$$
B_{2}(\phi)=\left(\alpha \beta^{2}\right)^{-1} \hat{B}_{0}(\check{\phi})+\left(\alpha \beta^{2}\right)^{-1} a_{6}(\epsilon s, \epsilon z) \epsilon^{3} s^{3} \alpha \phi,
$$

and $\hat{B}_{0}(\check{\phi})$ is the operator in (3.14) where derivatives are expressed in terms of formulas through (3.26), $a_{6}$ is given by (3.27), and $s$ is replaced by $\beta^{-1} x+f+h$.

In the coordinates $(x, z)$, the boundary conditions in (3.20) and (3.21) can be recast as follows. For $z=0$,

$$
\eta_{3 \delta}^{\epsilon}\left(D_{3}^{0}(\phi)-\phi_{z}+D_{0}^{0}(\phi)\right)=-\alpha^{-1} \eta_{\delta}^{\epsilon} \mathbf{G}_{0}
$$

where

$$
\begin{aligned}
D_{3}^{0}(\phi)= & \epsilon\left[b_{5} x+\beta\left(k_{1} f+f^{\prime}\right)\right] \phi_{x}-\epsilon \alpha^{\prime} \alpha^{-1} \phi-\epsilon k\left(\frac{x}{\beta}+f+h\right) \phi_{z} \\
& +\epsilon^{2}\left[b_{1}\left(\frac{x}{\beta}+f+h\right)^{2} \beta-k\left(\frac{x}{\beta}+f+h\right)\left(\frac{\beta^{\prime}}{\beta} x-\beta f^{\prime}-\beta h^{\prime}\right)\right] \phi_{x} \\
& -\epsilon^{2} \frac{k \alpha^{\prime}}{\alpha}\left(\frac{x}{\beta}+f+h\right) \phi+\epsilon^{2} b_{2}\left(\frac{x}{\beta}+f+h\right)^{2} \phi_{z},
\end{aligned}
$$

the constant $b_{5}$ is

$$
b_{5}=k_{1}-\frac{\beta^{\prime}(0)}{\beta(0)}
$$


and

$$
\begin{aligned}
D_{0}^{0}(\phi)= & \alpha^{-1} \hat{D}_{0}^{0}(\check{\phi})+\epsilon^{3} b_{2}\left(\frac{x}{\beta}+f+h\right)^{2} \frac{\alpha^{\prime}}{\alpha} \phi \\
& +\epsilon^{3} b_{2} \phi_{x}\left(\frac{x}{\beta}+f+h\right)^{2}\left(\frac{\beta^{\prime}}{\beta} x-\beta f^{\prime}-\beta h^{\prime}\right) .
\end{aligned}
$$

Similarly, for $z=1 / \epsilon$, we have

$$
\eta_{3 \delta}^{\epsilon}\left(D_{3}^{1}(\phi)-\phi_{z}+D_{0}^{1}(\phi)\right)=-\alpha^{-1} \eta_{\delta}^{\epsilon} \mathbf{G}_{1},
$$

where

$$
\begin{aligned}
D_{3}^{1}(\phi)= & \epsilon\left[b_{6} x+\beta\left(k_{2} f+f^{\prime}\right)\right] \phi_{x}-\epsilon \alpha^{\prime} \alpha^{-1} \phi-\epsilon k\left(\frac{x}{\beta}+f+h\right) \phi_{z} \\
& +\epsilon^{2}\left[b_{3}\left(\frac{x}{\beta}+f+h\right)^{2} \beta-k\left(\frac{x}{\beta}+f+h\right)\left(\frac{\beta^{\prime}}{\beta} x-\beta f^{\prime}-\beta h^{\prime}\right)\right] \phi_{x} \\
& -\epsilon^{2} \frac{k \alpha^{\prime}}{\alpha}\left(\frac{x}{\beta}+f+h\right) \phi+\epsilon^{2} b_{4}\left(\frac{x}{\beta}+f+h\right)^{2} \phi_{z},
\end{aligned}
$$

the constant

$$
b_{6}=k_{2}-\frac{\beta^{\prime}(1)}{\beta(1)}
$$

and

$$
\begin{aligned}
D_{0}^{1}(\phi)= & \alpha^{-1} \hat{D}_{0}^{1}(\check{\phi})+\epsilon^{3} b_{4}\left(\frac{x}{\beta}+f+h\right)^{2} \frac{\alpha^{\prime}}{\alpha} \phi \\
& +\epsilon^{3} b_{4} \phi_{x}\left(\frac{x}{\beta}+f+h\right)^{2}\left(\frac{\beta^{\prime}}{\beta} x-\beta f^{\prime}-\beta h^{\prime}\right) .
\end{aligned}
$$

As a conclusion, it is derived that (3.5) and (3.7) becomes, in local coordinates $(x, z)$,

$$
\begin{gathered}
\eta_{3 \delta}^{\epsilon} \bar{L}(\phi)=\left(\alpha \beta^{2}\right)^{-1} \eta_{\delta}^{\epsilon}\left[-\mathbf{N}\left(\eta_{3 \delta}^{\epsilon} \check{\phi}+\check{\psi}\right)-\mathbf{E}-p \mathbf{W}^{p-1} \check{\psi}\right], \\
\eta_{3 \delta}^{\epsilon}\left(D_{3}^{0}(\phi)-\phi_{z}+D_{0}^{0}(\phi)\right)=-\alpha^{-1} \eta_{\delta}^{\epsilon} \mathbf{G}_{0}, \\
\eta_{3 \delta}^{\epsilon}\left(D_{3}^{1}(\phi)-\phi_{z}+D_{0}^{1}(\phi)\right)=-\alpha^{-1} \eta_{\delta}^{\epsilon} \mathbf{G}_{1} .
\end{gathered}
$$

\subsection{Derivation of the projected problem by extension method}

Before going further to the resolution theory of (3.39)-(3.41), we give some notation.

Notation: For simplicity, denote

$$
\mathcal{F}=\{(f, e) \mid \text { the functions } f \text { and e satisfy (3.23) and (3.50) respectively }\} .
$$

Observe that all functions involved are expressed in $(x, z)$-variables, and the natural domain for those variables can be extended to the infinite strip

$$
\begin{aligned}
\mathcal{S} & =\{(x, z):-\infty<x<\infty, \quad 0<z<1 / \epsilon\}, \\
\partial_{0} \mathcal{S} & =\{(x, z):-\infty<x<\infty, \quad z=0\}, \\
\partial_{1} \mathcal{S} & =\{(x, z):-\infty<x<\infty, \quad z=1 / \epsilon\} .
\end{aligned}
$$


Accordingly, we define

$$
\begin{aligned}
\hat{\mathcal{S}} & =\{(x, \tilde{z}):-\infty<x<\infty, \quad 0<\tilde{z}<\ell / \epsilon\}, \\
\partial_{0} \hat{\mathcal{S}} & =\{(x, \tilde{z}):-\infty<x<\infty, \quad \tilde{z}=0\}, \\
\partial_{1} \hat{\mathcal{S}} & =\{(x, \tilde{z}):-\infty<x<\infty, \quad \tilde{z}=\ell / \epsilon\},
\end{aligned}
$$

where $\ell$ is a constant defined in (1.13).

One of the left jobs is to find the local forms of the approximate solution $\mathbf{W}$ with the constraint (3.10) and also of the error E. We recall the transformation in (3.22) and (3.25), and then define for the local form of $\mathbf{W}$ by the relation

$$
\eta_{10 \delta}^{\epsilon}(s) \mathbf{W}=\eta_{3 \delta}^{\epsilon}(s) \alpha(\epsilon z) v(x, z) \quad \text { with } \quad x=\beta(\epsilon z)(s-f(\epsilon z)-h(\epsilon z)) .
$$

As we have done for the equation (3.17), $\mathbf{E}$ can be locally recast in $(x, z)$ coordinate system by the relation

$$
\left(\alpha \beta^{2}\right)^{-1} \eta_{\delta}^{\epsilon}(s) \mathbf{E}=\eta_{\delta}^{\epsilon}(s) \mathcal{E},
$$

where

$$
\mathcal{E}=S(v) \quad \text { with } \quad S(v) \equiv \beta^{-2} v_{z z}+v_{x x}-v+v^{p}+B_{3}(v),
$$

with the operator $B_{3}$ defined in (3.29). In the coordinates $(x, z)$, the boundary errors can be recast as follows. For $z=0$, there holds

$$
\alpha^{-1} \eta_{\delta}^{\epsilon}(s) \mathbf{G}_{0}=\eta_{\delta}^{\epsilon}(s) g_{0} \quad \text { with } \quad g_{0}=D_{3}^{0}(v)-v_{z}+D_{0}^{0}(v),
$$

and also for $z=1 / \epsilon$, we have

$$
\alpha^{-1} \eta_{\delta}^{\epsilon}(s) \mathbf{G}_{1}=\eta_{\delta}^{\epsilon}(s) g_{1} \quad \text { with } \quad g_{1}=D_{3}^{1}(v)-v_{z}+D_{0}^{1}(v) .
$$

It is of importance that (3.46), (3.48) and (3.49) hold only in a small neighbourhood of $\Gamma_{\epsilon}$. Hence we will consider $v, \mathcal{E}$ as functions of the variables $x$ and $z$ on $\mathcal{S}$, and also $g_{0}, g_{1}$ on $\partial_{0} \mathcal{S}$ and $\partial_{1} \mathcal{S}$ in the sequel. We will find $v=v_{5}$ in (4.70) step by step in Section 4 , so that $\mathbf{W}$ will be given in (4.71) with the property in (3.10). In fact, to deal with the resonance, in addition to the parameters $f$ and $h$, we shall add one more parameter, say $e$ (cf. (4.35)), in the approximate solution $v$. In all what follows, we will assume the validity of the following constraint on the parameter $e$ :

$$
\|e\|_{* *} \equiv\|e\|_{L^{\infty}(0,1)}+\epsilon\left\|e^{\prime}\right\|_{L^{2}(0,1)}+\epsilon^{2}\left\|e^{\prime \prime}\right\|_{L^{2}(0,1)} \leq \epsilon^{1 / 2} .
$$

The exact forms of the error terms $\mathcal{E}, g_{0}$ and $g_{1}$ will be given in (4.72) and (4.73).

Then define an operator on the whole strip $\mathcal{S}$ in the form

$$
\begin{aligned}
\mathcal{L}(\phi) \equiv & \beta^{-2} \phi_{z z}+\phi_{x x}-\phi+p w^{p-1} \phi+\chi(\epsilon|x|) B_{3}(\phi) \\
& +p\left(\beta^{-2} \chi(\epsilon|x|) \mathbf{W}^{p-1}-w^{p-1}\right) \phi \quad \text { in } \mathcal{S},
\end{aligned}
$$

and also the operators

$$
\mathcal{D}_{1}(\phi)=\chi(\epsilon|x|) D_{3}^{1}(\phi)-\phi_{z}+\chi(\epsilon|x|) D_{0}^{1}(\phi) \text { on } \partial_{1} \mathcal{S},
$$




$$
\mathcal{D}_{0}(\phi)=\chi(\epsilon|x|) D_{3}^{0}(\phi)-\phi_{z}+\chi(\epsilon|x|) D_{0}^{0}(\phi) \quad \text { on } \partial_{0} \mathcal{S},
$$

where $\chi(r)$ is a smooth cut-off function which equals 1 for $0 \leq r<10 \delta$ that vanishes identically for $r>20 \delta$. For the local form of the nonlinear part, we have

$$
\eta_{\delta}^{\epsilon}\left(\alpha \beta^{2}\right)^{-1}\left[\mathbf{N}\left(\eta_{3 \delta}^{\epsilon} \check{\phi}+\check{\psi}\right)+p \mathbf{W}^{p-1} \check{\psi}\right]=\eta_{\delta}^{\epsilon} \mathcal{N}(\phi),
$$

by the notation

$$
\mathcal{N}(\phi)=\left(v_{5}+\phi+\psi\right)^{p}-v_{5}^{p}-p v_{5}^{p-1} \phi,
$$

where in the right hand side the term $\psi$ is transformed from $\check{\psi}$ by the relation (3.25). Rather than solving problem (3.39)-(3.41) directly, we deal with the following projected problem: for each pair of parameters $f$ and $e$ in $\mathcal{F}$, finding functions $\phi \in H^{2}(\mathcal{S}), c, d \in L^{2}(0,1)$ and constants $l_{0}, l_{1}, m_{0}, m_{1}$ such that

$$
\begin{aligned}
\mathcal{L}(\phi)= & \eta_{\delta}^{\epsilon}(s)[-\mathcal{E}-\mathcal{N}(\phi)]+c(\epsilon z) \chi(\epsilon|x|) w_{x}+d(\epsilon z) \chi(\epsilon|x|) Z \quad \text { in } \mathcal{S}, \\
& \mathcal{D}_{1}(\phi)=-\eta_{\delta}^{\epsilon}(s) g_{1}+l_{1} \chi(\epsilon|x|) w_{x}+m_{1} \chi(\epsilon|x|) Z \quad \text { on } \partial_{1} \mathcal{S}, \\
& \mathcal{D}_{0}(\phi)=-\eta_{\delta}^{\epsilon}(s) g_{0}+l_{0} \chi(\epsilon|x|) w_{x}+m_{0} \chi(\epsilon|x|) Z \quad \text { on } \partial_{0} \mathcal{S}, \\
& \int_{\mathbb{R}} \phi(x, z) w_{x}(x) \mathrm{d} x=\int_{\mathbb{R}} \phi(x, z) Z(x) \mathrm{d} x=0, \quad 0<z<\frac{1}{\epsilon} .
\end{aligned}
$$

In Proposition 5.2, we will prove that this problem has a unique solution $\phi$ so that $\check{\phi}$ satisfies the constraint (3.9) due the relation (3.25).

After this has been done, our task is to adjust the parameters $f$ and $e$ by the reduction method such that the functions $c$ and $d$ are identically zero, and the constants $l_{1}, l_{0}, m_{1}$ and $m_{0}$ are zero too. By the estimates in Section 6 , it is equivalent to solving a nonlocal, nonlinear coupled second order system of differential equations for the pair $(f, e)$ with suitable boundary conditions. In section 7 , we will prove this system is solvable in $\mathcal{F}$.

\section{The local approximate solutions}

The main objective of this section is to construct the approximation $v$ and then evaluate its error terms $\mathcal{E}, g_{0}$ and $g_{1}$ in the coordinate system $(x, z)$.

\subsection{The first approximate solution}

Let $w(x)$ denote the unique positive solution of (1.6). Then, we take

$$
v_{1}(x, z)=w(x)
$$

as the first approximate solution. For further improvements, it is of importance to evaluate the errors by plugging the approximate solution to (3.47), (3.48) and (3.49).

We first consider $S\left(v_{1}\right)$ and identify the terms of order $\epsilon$ and those of order $\epsilon^{2}$ :

$$
S\left(v_{1}\right)=S(w)=B_{3}(w)
$$




$$
\begin{aligned}
= & -\beta^{-1}\left[\epsilon k+\epsilon^{2} k^{2}\left(\frac{x}{\beta}+f+h\right)\right] w_{x} \\
& +\beta^{-2}\left[\epsilon^{2}\left|\frac{\beta^{\prime}}{\beta} x-\beta f^{\prime}-\beta h^{\prime}\right|^{2} w_{x x}+\epsilon^{2}\left(\frac{\beta^{\prime \prime}}{\beta} x-2 \beta^{\prime} f^{\prime}-2 \beta^{\prime} h^{\prime}-\beta f^{\prime \prime}-\beta h^{\prime \prime}\right) w_{x}\right] \\
& +\frac{\epsilon^{2}}{\alpha \beta^{2}} \alpha^{\prime \prime} w+\frac{2 \epsilon \alpha^{\prime}}{\alpha \beta^{2}}\left[\epsilon\left(\frac{\beta^{\prime}}{\beta} x-\beta f^{\prime}-\beta h^{\prime}\right) w_{x}\right] \\
& -\frac{2 \epsilon \varpi}{\alpha \beta^{2}}\left(\frac{x}{\beta}+f+h\right)\left[\epsilon \alpha^{\prime} \beta w_{x}+\epsilon \alpha \beta^{\prime} w_{x}+\epsilon \alpha \beta^{\prime} x w_{x x}-\epsilon \alpha \beta^{2} f^{\prime} w_{x x}-\epsilon \alpha \beta^{2} h^{\prime} w_{x x}\right] \\
& -\frac{\epsilon \varpi}{\alpha \beta^{2}}\left[\epsilon \alpha^{\prime} w+\epsilon \alpha\left(\frac{\beta^{\prime}}{\beta} x-\beta f^{\prime}-\beta h^{\prime}\right) w_{x}\right] \\
& -\left[\epsilon \sigma^{-1} k\left(\frac{x}{\beta}+f+h\right)+\frac{\epsilon^{2}}{2 \beta^{2}} V_{t t}\left(\frac{x}{\beta}+f+h\right)^{2}\right] w+B_{2}(w) .
\end{aligned}
$$

Here $B_{2}(w)$ turned out to be of size $\epsilon^{3}$. Gathering terms of order $\epsilon$ and $\epsilon^{2}$, we get

$$
\begin{aligned}
S(w)= & -\epsilon \beta^{-1}\left[k w_{x}+\sigma^{-1} k x w\right]-\epsilon \sigma^{-1} k(f+h) w \\
& -\epsilon^{2}\left[\frac{k^{2}}{\beta} f w_{x}+\frac{f^{\prime \prime}}{\beta} w_{x}+\frac{2 \beta^{\prime}}{\beta^{2}} f^{\prime} w_{x}+\frac{2 \alpha^{\prime}}{\alpha \beta} f^{\prime} w_{x}+\frac{2 \beta^{\prime}}{\beta^{2}} f^{\prime} x w_{x x}+\frac{V_{t t}}{\beta^{3}} f x w\right] \\
& +\epsilon^{2}\left(f^{\prime 2} w_{x x}+2 f^{\prime} h^{\prime} w_{x x}-\frac{1}{2} \beta^{-2} V_{t t} f^{2} w+2 \varpi f f^{\prime} w_{x x}+2 \varpi f^{\prime} h w_{x x}\right) \\
& -\frac{2 \epsilon^{2} \varpi}{\alpha \beta^{2}}\left[-\alpha \beta f^{\prime} x w_{x x}+\alpha^{\prime} \beta f w_{x}+\alpha \beta^{\prime} f w_{x}+\alpha \beta^{\prime} f x w_{x x}-\frac{1}{2} \alpha \beta f^{\prime} w_{x}\right] \\
& +\frac{\epsilon^{2}}{\beta^{2}}\left[\left(-k^{2}+\frac{\beta^{\prime \prime}}{\beta}+\frac{2 \alpha^{\prime} \beta^{\prime}}{\alpha \beta}\right) x w_{x}+\frac{\left|\beta^{\prime}\right|^{2}}{\beta^{2}} x^{2} w_{x x}+\beta^{2} h^{\prime 2} w_{x x}+\frac{\alpha^{\prime \prime}}{\alpha} w\right. \\
& \left.-\frac{1}{2 \beta^{2}} V_{t t} x^{2} w-\frac{1}{2} V_{t t}\left(2 f h+h^{2}\right) w\right] \\
& -\epsilon^{2}\left[\frac{k^{2}}{\beta} h w_{x}+\frac{h^{\prime \prime}}{\beta} w_{x}+\frac{2 \beta^{\prime}}{\beta^{2}} h^{\prime} w_{x}+\frac{2 \alpha^{\prime}}{\alpha \beta} h^{\prime} w_{x}+\frac{2 \beta^{\prime}}{\beta^{2}} h^{\prime} x w_{x x}+\frac{V_{t t}}{\beta^{3}} h x w\right] \\
& -\frac{2 \epsilon^{2} \varpi}{\alpha \beta^{2}}\left[\alpha^{\prime} x w_{x}+\frac{3 \alpha \beta^{\prime}}{2 \beta} x w_{x}+\frac{\alpha \beta^{\prime}}{\beta} x^{2} w_{x x}-\alpha \beta^{2}\left(f h^{\prime}+h h^{\prime}\right) w_{x x}+\frac{1}{2} \alpha^{\prime} w\right] \\
& -\frac{2 \epsilon^{2} \varpi}{\alpha \beta^{2}}\left[-\alpha \beta h^{\prime} x w_{x x}+\alpha^{\prime} \beta h w_{x}+\alpha \beta^{\prime} h w_{x}+\alpha \beta^{\prime} h x w_{x x}-\frac{1}{2} \alpha \beta h^{\prime} w_{x}\right]+B_{2}(w) \\
\equiv & \epsilon S_{1}+\epsilon S_{2}+\epsilon^{2} S_{3}+\epsilon^{2} S_{4}+\epsilon^{2} S_{5}+\epsilon^{2} S_{6}+\epsilon^{2} S_{7}+\epsilon^{2} S_{8}+\epsilon^{2} S_{9}+B_{2}(w) .
\end{aligned}
$$

Let us observe that the quantities $S_{1}, S_{3}, S_{5}, S_{7}$ and $S_{9}$ are odd functions of $x$, while $S_{2}, S_{4}, S_{6}$ and $S_{8}$ are even functions.

For the first approximate solution $v_{1}$, the boundary errors can be formulated as follows. For $z=0$,

$$
\begin{aligned}
& \epsilon\left[b_{5} x+\beta\left(k_{1} f+f^{\prime}\right)\right] w_{x}-\epsilon \alpha^{\prime} \alpha^{-1} w \\
& +\epsilon^{2}\left[b_{1}\left(\frac{x}{\beta}+f+h\right)^{2} \beta-k\left(\frac{x}{\beta}+f+h\right)\left(\frac{\beta^{\prime}}{\beta} x-\beta f^{\prime}-\beta h^{\prime}\right)\right] w_{x} \\
& \quad-\epsilon^{2} \frac{k \alpha^{\prime}}{\alpha}\left(\frac{x}{\beta}+f+h\right) w+D_{0}^{0}(w) .
\end{aligned}
$$


Similarly, for $z=1 / \epsilon$, we have

$$
\begin{aligned}
& \epsilon\left[b_{6} x+\beta\left(k_{2} f+f^{\prime}\right)\right] w_{x}-\epsilon \alpha^{\prime} \alpha^{-1} w \\
& +\epsilon^{2}\left[b_{3}\left(\frac{x}{\beta}+f+h\right)^{2} \beta-k\left(\frac{x}{\beta}+f+h\right)\left(\frac{\beta^{\prime}}{\beta} x-\beta f^{\prime}-\beta h^{\prime}\right)\right] w_{x} \\
& -\epsilon^{2} \frac{k \alpha^{\prime}}{\alpha}\left(\frac{x}{\beta}+f+h\right) w+D_{0}^{1}(w) .
\end{aligned}
$$

\subsection{The first improvement}

We now want to construct correction terms and establish a further approximation to a real solution that eliminates the terms of order $\epsilon$ in the errors.

\subsubsection{Interior correction layers}

For any given smooth function $\phi$, it can be checked that

$$
S(w+\phi)=S(w)+L_{0}(\phi)+B_{3}(\phi)+N_{0}(\phi),
$$

where

$$
L_{0}(\phi)=\beta^{-2} \phi_{z z}+\phi_{x x}-\phi+p w^{p-1} \phi,
$$

and

$$
N_{0}(\phi)=(w+\phi)^{p}-w^{p}-p w^{p-1} \phi .
$$

By (4.2), we write

$$
\begin{aligned}
S(w+\phi)= & {\left[\epsilon\left(S_{1}+S_{2}\right)+\phi_{x x}-\phi+p w^{p-1} \phi\right]+\epsilon^{2} S_{3}+\epsilon^{2} S_{4}+\epsilon^{2} S_{5}+\epsilon^{2} S_{6} } \\
& +\epsilon^{2} S_{7}+\epsilon^{2} S_{8}+\epsilon^{2} S_{9}+B_{2}(w)+\beta^{-2} \phi_{z z}+B_{3}(\phi)+N_{0}(\phi) .
\end{aligned}
$$

We choose $\phi=\phi_{1}$ in order to eliminate the terms between brackets in the above formula. Namely, for fixed $z$, we need a solution to

$$
-\phi_{x x}+\phi-p w^{p-1} \phi=\epsilon\left(S_{1}+S_{2}\right) \quad \text { in } \mathbb{R}, \quad \phi( \pm \infty)=0 .
$$

It is well-known that this problem is solvable provided that

$$
\int_{\mathbb{R}}\left(S_{1}+S_{2}\right) w_{x} \mathrm{~d} x=0 .
$$

Furthermore, the solution is unique under the constraint

$$
\int_{\mathbb{R}} \phi w_{x} \mathrm{~d} x=0 .
$$

In fact,

$$
\begin{aligned}
\int_{\mathbb{R}}\left(S_{1}+S_{2}\right) w_{x} \mathrm{~d} x & =\int_{\mathbb{R}} S_{1} w_{x} \mathrm{~d} x \\
& =-\beta^{-1}\left[k \int_{\mathbb{R}} w_{x}^{2} \mathrm{~d} x-\frac{1}{2} \sigma^{-1} k \int_{\mathbb{R}} w^{2} \mathrm{~d} x\right] .
\end{aligned}
$$


The validity of the identity

$$
-2 \int_{\mathbb{R}} x w w_{x} \mathrm{~d} x=\int_{\mathbb{R}} w^{2} \mathrm{~d} x=2 \sigma \int_{\mathbb{R}} w_{x}^{2} \mathrm{~d} x,
$$

will precisely make the amount between brackets in (4.11) identically zero. Hence, the solution exists and has the form

$$
\phi_{1}=\epsilon\left(\phi_{11}+\phi_{12}\right)
$$

where

$$
\phi_{11}(x, z)=a_{11}(\epsilon z) w_{1}(x), \quad \phi_{12}(x, z)=[f(\epsilon z)+h(\epsilon z)] a_{12}(\epsilon z) w_{2}(x),
$$

with

$$
a_{11}(\theta)=-\frac{k(\theta)}{\beta(\theta)}, \quad a_{12}(\theta)=-\sigma^{-1} k(\theta)=-\frac{V_{t}(0, \theta)}{|\beta(\theta)|^{2}} .
$$

The function $w_{1}$ is the unique odd solution to

$$
-w_{1, x x}+w_{1}-p w^{p-1} w_{1}=w_{x}+\frac{1}{\sigma} x w, \quad \int_{\mathbb{R}} w_{1} w_{x} \mathrm{~d} x=0,
$$

and $w_{2}$ is the unique even function satisfying

$$
-w_{2, x x}+w_{2}-p w^{p-1} w_{2}=w .
$$

In fact, we can also write

$$
w_{2}=-\frac{1}{p-1} w-\frac{1}{2} x w_{x}
$$

Take

$$
v_{2}(x, z)=w(x)+\phi_{1}(x, z)
$$

as the second approximation. Substituting $\phi=\phi_{1}$ into (4.8), we get the new error

$$
\begin{aligned}
S\left(v_{2}\right)=\epsilon^{2} & \left(S_{3}+S_{4}+S_{5}+S_{6}+S_{7}+S_{8}+S_{9}\right) \\
& +B_{2}(w)+\beta^{-2} \phi_{1, z z}+B_{3}\left(\phi_{1}\right)+N_{0}\left(\phi_{1}\right) .
\end{aligned}
$$

Observe that since $\phi_{1}$ is of size $O(\epsilon)$, all terms above carry $\epsilon^{2}$ in front. We compute, for instance,

$$
B_{3}\left(\phi_{1}\right)=-\epsilon \beta^{-1}\left[k\left(\phi_{1}\right)_{x}+\sigma^{-1} k x \phi_{1}\right]-\epsilon \sigma^{-1} k(f+h) \phi_{1}+\epsilon^{3} a_{7}(\epsilon s, \epsilon z) .
$$

\subsubsection{The boundary correction layers}

In the following, we are in a position to improve the approximate solution so as to remove the boundary error terms of first order given in (4.3) and (4.4), i.e.,

$$
\epsilon b_{5} x w_{x}-\epsilon \frac{\alpha^{\prime}(0)}{\alpha(0)} w \quad \text { and } \quad \epsilon b_{6} x w_{x}-\epsilon \frac{\alpha^{\prime}(1)}{\alpha(1)} w .
$$

Note that we will deal with the boundary terms

$$
\epsilon\left(k_{1} \beta f+\beta f^{\prime}\right) w_{x} \text { and } \epsilon\left(k_{2} \beta f+\beta f^{\prime}\right) w_{x}
$$

by the standard reduction procedure. 
We first introduce a function $\mathfrak{b}(\tilde{\theta})$ satisfying

$$
\epsilon^{2} \mathfrak{b}^{\prime \prime}+\lambda_{0} \mathfrak{b}=0 \quad \text { in }(0, \ell), \quad \mathfrak{b}^{\prime}(\ell)=\mathbf{c}_{\boldsymbol{1}}, \quad \mathfrak{b}^{\prime}(0)=\mathbf{c}_{\mathbf{0}},
$$

where $\mathbf{c}_{\boldsymbol{1}}, \mathbf{c}_{\mathbf{0}}$ are constants as the following

$$
\begin{aligned}
& \mathbf{c}_{\boldsymbol{1}}=\int_{\mathbb{R}}\left[b_{6} x w_{x}-\frac{\alpha^{\prime}(1)}{\alpha(1)} w\right] Z \mathrm{~d} x, \\
& \mathbf{c}_{\mathbf{0}}=\int_{\mathbb{R}}\left[b_{5} x w_{x}-\frac{\alpha^{\prime}(0)}{\alpha(0)} w\right] Z \mathrm{~d} x .
\end{aligned}
$$

Note that the constant $\ell$ is given in (1.13), which can be also written as

$$
\ell=\int_{0}^{1} \beta(\theta) \mathrm{d} \theta
$$

where $\beta$ is the function defined in (3.22). It is easy to calculate that

$$
\mathfrak{b}(\tilde{\theta}) \equiv \epsilon A(\tilde{\theta}), \quad \forall \tilde{\theta} \in(0, \ell)
$$

where $A$ has the form

$$
A(\tilde{\theta})=\frac{\mathbf{c}_{\mathbf{0}} \cos \left(\sqrt{\lambda_{0}} \ell / \epsilon\right)-\mathbf{c}_{\boldsymbol{1}}}{\sqrt{\lambda_{0}} \sin \left(\sqrt{\lambda_{0}} \ell / \epsilon\right)} \cos \left(\frac{\sqrt{\lambda_{0}}}{\epsilon} \tilde{\theta}\right)+\frac{\mathbf{c}_{\mathbf{0}}}{\sqrt{\lambda_{0}}} \sin \left(\frac{\sqrt{\lambda_{0}}}{\epsilon} \tilde{\theta}\right) .
$$

Hence, we choose

$$
\phi_{21}(x, z)=A(\mathfrak{a}(\epsilon z)) Z(x),
$$

where

$$
\mathfrak{a}(\theta)=\int_{0}^{\theta} \beta(r) \mathrm{d} r
$$

By Corollary 2.4 in [61], we then find a unique solution $\phi_{*}$ of the following problem:

$$
\begin{array}{ll}
\Delta \phi_{*}-\phi_{*}+p w^{p-1} \phi_{*}=0 & \text { in } \hat{S}, \\
\frac{\partial \phi_{*}}{\partial \tilde{z}}=b_{6} x w_{x}-\frac{\alpha^{\prime}(1)}{\alpha(1)} w-\mathbf{c}_{1} Z & \text { on } \partial_{1} \hat{\mathcal{S}}, \\
\frac{\partial \phi_{*}}{\partial \tilde{z}}=b_{5} x w_{x}-\frac{\alpha^{\prime}(0)}{\alpha(0)} w-\mathbf{c}_{0} Z & \text { on } \partial_{0} \hat{\mathcal{S}},
\end{array}
$$

where $\hat{\mathcal{S}}, \partial_{0} \hat{\mathcal{S}}$ and $\partial_{1} \hat{\mathcal{S}}$ are defined in (3.44). Moreover, $\phi_{*}$ is even in $x$. By the diffeomophism

$$
\Upsilon:[0,1 / \epsilon] \rightarrow[0, \ell / \epsilon]
$$

in the form

$$
\Upsilon(z)=\epsilon^{-1} \int_{0}^{\epsilon z} \beta(r) \mathrm{d} r,
$$

we define

$$
\phi_{22}(x, z)=\phi_{*}(x, \Upsilon(z))
$$


It is easy to check that

$$
\begin{aligned}
\phi_{22, z}(x, z) & =\beta(\epsilon z) \phi_{*, \tilde{z}}(x, \Upsilon(z)) \\
\phi_{22, z z}(x, z) & =|\beta(\epsilon z)|^{2} \phi_{*, \tilde{z} \tilde{z}}(x, \Upsilon(z))+\epsilon \beta^{\prime}(\epsilon z) \phi_{*, \tilde{z}}(x, \Upsilon(z)) .
\end{aligned}
$$

Hence, $\phi_{22}$ satisfies the following problem:

$$
\begin{array}{rlrl}
\beta^{-2} \phi_{22, z z}+\phi_{22, x x}-\phi_{22}+p w^{p-1} \phi_{22}=\epsilon \beta^{-2} \beta^{\prime} \phi_{*, \tilde{z}}(x, \Upsilon(z)) & & \text { in } \mathcal{S}, \\
\frac{\partial \phi_{22}}{\partial z} & =\beta(1)\left[b_{6} x w_{x}-\frac{\alpha^{\prime}(1)}{\alpha(1)} w-\mathbf{c}_{1} Z\right] & & \text { on } \partial_{1} \mathcal{S}, \\
\frac{\partial \phi_{22}}{\partial z} & =\beta(0)\left[b_{5} x w_{x}-\frac{\alpha^{\prime}(0)}{\alpha(0)} w-\mathbf{c}_{0} Z\right] & & \text { on } \partial_{0} \mathcal{S},
\end{array}
$$

where $\mathcal{S}, \partial_{0} \mathcal{S}$ and $\partial_{1} \mathcal{S}$ are defined in (3.43).

We finally set the boundary correction term

$$
\phi_{2}(x, z)=\epsilon \xi(\epsilon z)\left(\phi_{21}(x, z)+\phi_{22}(x, z)\right),
$$

where

$$
\xi(\theta) \equiv \frac{\chi_{0}(\theta)}{\beta(0)}+\frac{1-\chi_{0}(\theta)}{\beta(1)},
$$

and the smooth cut-off function $\chi_{0}$ is defined by

$$
\chi_{0}(\theta)=1 \text { if }|\theta|<\frac{1}{2}, \quad \text { and } \quad \chi_{0}(\theta)=0 \text { if }|\theta|>\frac{3}{4} .
$$

Note that $\phi_{2}(x, z)$ is size of $O(\epsilon)$ under the gap condition (1.14).

Let $v_{3}(x, z)=w+\phi_{1}+\phi_{2}$ be the third approximate solution. A careful computation can indicate that the new boundary error takes the following form

$$
\begin{aligned}
& \epsilon \beta\left(k_{1} f+f^{\prime}\right) w_{x} \\
& +\epsilon^{2}\left(b_{5} x+k_{1} \beta f+\beta f^{\prime}\right)\left(a_{11} w_{1, x}+a_{12}(f+h) w_{2, x}+\beta^{-1}\left(A(0) Z_{x}+\phi_{22, x}\right)\right) \\
& -\epsilon^{2} \alpha^{\prime} \alpha^{-1}\left\{a_{11} w_{1}+a_{12}(f+h) w_{2}+\beta^{-1}\left[A(0) Z+\phi_{22}\right]\right\} \\
& -\epsilon^{2} \frac{k \alpha^{\prime}}{\alpha}\left(\frac{x}{\beta}+f+h\right)\left[w+\epsilon a_{11} w_{1}+\epsilon a_{12}(f+h) w_{2}+\epsilon \beta^{-1}\left(A(0) Z+\phi_{22}\right)\right] \\
& +\epsilon^{2}\left[b_{1}\left(\frac{x}{\beta}+f+h\right)^{2} \beta-k\left(\frac{x}{\beta}+f+h\right)\left(\frac{\beta^{\prime}}{\beta} x-\beta f^{\prime}-\beta h^{\prime}\right)\right] \\
& \times\left[w_{x}+\epsilon a_{11} w_{1, x}+\epsilon a_{12}(f+h) w_{2, x}+\epsilon \beta^{-1}\left(A(0) Z_{x}+\phi_{22, x}\right)\right] \\
& -\epsilon^{2}\left[a_{11}^{\prime} w_{1}+a_{12}^{\prime}(f+h) w_{2}+a_{12}\left(f^{\prime}+h^{\prime}\right) w_{2}\right] \\
& -\epsilon^{2} k\left(\frac{x}{\beta}+f+h\right) \times\left[\epsilon a_{11}^{\prime} w_{1}+\epsilon a_{12}^{\prime}(f+h) w_{2}+\epsilon a_{12}\left(f^{\prime}+h^{\prime}\right) w_{2}\right. \\
& \left.\quad+\beta^{-1}\left(\epsilon A^{\prime}(0) \beta(0) Z+\phi_{22, z}\right)\right]
\end{aligned}
$$




$$
\begin{aligned}
& +\epsilon^{2} b_{2}\left(\frac{x}{\beta}+f+h\right)^{2} \times\left[\epsilon^{2} a_{12}^{\prime} w_{1}+\epsilon^{2} a_{12}^{\prime}(f+h) w_{2}+\epsilon^{2} a_{12}\left(f^{\prime}+h^{\prime}\right) w_{2}\right. \\
& \left.+\epsilon \beta^{-1}\left(\epsilon A^{\prime}(0) \beta(0) Z+\phi_{22, z}\right)\right] \\
& +D_{0}^{0}\left(w+\phi_{1}+\phi_{2}\right) \\
& \text { on } \partial S_{0} .
\end{aligned}
$$

Similar estimate holds on $\partial S_{1}$.

\subsection{The second improvement}

To decompose the coupling of the parameters $f$ and $e$ on the boundary of $\mathcal{S}$ (in the sense of projection against $Z$ in $L^{2}$ ), by Lemma 2.2 in [61], we introduce a new term $\phi^{*}$ (even in $x$ ) defined by the following problem

$$
\begin{gathered}
\Delta \phi^{*}-\tilde{K} \phi^{*}+p w^{p-1} \phi^{*}=0 \quad \text { in } \hat{\mathcal{S}}, \\
\phi_{\tilde{z}}^{*}=H_{1}(x) \quad \text { on } \partial_{0} \hat{\mathcal{S}}, \quad \phi_{\tilde{z}}^{*}=H_{2}(x) \quad \text { on } \partial_{1} \hat{\mathcal{S}},
\end{gathered}
$$

where $\tilde{K}$ is a large positive constant, the functions $H_{1}(x)$ and $H_{2}(x)$ are given by the following

$$
\begin{aligned}
H_{1}(x)= & 2 b_{1}[f(0)+h(0)] x w_{x}-k(0)\left[\frac{\beta^{\prime}(0)}{\beta(0)}(f(0)+h(0))-\left(f^{\prime}(0)+h^{\prime}(0)\right)\right] x w_{x} \\
& +b_{5} x\left[a_{12}(0)(f(0)+h(0)) w_{2, x}+\beta^{-1}(0)\left(A(0) Z_{x}+\phi_{22, x}(x, 0)\right)\right] \\
& +\left[k_{1} \beta(0) f(0)+\beta(0) f^{\prime}(0)\right] a_{11}(0) w_{1, x}-k(0)(f(0)+h(0)) \frac{\alpha^{\prime}(0)}{\alpha(0)} w \\
& -\frac{\alpha^{\prime}(0)}{\alpha(0)}\left[a_{12}(0)(f(0)+h(0)) w_{2}+\beta^{-1}(0)\left(A(0) Z+\phi_{22}(x, 0)\right)\right] \\
& -\left[f^{\prime}(0)+h^{\prime}(0)\right] a_{12}(0) w_{2}-[f(0)+h(0)] a_{12}^{\prime}(0) w_{2} \\
& -k(0) \beta^{-1}(0)[f(0)+h(0)] \cdot\left[\epsilon A^{\prime}(0) \beta(0) Z+\phi_{22, z}(x, 0)\right],
\end{aligned}
$$

and

$$
\begin{aligned}
H_{2}(x)= & 2 b_{3}[f(1)+h(1)] x w_{x}-k(1)\left[\frac{\beta^{\prime}(1)}{\beta(1)}(f(1)+h(1))-\left(f^{\prime}(1)+h^{\prime}(1)\right)\right] x w_{x} \\
& +b_{6} x\left[a_{12}(1)(f(1)+h(1)) w_{2, x}+\beta^{-1}(1)\left(A(\ell) Z_{x}+\phi_{22, x}(x, 1 / \epsilon)\right)\right] \\
& +\left[k_{2} \beta(1) f(1)+\beta(1) f^{\prime}(1)\right] a_{11}(1) w_{1, x}-k(1)(f(1)+h(1)) \frac{\alpha^{\prime}(1)}{\alpha(1)} w \\
& -\frac{\alpha^{\prime}(1)}{\alpha(1)}\left[a_{12}(1)(f(1)+h(1)) w_{2}+\beta^{-1}(1)\left(A(\ell) Z+\phi_{22}(x, 1 / \epsilon)\right)\right] \\
& -\left[f^{\prime}(1)+h^{\prime}(1)\right] a_{12}(1) w_{2}-[f(1)+h(1)] a_{12}^{\prime}(1) w_{2} \\
& -k(1) \beta^{-1}(1)[f(1)+h(1)] \cdot\left[\epsilon A^{\prime}(\ell) \beta(1) Z+\phi_{22, z}(x, 1 / \epsilon)\right] .
\end{aligned}
$$

We define a boundary correction term again

$$
\phi_{3}(x, z)=\epsilon^{2} \xi(\epsilon z) \phi^{*}(x, \Upsilon(z)) \equiv \epsilon^{2} \hat{\phi}(x, z) .
$$

Note that $\phi_{3}$ is an exponential decaying function which is of order $\epsilon^{2}$ and even in the variable $x$. 
To deal with the resonance phenomena and improve the approximation for a solution still keeping the term of $\epsilon^{2}$, we need to introduce a new parameter $e$ in additional to $f$ and $h$, and define the fourth approximate solution to the problem near $\Gamma_{\epsilon}$ as

$$
v_{4}(x, z)=w+\phi_{1}+\phi_{2}+\epsilon e(\epsilon z) Z(x)+\phi_{3} .
$$

\subsection{The third improvement}

We want to construct a further approximation to a solution that eliminates the term of $h, h^{\prime}$ and the even terms in the error

$$
S\left(w+\phi_{1}+\phi_{2}+\epsilon e Z+\phi_{3}\right) .
$$

This can be fulfilled by adding a term $\tilde{\phi}$ and then considering the following term

$$
\begin{aligned}
& S\left(w+\phi_{1}+\phi_{2}+\epsilon e Z+\phi_{3}+\tilde{\phi}\right) \\
&= S(w)+L_{0}\left(\phi_{1}+\phi_{2}+\epsilon e Z+\phi_{3}+\tilde{\phi}\right)+B_{3}\left(\phi_{1}\right)+B_{3}\left(\phi_{2}\right) \\
&+B_{3}(\epsilon e Z)+B_{3}\left(\phi_{3}\right)+B_{3}(\tilde{\phi})+N_{0}\left(\phi_{1}+\phi_{2}+\epsilon e Z+\phi_{3}+\tilde{\phi}\right) \\
&= S(w)+\epsilon\left(\epsilon^{2} \beta^{-2} e^{\prime \prime} Z+\lambda_{0} e Z\right)+L_{0}\left(\phi_{1}\right)+L_{0}\left(\phi_{2}\right)+L_{0}\left(\phi_{3}\right)+L_{0}(\tilde{\phi}) \\
&+B_{3}\left(\phi_{1}\right)+B_{3}\left(\phi_{2}\right)+B_{3}(\epsilon e Z)+B_{3}\left(\phi_{3}\right)+B_{3}(\tilde{\phi})+N_{0}\left(\phi_{1}+\phi_{2}+\epsilon e Z+\phi_{3}+\tilde{\phi}\right) .
\end{aligned}
$$

The details will be given in the sequel.

\subsubsection{Rearrangements of the error components}

The first objective of this part is to compute the terms in (4.36). It is easy to compute that

$$
L_{0}\left(\phi_{1}\right)=\beta^{-2} \phi_{1, z z}+\phi_{1, x x}-\phi_{1}+p w^{p-1} \phi_{1}=\beta^{-2} \phi_{1, z z}-\epsilon\left(S_{1}+S_{2}\right) .
$$

Recall the expression of $\phi_{2}$ and $A(\tilde{\theta})$ defined in (4.30) and (4.24). By using the equation of $\phi_{22}$ in (4.29) and the equation of $Z$ in (1.11), we get

$$
\begin{aligned}
L_{0}\left(\phi_{2}\right) & =\beta^{-2} \phi_{2, z z}+\phi_{2, x x}-\phi_{2}+p w^{p-1} \phi_{2} \\
& =M_{11}(x, z)+M_{12}(x, z),
\end{aligned}
$$

where

$$
M_{11}(x, z)=\frac{2 \epsilon^{2}}{\beta^{2}} \xi^{\prime}(\epsilon z)\left[\epsilon A^{\prime}(\mathfrak{a}(\epsilon z)) \beta Z+\phi_{22, z}\right]+\frac{\epsilon^{2} \beta^{\prime}}{\beta^{2}} \xi(\epsilon z)\left[\epsilon A^{\prime}(\mathfrak{a}(\epsilon z)) Z+\phi_{*, \tilde{z}}\right],
$$

and

$$
M_{12}(x, z)=\frac{\epsilon^{3}}{\beta^{2}} \xi^{\prime \prime}(\epsilon z)\left[A(\mathfrak{a}(\epsilon z)) Z+\phi_{22}\right]
$$

According to the fact that $\phi^{*}$ satisfies

$$
\Delta \phi^{*}-\tilde{K} \phi^{*}+p w^{p-1} \phi^{*}=0 \text { in } \hat{\mathcal{S}},
$$


and

$$
\phi_{3}=\epsilon^{2} \hat{\phi}=\epsilon^{2} \xi(\epsilon z) \phi^{*}
$$

it follows that

$$
\begin{aligned}
L_{0}\left(\phi_{3}\right) & =\epsilon^{2}\left[\beta^{-2} \hat{\phi}_{z z}+\hat{\phi}_{x x}-\hat{\phi}+p w^{p-1} \hat{\phi}\right] \\
& =\epsilon^{2}(\tilde{K}-1) \hat{\phi}+\epsilon^{2}\left[\beta^{-2} \hat{\phi}_{z z}+\hat{\phi}_{x x}-\tilde{K} \hat{\phi}+p w^{p-1} \hat{\phi}\right] \\
& =M_{21}(x, z)+M_{22}(x, z),
\end{aligned}
$$

where

$$
M_{21}(x, z)=\epsilon^{2}(\tilde{K}-1) \hat{\phi},
$$

and

$$
M_{22}(x, z)=\frac{\epsilon^{4}}{\beta^{2}} \xi^{\prime \prime}(\epsilon z) \phi^{*}(x, \Upsilon(z))+\frac{2 \epsilon^{3}}{\beta} \xi^{\prime}(\epsilon z) \phi_{\tilde{z}}^{*}(x, \Upsilon(z))+\frac{\epsilon^{3} \beta^{\prime}}{\beta^{2}} \xi(\epsilon z) \phi_{\tilde{z}}^{*}(x, \Upsilon(z))
$$

Recall the expression of $B_{3}\left(\phi_{1}\right)$ in (4.20), we obtain that

$$
\begin{aligned}
B_{3}\left(\phi_{1}\right) & =-\epsilon \beta^{-1}\left[k\left(\phi_{1}\right)_{x}+\sigma^{-1} k x \phi_{1}\right]-\epsilon \sigma^{-1} k(f+h) \phi_{1}+\epsilon^{3} a_{7}(\epsilon s, \epsilon z) \\
& =M_{31}(x, z)+M_{32}(x, z),
\end{aligned}
$$

where

$$
M_{31}(x, z)=\frac{\epsilon^{2} k^{2}}{\beta \sigma}\left[\frac{\sigma}{\beta} w_{1, x}+h w_{2, x}+\frac{1}{\beta} x w_{1}+\frac{1}{\sigma} h x w_{2}+h w_{1}+\frac{\beta}{\sigma} h^{2} w_{2}+\frac{2 \beta}{\sigma} f h w_{2}\right],
$$

and

$$
M_{32}(x, z)=\frac{\epsilon^{2} k^{2}}{\beta \sigma}\left[f w_{2, x}+\sigma^{-1} f x w_{2}+f w_{1}+\sigma^{-1} \beta f^{2} w_{2}\right]+\epsilon^{3} a_{7}(\epsilon s, \epsilon z) .
$$

What's more, we can decompose $B_{3}\left(\phi_{2}\right)$ as following

$$
\begin{aligned}
B_{3}\left(\phi_{2}\right)= & -\epsilon \beta^{-1} k \phi_{2, x}+2 \epsilon \beta^{-2}\left(\frac{\beta^{\prime}}{\beta} x-\beta f^{\prime}-\beta h^{\prime}\right) \phi_{2, x z}+\frac{2 \epsilon \alpha^{\prime}}{\alpha \beta^{2}} \phi_{2, z}-\frac{2 \epsilon \varpi}{\beta}\left(\frac{x}{\beta}+f+h\right) \phi_{2, x z} \\
& -\frac{\epsilon \varpi}{\beta^{2}} \phi_{2, z}-\epsilon \sigma^{-1} k\left(\frac{x}{\beta}+f+h\right) \phi_{2}+\epsilon^{3} a_{8}(\epsilon s, \epsilon z) \\
= & M_{41}(x, z)+M_{42}(x, z),
\end{aligned}
$$

where

$$
\begin{aligned}
M_{41}(x, z)= & -\frac{\epsilon^{2}}{\beta} k \xi(\epsilon z)\left[A(\mathfrak{a}(\epsilon z)) Z_{x}+\phi_{22, x}\right]+\frac{2 \epsilon^{2}}{\beta^{2}}\left(\frac{\beta^{\prime}}{\beta} x-\beta h^{\prime}\right) \xi(\epsilon z)\left[\epsilon A^{\prime}(\mathfrak{a}(\epsilon z)) \beta Z_{x}+\phi_{22, x z}\right] \\
& +\frac{2 \epsilon^{2} \alpha^{\prime}}{\alpha \beta^{2}} \xi(\epsilon z)\left[\epsilon A^{\prime}(\mathfrak{a}(\epsilon z)) \beta Z+\phi_{22, z}\right]-\frac{2 \epsilon^{2} \varpi}{\beta}\left(\frac{x}{\beta}+h\right) \xi(\epsilon z)\left[\epsilon A^{\prime}(\mathfrak{a}(\epsilon z)) \beta Z_{x}+\phi_{22, x z}\right] \\
& -\frac{\epsilon^{2} \varpi}{\beta^{2}} \xi(\epsilon z)\left[\epsilon A^{\prime}(\mathfrak{a}(\epsilon z)) \beta Z+\phi_{22, z}\right]-\epsilon^{2} \sigma^{-1} k\left(\frac{x}{\beta}+f+h\right) \xi(\epsilon z)\left[A(\mathfrak{a}(\epsilon z)) Z+\phi_{22}\right],
\end{aligned}
$$

and

$$
M_{42}(x, z)=-\frac{2 \epsilon^{2}}{\beta}\left(f^{\prime}+\varpi f\right) \xi(\epsilon z)\left[\epsilon A^{\prime}(\mathfrak{a}(\epsilon z)) \beta Z_{x}+\phi_{22, x z}\right]+\epsilon^{3} a_{8}(\epsilon s, \epsilon z) .
$$


The computation for next term is the following:

$$
\begin{aligned}
B_{3}(\epsilon e Z) & =-\epsilon^{2} \beta^{-1} k e Z_{x}-\epsilon^{2} \sigma^{-1} k\left(\frac{x}{\beta}+f+h\right) e Z+\epsilon^{3} a_{9}(\epsilon s, \epsilon z) \\
& =M_{51}(x, z)+M_{52}(x, z),
\end{aligned}
$$

where

$$
M_{51}(x, z)=-\epsilon^{2} \sigma^{-1} k(f+h) e Z,
$$

and

$$
M_{52}(x, z)=-\epsilon^{2} \beta^{-1} k e Z_{x}-\epsilon^{2} \sigma^{-1} \beta^{-1} k x e Z+\epsilon^{3} a_{9}(\epsilon s, \epsilon z) .
$$

The main order of the nonlinear term

$$
\begin{aligned}
N_{0}\left(\phi_{1}+\phi_{2}+\epsilon e Z+\phi_{3}+\tilde{\phi}\right)= & \left(w+\phi_{1}+\phi_{2}+\epsilon e Z+\phi_{3}+\tilde{\phi}\right)^{p}-w^{p} \\
& -p w^{p-1}\left(\phi_{1}+\phi_{2}+\epsilon e Z+\phi_{3}+\tilde{\phi}\right),
\end{aligned}
$$

is in the following

$$
\frac{p(p-1)}{2} w^{p-2}\left(\phi_{1}+\phi_{2}+\epsilon e Z+\phi_{3}+\tilde{\phi}\right)^{2},
$$

which can be decomposed as following

$$
\begin{aligned}
& \frac{p(p-1)}{2} w^{p-2}\left(\phi_{1}+\phi_{2}+\epsilon e Z+\phi_{3}+\tilde{\phi}\right)^{2} \\
& =\frac{p(p-1)}{2} w^{p-2}\left[\phi_{1}^{2}+\phi_{2}^{2}+\epsilon^{2} e^{2} Z^{2}+\epsilon^{4} \hat{\phi}^{2}+\tilde{\phi}^{2}+2 \phi_{1} \phi_{2}+2 \epsilon \phi_{1} e Z+2 \epsilon^{2} \phi_{1} \hat{\phi}\right. \\
& \left.+2 \phi_{1} \tilde{\phi}+2 \epsilon \phi_{2} e Z+2 \epsilon^{2} \phi_{2} \hat{\phi}+2 \phi_{2} \tilde{\phi}+2 \epsilon^{3} e Z \hat{\phi}+2 \epsilon e Z \tilde{\phi}+2 \epsilon^{2} \hat{\phi} \tilde{\phi}\right] \\
& =\epsilon^{2} \frac{p(p-1)}{2} w^{p-2}\left[a_{11}^{2} w_{1}^{2}+2 a_{11} a_{12} h w_{1} w_{2}+a_{12}^{2}\left(2 f h+h^{2}\right) w_{2}^{2}+\xi(\epsilon z)^{2}\left(A\left(\mathfrak{a}(\epsilon z) Z+\phi_{22}\right)^{2}\right.\right. \\
& +e^{2} Z^{2}+2 a_{11} w_{1} \xi(\epsilon z)\left(A\left(\mathfrak{a}(\epsilon z) Z+\phi_{22}\right)+2 a_{12}(f+h) w_{2} e Z\right. \\
& +2 a_{12}(f+h) w_{2} \xi(\epsilon z)\left(A\left(\mathfrak{a}(\epsilon z) Z+\phi_{22}\right)+2 \xi(\epsilon z)\left(A\left(\mathfrak{a}(\epsilon z) Z+\phi_{22}\right) e Z\right]\right. \\
& +\frac{p(p-1)}{2} w^{p-2}\left[2 \epsilon^{2} a_{11} a_{12} w_{1} w_{2} f+\epsilon^{2} a_{12}^{2} w_{2}^{2} f^{2}+2 \epsilon^{2} a_{11} w_{1} e Z+\epsilon^{4} \hat{\phi}^{2}+\tilde{\phi}^{2}+2 \epsilon^{2} \phi_{1} \hat{\phi}\right. \\
& \left.+2 \phi_{1} \tilde{\phi}+2 \epsilon^{2} \phi_{2} \hat{\phi}+2 \phi_{2} \tilde{\phi}+2 \epsilon^{3} e Z \hat{\phi}+2 \epsilon e Z \tilde{\phi}+2 \epsilon^{2} \hat{\phi} \tilde{\phi}\right] \\
& \equiv M_{61}(x, z)+\tilde{M}_{62}(x, z)
\end{aligned}
$$

For the convenience of notation, we also denote

$$
M_{62}(x, z)=N_{0}\left(\phi_{1}+\phi_{2}+\epsilon e Z+\phi_{3}+\tilde{\phi}\right)-M_{61}(x, z) .
$$


Whence, according to the above rearrangements, we rewrite the expression (4.36) in terms of

$$
\begin{aligned}
& S\left(w+\phi_{1}+\phi_{2}+\epsilon e Z+\phi_{3}+\tilde{\phi}\right) \\
& =\left[\epsilon^{2} S_{6}+\epsilon^{2} S_{7}+\epsilon^{2} S_{8}+\epsilon^{2} S_{9}+M_{11}(x, z)+M_{21}(x, z)+M_{31}(x, z)\right. \\
& \left.\quad \quad+M_{41}(x, z)+M_{51}(x, z)+M_{61}(x, z)+\tilde{\phi}_{x x}-\tilde{\phi}+p w^{p-1} \tilde{\phi}\right] \\
& \quad+\epsilon^{2} S_{3}+\epsilon^{2} S_{4}+\epsilon^{2} S_{5}+B_{2}(w)+\beta^{-2} \phi_{1, z z}+\beta^{-2} \tilde{\phi}_{z z}+\epsilon\left(\epsilon^{2} \beta^{-2} e^{\prime \prime} Z+\lambda_{0} e Z\right) \\
& \quad+M_{12}(x, z)+M_{22}(x, z)+M_{32}(x, z)+M_{42}(x, z)+M_{52}(x, z) \\
& \quad+M_{62}(x, z)+B_{3}\left(\phi_{3}\right)+B_{3}(\tilde{\phi}) .
\end{aligned}
$$

\subsubsection{Finding new correction terms and defining the basic approximation}

We now choose $\tilde{\phi}=\phi_{4}$ in order to eliminate the terms between the first two brackets in (4.51).

Namely, for fixed $z$, we need a solution to the problem

$$
\begin{aligned}
-\tilde{\phi}_{x x}+\tilde{\phi}-p w^{p-1} \tilde{\phi}= & \epsilon^{2} S_{6}+\epsilon^{2} S_{7}+\epsilon^{2} S_{8}+\epsilon^{2} S_{9}+M_{11}(x, z)+M_{21}(x, z) \\
& +M_{31}(x, z)+M_{41}(x, z)+M_{51}(x, z)+M_{61}(x, z), \quad \forall x \in \mathbb{R} .
\end{aligned}
$$

It is well-known that this problem is solvable provided that

$$
\begin{aligned}
\int_{\mathbb{R}}\left[\epsilon^{2} S_{6}\right. & +\epsilon^{2} S_{7}+\epsilon^{2} S_{8}+\epsilon^{2} S_{9}+M_{11}(x, z)+M_{21}(x, z) \\
& \left.+M_{31}(x, z)+M_{41}(x, z)+M_{51}(x, z)+M_{61}(x, z)\right] w_{x} \mathrm{~d} x=0 .
\end{aligned}
$$

The computation of (4.53) can be showed as follows. Since $S_{6}, S_{8}, M_{11}(x, z), M_{21}(x, z)$, $M_{51}(x, z)$ are even functions, integration against $w_{x}$ therefore just vanish. This gives that

$$
\begin{aligned}
& \int_{\mathbb{R}}\left[\epsilon^{2} S_{6}+\epsilon^{2} S_{7}+\epsilon^{2} S_{8}+\epsilon^{2} S_{9}+M_{11}(x, z)+M_{21}(x, z)\right. \\
& \left.\quad+M_{31}(x, z)+M_{41}(x, z)+M_{51}(x, z)+M_{61}(x, z)\right] w_{x} \mathrm{~d} x \\
& =\int_{\mathbb{R}}\left[\epsilon^{2} S_{7}+\epsilon^{2} S_{9}+M_{31}(x, z)+M_{41}(x, z)+M_{61}(x, z)\right] w_{x} \mathrm{~d} x \\
& \equiv J_{1}+J_{2}+J_{3}+J_{4}+J_{5} .
\end{aligned}
$$

Recalling the expression of $S_{7}$ in (4.2), direct computation leads to

$$
\begin{aligned}
J_{1}= & -\epsilon^{2} \beta^{-1} h^{\prime \prime} \int_{\mathbb{R}} w_{x}^{2} \mathrm{~d} x-2 \epsilon^{2} \beta^{-2} \beta^{\prime} h^{\prime} \int_{\mathbb{R}}\left(w_{x}^{2}+x w_{x} w_{x x}\right) \mathrm{d} x \\
& -2 \epsilon^{2} \alpha^{-1} \beta^{-1} \alpha^{\prime} h^{\prime} \int_{\mathbb{R}} w_{x}^{2} \mathrm{~d} x-\epsilon^{2} h\left(\beta^{-1} k^{2} \int_{\mathbb{R}} w_{x}^{2} \mathrm{~d} x+\beta^{-3} V_{t t} \int_{\mathbb{R}} x w w_{x} \mathrm{~d} x\right) \\
= & -\epsilon^{2} \varrho_{1} \beta^{-1} h^{\prime \prime}-\epsilon^{2} \varrho_{1} \beta^{-1}\left(\beta^{-1} \beta^{\prime}+2 \alpha^{-1} \alpha^{\prime}\right) h^{\prime}-\epsilon^{2} \varrho_{1} \beta^{-1}\left(k^{2}-\sigma \beta^{-2} V_{t t}\right) h,
\end{aligned}
$$

where we have used (4.12) and the following relation

$$
\varrho_{1} \equiv \int_{\mathbb{R}} w_{x}^{2} \mathrm{~d} x=-2 \int_{\mathbb{R}} x w_{x} w_{x x} \mathrm{~d} x .
$$


According to the definition of $S_{9}$, it follows that

$$
\begin{aligned}
J_{2}= & 2 \epsilon^{2} \beta^{-1} h^{\prime} \varpi \int_{\mathbb{R}}\left(x w_{x x} w_{x}+\frac{1}{2} w_{x}^{2}\right) \mathrm{d} x-2 \epsilon^{2} \alpha^{-1} \alpha^{\prime} \beta^{-1} h \varpi \int_{\mathbb{R}} w_{x}^{2} \mathrm{~d} x \\
& \quad-2 \epsilon^{2} \beta^{\prime} \beta^{-2} h \varpi \int_{\mathbb{R}}\left(w_{x}^{2}+x w_{x x} w_{x}\right) \mathrm{d} x \\
= & -\epsilon^{2} \varrho_{1} \beta^{-1}\left[\varpi \beta^{\prime} \beta^{-1}+2 \varpi \alpha^{-1} \alpha^{\prime}\right] h .
\end{aligned}
$$

Since $w_{1}$ is an odd function and $w_{2}$ is a even function, we obtain

$$
\begin{aligned}
J_{3}=\int_{\mathbb{R}} M_{31}(x, z) w_{x} \mathrm{~d} x & =\epsilon^{2} \beta^{-1} \sigma^{-1} k^{2} \int_{\mathbb{R}}\left[h w_{2, x}+\sigma^{-1} h x w_{2}+h w_{1}\right] w_{x} \mathrm{~d} x \\
& =\epsilon^{2} \beta^{-1} \sigma^{-1} k^{2} h \int_{\mathbb{R}}\left[w_{2, x} w_{x}+\frac{1}{\sigma} x w_{2} w_{x}+w_{1} w_{x}\right] \mathrm{d} x .
\end{aligned}
$$

By the definition of $M_{41}(x, z)$, we get

$$
\begin{aligned}
J_{4} & =\int_{\mathbb{R}} M_{41}(x, z) w_{x} \mathrm{~d} x \\
& =-\epsilon^{2} \varrho_{1} \beta^{-1} \alpha_{1}(z) h^{\prime}-\epsilon^{2} \varrho_{1} \beta^{-1} \alpha_{2}(z) h+\epsilon^{2} \varrho_{1} \beta^{-1}\left[G_{1}(z)+G_{2}(z)\right],
\end{aligned}
$$

where

$$
\begin{gathered}
\alpha_{1}(z)=2 \varrho_{1}^{-1} \xi(\epsilon z) \int_{\mathbb{R}}\left[\epsilon A^{\prime}(\mathfrak{a}(\epsilon z)) \beta(\epsilon z) Z_{x}+\phi_{22, x z}(x, z)\right] w_{x} \mathrm{~d} x, \\
\alpha_{2}(z)=\varpi(\epsilon z) \alpha_{1}(z), \\
G_{1}(z)=-\frac{k(\epsilon z)}{\varrho_{1}} \xi(\epsilon z) \int_{\mathbb{R}}\left[A(\mathfrak{a}(\epsilon z)) Z_{x}+\phi_{22, x}(x, z)\right] w_{x} \mathrm{~d} x, \\
G_{2}(z)=-\frac{k(\epsilon z)}{\sigma \varrho_{1}} \xi(\epsilon z) \int_{\mathbb{R}}\left[A(\mathfrak{a}(\epsilon z)) Z+\phi_{22}(x, z)\right] x w_{x} \mathrm{~d} x .
\end{gathered}
$$

Since $w_{1}$ and $w_{x}$ are odd functions, while $w_{2}, \phi_{21}$ and $\phi_{22}$ are even functions, so we obtain that

$$
\begin{aligned}
J_{5}= & \epsilon^{2} p(p-1) \int_{\mathbb{R}}\left[a_{11} a_{12} h w_{1} w_{2}+a_{11} w_{1} \xi(\epsilon z)\left(\phi_{21}+\phi_{22}\right)\right] w^{p-2} w_{x} \mathrm{~d} x \\
= & \epsilon^{2} a_{11} a_{12} h \int_{\mathbb{R}} p(p-1) w^{p-2} w_{1} w_{2} w_{x} \mathrm{~d} x \\
& \quad+\epsilon^{2} a_{11} \xi(\epsilon z) \int_{\mathbb{R}} p(p-1) w^{p-2} w_{1}\left(\phi_{21}+\phi_{22}\right) w_{x} \mathrm{~d} x \\
\equiv & \epsilon^{2} \beta^{-1} \sigma^{-1} k^{2} h \int_{\mathbb{R}} p(p-1) w^{p-2} w_{1} w_{2} w_{x} \mathrm{~d} x+\epsilon^{2} \varrho_{1} \beta^{-1} G_{3}(z),
\end{aligned}
$$

where

$$
G_{3}(z)=a_{11} \beta \varrho_{1}^{-1} \xi(\epsilon z) p(p-1) \int_{\mathbb{R}} w^{p-2} w_{1}\left[A(\mathfrak{a}(\epsilon z)) Z+\phi_{22}(x, z)\right] w_{x} \mathrm{~d} x .
$$

By differentiating the equation (4.17) and using equation (4.16), we obtain

$$
\int_{\mathbb{R}} p(p-1) w^{p-2} w_{x} w_{1} w_{2} \mathrm{~d} x=-\int_{\mathbb{R}} w_{x} w_{1} \mathrm{~d} x+\int_{\mathbb{R}}\left(w_{x}+\frac{1}{\sigma} x w\right) w_{2, x} \mathrm{~d} x .
$$


Adding (4.57), (4.63) and using (4.65), we have

$$
\begin{aligned}
J_{3}+J_{5}= & \epsilon^{2} \beta^{-1} \sigma^{-1} k^{2} h \int_{\mathbb{R}}\left[p(p-1) w^{p-2} w_{1} w_{2} w_{x}+w_{2, x} w_{x}+\frac{1}{\sigma} x w_{2} w_{x}\right. \\
& \left.+w_{1} w_{x}\right] \mathrm{d} x+\epsilon^{2} \varrho_{1} \beta^{-1} G_{3}(z) \\
& =\epsilon^{2} \beta^{-1} \sigma^{-1} k^{2} h \int_{\mathbb{R}}\left[2 w_{2, x} w_{x}+\sigma^{-1} x\left(w_{2} w\right)_{x}\right] \mathrm{d} x+\epsilon^{2} \varrho_{1} \beta^{-1} G_{3}(z) \\
& =-\epsilon^{2} \varrho_{1} \beta^{-1} \sigma^{-1} k^{2} h+\epsilon^{2} \varrho_{1} \beta^{-1} G_{3}(z),
\end{aligned}
$$

where we have used (2.9) and the following integral identities

$$
2 \int_{\mathbb{R}} w_{2, x} w_{x} \mathrm{~d} x=-\left(\frac{2}{p-1}+\frac{1}{2}\right) \int_{\mathbb{R}} w_{x}^{2} \mathrm{~d} x, \quad \sigma^{-1} \int_{\mathbb{R}} w_{2} w \mathrm{~d} x=\left(\frac{1}{2}-\frac{2}{p-1}\right) \int_{\mathbb{R}} w_{x}^{2} \mathrm{~d} x .
$$

Consequently, we infer that

$$
\begin{aligned}
\int_{\mathbb{R}}\left[\epsilon^{2} S_{6}\right. & +\epsilon^{2} S_{7}+\epsilon^{2} S_{8}+\epsilon^{2} S_{9}+M_{11}(x, z)+M_{21}(x, z) \\
& \left.+M_{31}(x, z)+M_{41}(x, z)+M_{51}(x, z)+M_{61}(x, z)\right] w_{x} \mathrm{~d} x \\
=-\epsilon^{2} \varrho_{1} \beta^{-1}\left[h^{\prime \prime}+\left(\hbar_{1}(\epsilon z)+\alpha_{1}(z)\right) h^{\prime}+\left(\hbar_{2}(\epsilon z)+\alpha_{2}(z)\right) h\right] & \\
& +\epsilon^{2} \varrho_{1} \beta^{-1}\left[G_{1}(z)+G_{2}(z)+G_{3}(z)\right],
\end{aligned}
$$

where functions $\hbar_{1}$ and $\hbar_{2}$ are given by

$$
\hbar_{1}(\theta)=\beta^{-1} \beta^{\prime}+2 \alpha^{-1} \alpha^{\prime}=\sigma V^{-1} V_{\theta},
$$

and

$$
\begin{aligned}
\hbar_{2}(\theta) & =-\sigma V^{-1} V_{t t}+\left(\sigma^{-1}+1\right) k^{2}+\varpi\left(\beta^{-1} \beta^{\prime}+2 \alpha^{-1} \alpha^{\prime}\right) \\
& =\sigma V^{-1} V_{\theta} \Theta_{t t}-\sigma V^{-1} V_{t t}+\left(\sigma^{-1}+1\right) k^{2} .
\end{aligned}
$$

The above computation give that the validity of (4.53) holds if the following problem

$$
\begin{gathered}
h^{\prime \prime}+\left(\hbar_{1}(\epsilon z)+\alpha_{1}(z)\right) h^{\prime}+\left(\hbar_{2}(\epsilon z)+\alpha_{2}(z)\right) h=G_{1}(z)+G_{2}(z)+G_{3}(z), \\
h^{\prime}(1)+k_{2} h(1)=0, \quad h^{\prime}(0)+k_{1} h(0)=0,
\end{gathered}
$$

has a solution. In fact, the solvability of problem (4.69) will be established in Lemma 7.1. Moreover, $h$ has the following estimate

$$
\|h\|_{*} \leq C \epsilon^{\frac{1}{2}}
$$

where the norm $\|\cdot\|_{*}$ is given in $(3.23)$.

We can now find a function defined by $\tilde{\phi}=\phi_{4}(x, z)$, such that the terms between brackets in (4.51) disappear. More precisely, the solution has the form

$$
\phi_{4}(x, z)=\epsilon^{2} \phi_{41}(x, \epsilon z)+\epsilon^{2} \phi_{42}(x, \epsilon z),
$$


where $\phi_{41}(x, \theta)$ and $\phi_{42}(x, \theta)$ only depend on $f(\theta), e(\theta)$, but their derivatives. The function $\epsilon^{2} \phi_{41}(x, \theta)$ satisfies the following equation, for fixed $\theta=\epsilon z$

$$
\begin{aligned}
&-\phi_{x x}+\phi-p w^{p-1} \phi \\
&=\epsilon^{2} S_{6}+\epsilon^{2} S_{8}+M_{11}+M_{21}+M_{51}+\frac{\epsilon^{2} k^{2}}{\beta \sigma}\left[\frac{\sigma}{\beta} w_{1, x}+\frac{1}{\beta} x w_{1}+\frac{\beta}{\sigma} h^{2} w_{2}+\frac{2 \beta}{\sigma} f h w_{2}\right] \\
&+\frac{2 \epsilon^{2}}{\beta^{2}} \xi\left(\frac{\beta^{\prime}}{\beta}-\varpi\right)\left[\epsilon A^{\prime}(\mathfrak{a}) \beta x Z_{x}+x \phi_{22, x z}\right]-\epsilon^{2} \sigma^{-1} k(f+h) \xi\left[A(\mathfrak{a}) Z+\phi_{22}\right] \\
&+\frac{\epsilon^{2}}{\beta^{2}} \xi\left(\frac{2 \alpha^{\prime}}{\alpha}-\varpi\right)\left[\epsilon A^{\prime}(\mathfrak{a}) \beta Z+\phi_{22, z}\right] \\
&+\epsilon^{2} \frac{p(p-1)}{2} w^{p-2}\left[a_{11}^{2} w_{1}^{2}+a_{12}^{2}\left(2 f h+h^{2}\right) w_{2}^{2}+e^{2} Z^{2}+\xi^{2}\left(A(\mathfrak{a}) Z+\phi_{22}\right)^{2}\right. \\
&+2 a_{12}(f+h) w_{2} \xi\left(A(\mathfrak{a}) Z+\phi_{22}\right) \\
&\left.+2 a_{12}(f+h) w_{2} e Z+2 \xi\left(A(\mathfrak{a}) Z+\phi_{22}\right) e Z\right]
\end{aligned}
$$

and $\phi_{42}(x, \theta)$ satisfies

$$
\begin{aligned}
- & \phi_{x x}+\phi-p w^{p-1} \phi \\
= & S_{7}+S_{9}+\frac{k^{2}}{\beta \sigma}\left[h w_{2, x}+\sigma^{-1} h x w_{2}+h w_{1}\right]-\frac{k}{\beta} \xi\left[A(\mathfrak{a}) Z_{x}+\phi_{22, x}\right] \\
& -\frac{2}{\beta} h^{\prime} \xi\left[\epsilon A^{\prime}(\mathfrak{a}) \beta Z_{x}+\phi_{22, x z}\right]-\frac{2 \varpi}{\beta} h \xi\left[\epsilon A^{\prime}(\mathfrak{a}) \beta Z_{x}+\phi_{22, x z}\right] \\
& -\sigma^{-1} \beta^{-1} k x \xi\left[A(\mathfrak{a}) Z+\phi_{22}\right]+p(p-1) w^{p-2}\left[a_{11} a_{12} h w_{1} w_{2}+a_{11} w_{1} \xi\left(A(\mathfrak{a}) Z+\phi_{22}\right)\right] .
\end{aligned}
$$

Finally, our basic approximate solution $v$ in (3.45) to the problem near the curve $\Gamma_{\epsilon}$ is

$$
v_{5}(x, z)=w(x)+\phi_{1}(x, z)+\phi_{2}(x, z)+\epsilon e(\epsilon z) Z(x)+\phi_{3}(x, z)+\phi_{4}(x, z) .
$$

\subsection{The global approximate solution and the errors}

Recall the relation (1.16), the coordinates $(t, \theta)$ in $(2.4),(s, z)$ in $(3.13)$ and $(x, z)$ in $(3.45)$ and also the local approximate solution $v_{5}(x, z)$ in (4.70), which is constructed near the curve $\Gamma_{\epsilon}$ in the coordinates $(x, z)$. By the relation in (3.45), we then make an extension and simply define the approximate solution to (1.17) in the form

$$
\mathbf{W}(\tilde{y})=\eta_{3 \delta}^{\epsilon}(s) \alpha(\epsilon z) v_{5}(\beta(\epsilon z)(s-\epsilon f(\epsilon z)-\epsilon h(\epsilon z)), z) .
$$

Note that, in the coordinates $\left(\tilde{y}_{1}, \tilde{y}_{2}\right)$ introduced in (1.16), $\mathbf{W}$ is a function defined on $\Omega_{\epsilon}$ which is extended globally as 0 beyond the $6 \delta / \epsilon$-neighborhood of $\Gamma_{\epsilon}$ and also satisfies (3.10).

Recalling (3.46), (3.48) and (3.49), the local forms of the error terms can now be formulated. The interior error $\mathcal{E}$ can be arranged as follows

$$
\begin{aligned}
\mathcal{E} \equiv & S\left(v_{5}\right)=S\left(w+\phi_{1}+\phi_{2}+\epsilon e Z+\phi_{3}+\phi_{4}\right) \\
= & \epsilon^{2} S_{3}+\epsilon^{2} S_{4}+\epsilon^{2} S_{5}+B_{2}(w)+\epsilon^{3} \beta^{-2} e^{\prime \prime} Z+\epsilon \lambda_{0} e Z \\
& +\beta^{-2} \phi_{1, z z}+\beta^{-2} \phi_{4, z z}+M_{12}(x, z)+M_{22}(x, z)+M_{32}(x, z) \\
& +M_{42}(x, z)+M_{52}(x, z)+M_{62}(x, z)+B_{3}\left(\phi_{3}\right)+B_{3}\left(\phi_{4}\right),
\end{aligned}
$$


where we have used (4.51) and the equation of $\phi_{4}$ in (4.52). The boundary error term $g_{0}$ has the form

$$
\begin{aligned}
g_{0}(x)= & -\epsilon \beta\left(k_{1} f+f^{\prime}\right) w_{x}-\epsilon b_{5} x\left[\epsilon a_{11} w_{1, x}+\epsilon e Z_{x}+\epsilon^{2} \hat{\phi}_{x}+\phi_{4, x}\right] \\
& -\epsilon \beta\left(k_{1} f+f^{\prime}\right) \times\left\{\epsilon a_{12}(f+h) w_{2, x}+\epsilon e Z_{x}+\epsilon^{2} \hat{\phi}_{x}\right. \\
& \left.+\epsilon \beta^{-1}\left(A(0) Z_{x}+\phi_{22, x}(x, 0)\right)+\phi_{4, x}\right\} \\
& +\epsilon \alpha^{\prime} \alpha^{-1}\left\{\epsilon a_{11} w_{1}+\epsilon e Z+\epsilon^{2} \hat{\phi}+\phi_{4}\right\}+\epsilon^{2} k \alpha^{\prime} \alpha^{-1} \beta^{-1} x w \\
& +\epsilon^{2} \frac{k \alpha^{\prime}}{\alpha}\left(\frac{x}{\beta}+f+h\right) \times\left[\epsilon a_{11} w_{1}+\epsilon a_{12}(f+h) w_{2}\right. \\
& \left.\quad+\epsilon \beta^{-1}\left(A(0) Z+\phi_{22}(x, 0)\right)+\epsilon e Z+\epsilon^{2} \hat{\phi}+\phi_{4}\right] \\
& -\epsilon^{2}\left\{b_{1}\left(\beta^{-2} x^{2}+f^{2}+h^{2}+2 f h\right) \beta-k\left[\beta^{-2} \beta^{\prime} x^{2}-(f+h)\left(f^{\prime}+h^{\prime}\right) \beta\right]\right\} w_{x} \\
& -\epsilon^{3}\left[b_{1}\left(\frac{x}{\beta}+f+h\right)^{2} \beta-k\left(\frac{x}{\beta}+f+h\right)\left(\frac{\beta^{\prime}}{\beta} x-\beta f^{\prime}-\beta h^{\prime}\right)\right] \\
& \times\left[a_{11} w_{1, x}+a_{12}(f+h) w_{2, x}+\epsilon \beta^{-1}\left(A(0) Z_{x}+\phi_{22, x}\right)+e Z_{x}+\epsilon \hat{\phi}_{x}+\epsilon^{-1} \phi_{4, x}\right] \\
& +\epsilon^{2} a_{11}^{\prime} w_{1}+\epsilon^{2} e^{\prime} Z+\epsilon^{2} \hat{\phi}_{z}+\phi_{4, z}+\epsilon^{2} k \beta^{-2} x\left(\epsilon A^{\prime}(0) \beta(0) Z+\phi_{22, z}(x, 0)\right) \\
& +\epsilon k\left(\frac{x}{\beta}+f+h\right) \times\left[\epsilon^{2} a_{11}^{\prime} w_{1}+\epsilon^{2} a_{12}^{\prime}(f+h) w_{2}\right. \\
& \left.\quad+\epsilon^{2} a_{12}\left(f^{\prime}+h^{\prime}\right) w_{2}+\epsilon^{2} e^{\prime} Z+\epsilon^{2} \hat{\phi}_{z}+\phi_{4, z}\right] \\
& +D_{0}^{0}\left(v_{5}\right) . \\
& +\epsilon^{2} b_{2}\left(\frac{x}{\beta}+f+h\right)^{2} \times\left[\epsilon^{2} a_{12}^{\prime} w_{1}+\epsilon^{2} a_{12}^{\prime}(f+h) w_{2}+\epsilon^{2} a_{12}\left(f^{\prime}+h^{\prime}\right) w_{2}\right.
\end{aligned}
$$

The term $g_{1}$ has a similar expression.

We decompose

$$
\mathcal{E}=\mathcal{E}_{11}+\mathcal{E}_{12}, \quad g_{0}=g_{01}+g_{02}, \quad g_{1}=g_{11}+g_{12},
$$

with

$$
\begin{aligned}
\mathcal{E}_{11}=\epsilon^{3} \beta^{-2} e^{\prime \prime} Z+\epsilon \lambda_{0} e Z, & \text { and } & \mathcal{E}_{12} & =\mathcal{E}-\mathcal{E}_{11}, \\
g_{01}=-\epsilon\left[k_{1} \beta(0) f+\beta(0) f^{\prime}\right] w_{x}+\epsilon^{2} e^{\prime} Z, & \text { and } & g_{02} & =g_{0}-g_{01}, \\
g_{11}=-\epsilon\left[k_{2} \beta(1) f+\beta(1) f^{\prime}\right] w_{x}+\epsilon^{2} e^{\prime} Z, & \text { and } & g_{12} & =g_{1}-g_{11} .
\end{aligned}
$$

For further references, it is useful to estimate the $L^{2}(\mathcal{S})$ norm of $\mathcal{E}$. From the uniform bound of $e$ in (3.50), it is easy to see that

$$
\left\|\mathcal{E}_{11}\right\|_{L^{2}(\mathcal{S})} \leq C \epsilon^{1 / 2}
$$


Since $\phi_{1}, \phi_{2}$ and $\epsilon e Z$ are of size $O(\epsilon)$, all terms in $\mathcal{E}_{12}$ carry $\epsilon^{2}$ in front. We claim that

$$
\left\|\mathcal{E}_{12}\right\|_{L^{2}(\mathcal{S})} \leq C \epsilon^{3 / 2} .
$$

A rather delicate term in $\mathcal{E}_{12}$ is the one carrying $f^{\prime \prime}$ since we only assume a uniform bound on $\left\|f^{\prime \prime}\right\|_{L^{2}(0,1)}$. For example, we have a term $K_{1}=\epsilon^{2} f^{\prime \prime}$ in $S(w)$ which has bound like

$$
\left\|K_{1}\right\|_{L^{2}(\mathcal{S})} \leq C \epsilon^{2}
$$

Since

$$
\begin{aligned}
& \left|N_{0}\left(\phi_{1}+\phi_{2}+\epsilon e z+\phi_{3}+\phi_{4}\right)\right| \\
& =\left|\left(w+\phi_{1}+\phi_{2}+\epsilon e z+\phi_{3}+\phi_{4}\right)^{p}-w^{p}-p w^{p-1}\left(\phi_{1}+\phi_{2}+\epsilon e z+\phi_{3}+\phi_{4}\right)\right| \\
& =\left|p\left[w+t\left(\phi_{1}+\phi_{2}+\epsilon e z+\phi_{3}+\phi_{4}\right)\right]^{p-2}\left(\phi_{1}+\phi_{2}+\epsilon e z+\phi_{3}+\phi_{4}\right)^{2}\right|
\end{aligned}
$$

we obtain

$$
\left\|N_{0}\left(\phi_{1}+\phi_{2}+\epsilon e z+\phi_{3}+\phi_{4}\right)\right\|_{L^{2}(\mathcal{S})} \leq C \epsilon^{3 / 2} .
$$

Other terms can be estimated in the similar way. Similarly, we have the following estimate

$$
\left\|g_{02}\right\|_{L^{2}(\mathbb{R})}+\left\|g_{12}\right\|_{L^{2}(\mathbb{R})} \leq C \epsilon^{3 / 2}
$$

Moreover, for the Lipschitz dependence of the term of error $\mathcal{E}_{12}$ on the parameters $f$ and $e$ for the norm defined in (3.23) and (3.50), we have the validity of the estimate

$$
\left\|\mathcal{E}_{12}\left(f_{1}, e_{1}\right)-\mathcal{E}_{12}\left(f_{2}, e_{2}\right)\right\|_{L^{2}(\mathcal{S})} \leq C \epsilon^{3 / 2}\left[\left\|f_{1}-f_{2}\right\|_{*}+\left\|e_{1}-e_{2}\right\|_{* *}\right] .
$$

Similarly, we obtain

$$
\begin{aligned}
\left\|g_{02}\left(f_{1}, e_{1}\right)-g_{02}\left(f_{2}, e_{2}\right)\right\|_{L^{2}(\mathbb{R})}+ & \left\|g_{12}\left(f_{1}, e_{1}\right)-g_{12}\left(f_{2}, e_{2}\right)\right\|_{L^{2}(\mathbb{R})} \\
& \leq C \epsilon^{3 / 2}\left[\left\|f_{1}-f_{2}\right\|_{*}+\left\|e_{1}-e_{2}\right\|_{* *}\right] .
\end{aligned}
$$

\section{The resolution theory for the projected problem}

\section{$5.1 \quad$ The invertibility of $\mathcal{L}$}

Let $\mathcal{L}, \mathcal{D}_{1}$ and $\mathcal{D}_{0}$ be the operators defined in $H^{2}(\mathcal{S})$ by $(3.51)-(3.53)$, and also $g_{0}, g_{1}$ be the functions in (4.73). Note that the function $\chi(\epsilon|x|)$ is even in the definition of $\mathcal{L}$. In this section, we study the following linear problem: for given $\mathfrak{h} \in L^{2}(\mathcal{S}), g_{0}, g_{1} \in L^{2}(\mathbb{R})$, finding functions $\phi \in H^{2}(\mathcal{S}), c, d \in L^{2}(0,1)$ and constants $l_{1}, l_{0}, m_{1}, m_{0}$ such that

$$
\begin{array}{cl}
\mathcal{L}(\phi)=\mathfrak{h}+c(\epsilon z) \chi(\epsilon|x|) w_{x}+d(\epsilon z) \chi(\epsilon|x|) Z & \text { in } \mathcal{S}, \\
\mathcal{D}_{1}(\phi)=\eta_{\delta}^{\epsilon}(s) g_{1}+l_{1} \chi(\epsilon|x|) w_{x}+m_{1} \chi(\epsilon|x|) Z & \text { on } \partial_{1} \mathcal{S}, \\
\mathcal{D}_{0}(\phi)=\eta_{\delta}^{\epsilon}(s) g_{0}+l_{0} \chi(\epsilon|x|) w_{x}+m_{0} \chi(\epsilon|x|) Z & \text { on } \partial_{0} \mathcal{S}, \\
\int_{\mathbb{R}} \phi(x, z) w_{x}(x) \mathrm{d} x=0, \quad \int_{\mathbb{R}} \phi(x, z) Z(x) \mathrm{d} x=0, & 0<z<\frac{1}{\epsilon} .
\end{array}
$$


Proposition 5.1. If $\delta$ in the definition of $\mathcal{L}$ is chosen small enough and $\mathfrak{h} \in L^{2}(\mathcal{S})$, there exists a constant $C>0$, independent of $\epsilon$, such that for all small $\epsilon$, the problem (5.1)-(5.4) has a unique solution $\phi=\mathbf{T}(\mathfrak{h})$ which satisfies

$$
\|\phi\|_{H^{2}(\mathcal{S})} \leq C\left(\|\mathfrak{h}\|_{L^{2}(\mathcal{S})}+\left\|g_{02}\right\|_{L^{2}(\mathbb{R})}+\left\|g_{12}\right\|_{L^{2}(\mathbb{R})}\right),
$$

Moreover, if $h, g_{0}, g_{1}$ have compact supports contained in $|x| \leq 20 \delta / \epsilon$, then

$$
|\phi(x, z)|+|\nabla \phi(x, z)| \leq\|\phi\|_{L^{\infty}} e^{-2 \delta / \epsilon} \quad \text { for }|x|>40 \delta / \epsilon .
$$

Proof. Note that $\eta_{\delta}^{\epsilon}(s) g_{11}$ and $\eta_{\delta}^{\epsilon}(s) g_{01}$ can be absorbed by $l_{1} \chi w_{x}+m_{1} \chi Z$ and $l_{0} \chi w_{x}+m_{0} \chi Z$, the problem (5.1)-(5.4) can be written as

$$
\begin{array}{cl}
\mathcal{L}(\phi)=\mathfrak{h}+c(\epsilon z) \chi(\epsilon|x|) w_{x}+d(\epsilon z) \chi(\epsilon|x|) Z & \text { in } \mathcal{S}, \\
\mathcal{D}_{1}(\phi)=\eta_{\delta}^{\epsilon}(s) g_{12}+l_{1} \chi(\epsilon|x|) w_{x}+m_{1} \chi(\epsilon|x|) Z & \text { on } \partial_{1} \mathcal{S}, \\
\mathcal{D}_{0}(\phi)=\eta_{\delta}^{\epsilon}(s) g_{02}+l_{0} \chi(\epsilon|x|) w_{x}+m_{0} \chi(\epsilon|x|) Z & \text { on } \partial_{0} \mathcal{S}, \\
\int_{\mathbb{R}} \phi(x, z) w_{x}(x) \mathrm{d} x=0, \quad \int_{\mathbb{R}} \phi(x, z) Z(x) \mathrm{d} x=0, & 0<z<\frac{1}{\epsilon} .
\end{array}
$$

We will reduce problem (5.7)-(5.10) to a small perturbed problem in which Lemma 2.5 in [61] is applicable. We will achieve this by introducing a change of variables that eliminates the weight $\beta^{-2}$ in front of $\phi_{z z}$.

We let

$$
\phi(x, z)=\varphi(x, \tilde{z}) \quad \text { with } \quad \tilde{z}=\Upsilon(z),
$$

where the map $\Upsilon:\left[0, \frac{1}{\epsilon}\right) \rightarrow\left[0, \frac{\ell}{\epsilon}\right)$ is a diffeomorphism defined in (4.28) with the constant $\ell$ given in (4.23). We then have

$$
\phi_{z}=\beta(\epsilon z) \varphi_{\tilde{z}}, \quad \phi_{z z}=\beta^{2}(\epsilon z) \varphi_{\tilde{z} \tilde{z}}+\epsilon \beta^{\prime}(\epsilon z) \varphi_{\tilde{z}},
$$

while the differentiation in $x$ does not change. Similarly, for any function $g$, we set

$$
\tilde{g}(x, \tilde{z})=g\left(x, \Upsilon^{-1}(\tilde{z})\right) .
$$

The equation in terms of $\varphi$ now reads

$$
\begin{aligned}
\Delta \varphi-\varphi+p w^{p-1} \varphi= & -p\left(\tilde{\beta}^{-2} \chi(\epsilon|x|) \tilde{\mathbf{W}}^{p-1}-w^{p-1}\right) \varphi-\chi(\epsilon|x|) \tilde{B}_{3}(\varphi)-\epsilon \widetilde{\beta}^{\prime} \tilde{\beta}^{-2} \varphi_{\tilde{z}} \\
& +\tilde{\mathfrak{h}}+\tilde{c}(\epsilon \tilde{z}) \chi(\epsilon|x|) w_{x}+\tilde{d}(\epsilon \tilde{z}) \chi(\epsilon|x|) Z \quad \text { in } \hat{\mathcal{S}},
\end{aligned}
$$

with the boundary conditions

$$
\begin{aligned}
& \varphi_{\tilde{z}}=\beta^{-1}\left[\mathbb{G}_{1}(\varphi)-l_{1} \chi(\epsilon|x|) w_{x}-m_{1} \chi(\epsilon|x|) Z\right] \quad \text { on } \partial_{1} \hat{\mathcal{S}}, \\
& \varphi_{\tilde{z}}=\beta^{-1}\left[\mathbb{G}_{0}(\varphi)-l_{0} \chi(\epsilon|x|) w_{x}-m_{0} \chi(\epsilon|x|) Z\right] \quad \text { on } \partial_{0} \hat{\mathcal{S}},
\end{aligned}
$$

where

$$
\begin{aligned}
& \mathbb{G}_{1}(\varphi)=-\widetilde{\eta}_{\delta}^{\epsilon} \tilde{g}_{12}+\chi(\epsilon|x|) \tilde{D}_{3}^{1}(\varphi)+\chi(\epsilon|x|) \tilde{D}_{0}^{1}(\varphi), \\
& \mathbb{G}_{0}(\varphi)=-\widetilde{\eta}_{\delta}^{\epsilon} \tilde{g}_{02}+\chi(\epsilon|x|) \tilde{D}_{3}^{0}(\varphi)+\chi(\epsilon|x|) \tilde{D}_{0}^{0}(\varphi),
\end{aligned}
$$


and the orthogonality condition

$$
\int_{\mathbb{R}} \varphi(x, \tilde{z}) w_{x}(x) \mathrm{d} x=\int_{\mathbb{R}} \varphi(x, \tilde{z}) Z(x) \mathrm{d} x=0, \quad 0<\tilde{z}<\frac{\ell}{\epsilon} .
$$

The operators $\tilde{B}_{3}, \tilde{D}_{3}^{i}, \tilde{D}_{0}^{i}$ are defined by using the above formulas to replace the $z$-derivatives by $\tilde{z}$-derivatives and the variable $z$ by $\Upsilon^{-1}(\tilde{z})$ in the operators $B_{3}, D_{3}^{i}, D_{0}^{i}$.

The key point is the following: the operator

$$
B_{4}(\varphi)=p\left(\tilde{\beta}^{-2} \chi(\epsilon|x|) \tilde{\mathbf{W}}^{p-1}-w^{p-1}\right) \varphi+\chi(\epsilon|x|) \tilde{B}_{3}(\varphi)+\epsilon \widetilde{\beta}^{\prime} \tilde{\beta}^{-2} \varphi_{\tilde{z}},
$$

is small in the sense that

$$
\left\|B_{4}(\varphi)\right\|_{L^{2}(\hat{\mathcal{S}})} \leq C \delta\|\varphi\|_{H^{2}(\hat{\mathcal{S}})} .
$$

This last estimate is a rather straightforward consequence of the fact that $|s| \leq 20 \delta \epsilon^{-1}$ wherever the operator $\tilde{B}_{3}$ is supported, and the other terms are even smaller when $\epsilon$ is small. Similar results hold for $\mathbb{G}_{1}(\varphi)$ and $\mathbb{G}_{0}(\varphi)$. Thus by reducing $\delta$ if necessary, we apply the invertibility result of Lemma 2.5 in [61]. The result thus follows by transforming the estimate for $\varphi$ into similar one for $\phi$ via change of variables. This concludes the proof.

\subsection{Solving the nonlinear projection problem}

In this section, we will solve (3.56)-(3.59) in $\mathcal{S}$. A first elementary, but crucial observation is that: The term

$$
\mathcal{E}_{11}=\left(\epsilon^{3} \beta^{-2} e^{\prime \prime}+\epsilon \lambda_{0} e\right) Z,
$$

in the decomposition of $\mathcal{E}$, has precisely the form $d(\epsilon z) Z$ and can be absorbed in that term $\chi(\epsilon|x|) d(\epsilon z) Z$. Then, the equivalent equation of (3.56) is

$$
\mathcal{L}(\phi)=-\eta_{\delta}^{\epsilon}(s) \mathcal{E}_{12}-\eta_{\delta}^{\epsilon}(s) \mathcal{N}(\phi)+c(\epsilon z) \chi(\epsilon|x|) w_{x}+d(\epsilon z) \chi(\epsilon|x|) Z .
$$

Similarly, we can also absorb the terms of order $O(\epsilon)$ in $g_{0}$ and $g_{1}$.

Let $\mathbf{T}$ be the bounded operator defined by Proposition 5.1. Then the problem (3.56)-(3.59) is equivalent to the following fixed point problem

$$
\phi=\mathbf{T}\left(-\eta_{\delta}^{\epsilon}(s) \mathcal{E}_{12}-\eta_{\delta}^{\epsilon}(s) \mathcal{N}(\phi)\right) \equiv \mathcal{A}(\phi) .
$$

We collect some useful facts to find the domain of the operator $\mathcal{A}$ such that $\mathcal{A}$ becomes a contraction mapping.

The big difference between $\mathcal{E}_{11}$ and $\mathcal{E}_{12}$ is their size. From (4.76) and (4.77)

$$
\left\|\mathcal{E}_{12}\right\|_{L^{2}(\mathcal{S})} \leq c_{*} \epsilon^{3 / 2},
$$

while $\mathcal{E}_{11}$ is only of size $O\left(\epsilon^{1 / 2}\right)$. Similarly, we have

$$
\left\|g_{02}\right\|_{L^{2}(\mathbb{R})}+\left\|g_{12}\right\|_{L^{2}(\mathbb{R})} \leq c_{*} \epsilon^{3 / 2} .
$$

On the other hand, the operator $\mathbf{T}$ has a useful property: assuming $\bar{h}$ has a support contained in $|x| \leq 20 \delta / \epsilon$, then $\phi=\mathbf{T}(\bar{h})$ satisfies the estimate

$$
|\phi(x, z)|+|\nabla \phi(x, z)| \leq\|\phi\|_{L^{\infty}} e^{-2 \delta / \epsilon} \text { for }|x|>40 \delta / \epsilon .
$$


Recall that the operator $\psi(\phi)$ satisfies, as seen directly from its definition

$$
\|\psi(\phi)\|_{L^{\infty}} \leq C \epsilon\left[\||\phi|+|\nabla \phi|\|_{L^{\infty}(|x|>20 \delta / \epsilon)}+e^{-\delta / \epsilon}\right],
$$

and a Lipschitz condition of the form

$$
\left\|\psi\left(\phi_{1}\right)-\psi\left(\phi_{2}\right)\right\|_{L^{\infty}} \leq C \epsilon\left[\left\|\left|\phi_{1}-\phi_{2}\right|+\left|\nabla\left(\phi_{1}-\phi_{2}\right)\right|\right\|_{L^{\infty}(|x|>20 \delta / \epsilon)}\right] .
$$

Now, the facts above will allow us to construct a region where contraction mapping principle applies and then solve the problem (3.56)-(3.59).

Consider the following closed, bounded subset

$$
\Xi=\left\{\phi \in H^{2}(\mathcal{S})\left|\|\phi\|_{H^{2}(\mathcal{S})} \leq \tau \epsilon^{3 / 2}, \quad\||\phi|+|\nabla \phi|\|_{L^{\infty}(|x|>40 \delta / \epsilon)} \leq\|\phi\|_{H^{2}(\mathcal{S})} e^{-\delta / \epsilon}\right\} .\right.
$$

If the constant $\tau$ is sufficiently large, then the map $\mathcal{A}$ defined in (5.14) is a contraction from $\Xi$ into itself. This property will lead to the following proposition:

Proposition 5.2. There is a number $\tau>0$ such that for all $\epsilon$ small enough satisfying gap condition (1.14) and all parameters $(f, e)$ in $\mathcal{F}$, problem (3.56)-(3.59) has a unique solution $\phi=\phi(f, e)$ which satisfies

$$
\begin{gathered}
\|\phi\|_{H^{2}(\mathcal{S})} \leq \tau \epsilon^{3 / 2} \\
\||\phi|+|\nabla \phi|\|_{L^{\infty}(|x|>40 \delta / \epsilon)} \leq\|\phi\|_{H^{2}(\mathcal{S})} e^{-\delta / \epsilon} .
\end{gathered}
$$

Moreover, $\phi$ depends Lipschitz-continuously on the parameters $f$ and $e$ in the sense of the estimate

$$
\left\|\phi\left(f_{1}, e_{1}\right)-\phi\left(f_{2}, e_{2}\right)\right\|_{H^{2}(\mathcal{S})} \leq C \epsilon^{3 / 2}\left[\left\|f_{1}-f_{2}\right\|_{*}+\left\|e_{1}-e_{2}\right\|_{* *}\right] .
$$

Proof. The proof is similar as that for Proposition 5.1 in [23].

\section{Estimates of the projection against $w_{x}$ and $Z$}

As we have mentioned in Section 3, in the next part of the paper, we will set up equations for the parameters $f$ and $e$ which are equivalent to making $c(\epsilon z), d(\epsilon z), l_{1}, l_{0}, m_{1}$ and $m_{0}$ are identically zero in the system (3.56)-(3.59). To achieve this, we first multiply the equation against $w_{x}$ only in $x$. The equations

$$
c(\epsilon z)=0, \quad l_{1}=0, \quad l_{0}=0,
$$

are then equivalent to the relations

$$
\begin{gathered}
\int_{\mathbb{R}}\left[\eta_{\delta}^{\epsilon}(s) \mathcal{E}+\eta_{\delta}^{\epsilon}(s) \mathcal{N}(\phi)+\chi(\epsilon|x|) B_{3}(\phi)+p\left(\beta^{-2} \chi(\epsilon|x|) \mathbf{W}^{p-1}-w^{p-1}\right) \phi\right] w_{x} \mathrm{~d} x=0, \\
\int_{\mathbb{R}}\left[\eta_{\delta}^{\epsilon}(s) g_{1}+\chi(\epsilon|x|) D_{3}^{1}(\phi)+\chi(\epsilon|x|) D_{0}^{1}(\phi)\right] w_{x} \mathrm{~d} x=0, \quad z=1 / \epsilon, \\
\int_{\mathbb{R}}\left[\eta_{\delta}^{\epsilon}(s) g_{0}+\chi(\epsilon|x|) D_{3}^{0}(\phi)+\chi(\epsilon|x|) D_{0}^{1}(\phi)\right] w_{x} \mathrm{~d} x=0, \quad z=0 .
\end{gathered}
$$


Similarly,

$$
d(\epsilon z)=0, \quad m_{1}=0, \quad m_{0}=0,
$$

if and only if

$$
\begin{gathered}
\int_{\mathbb{R}}\left[\eta_{\delta}^{\epsilon}(s) \mathcal{E}+\eta_{\delta}^{\epsilon}(s) \mathcal{N}(\phi)+\chi(\epsilon|x|) B_{3}(\phi)+p\left(\beta^{-2} \chi(\epsilon|x|) \mathbf{W}^{p-1}-w^{p-1}\right) \phi\right] Z \mathrm{~d} x=0, \\
\int_{\mathbb{R}}\left[\eta_{\delta}^{\epsilon}(s) g_{1}+\chi(\epsilon|x|) D_{3}^{1}(\phi)+\chi(\epsilon|x|) D_{0}^{1}(\phi)\right] Z \mathrm{~d} x=0, \quad z=1 / \epsilon, \\
\int_{\mathbb{R}}\left[\eta_{\delta}^{\epsilon}(s) g_{0}+\chi(\epsilon|x|) D_{3}^{0}(\phi)+\chi(\epsilon|x|) D_{0}^{1}(\phi)\right] Z \mathrm{~d} x=0, \quad z=0 .
\end{gathered}
$$

It is therefore of crucial importance to carry out computation of the estimates of the terms

$$
\int_{\mathbb{R}} \mathcal{E} w_{x} \mathrm{~d} x \text { and } \int_{\mathbb{R}} \mathcal{E} Z \mathrm{~d} x
$$

and, similarly, some other terms involving $\phi$.

\subsection{Estimates for projections of the error}

In this section, we carry out some estimates for the terms

$$
\int_{\mathbb{R}} \mathcal{E} w_{x} \mathrm{~d} x \text { and } \int_{\mathbb{R}} \mathcal{E} Z \mathrm{~d} x .
$$

For the pair $(f, e)$ satisfying (3.23) and (3.50), we denote $\mathbf{b}_{1 \epsilon}$ and $\mathbf{b}_{2 \epsilon}$, generic, uniformly bounded continuous functions of the form

$$
\mathbf{b}_{l \epsilon}=\mathbf{b}_{l \epsilon}\left(z, f(\epsilon z), e(\epsilon z), f^{\prime}(\epsilon z), \epsilon e^{\prime}(\epsilon z)\right), l=1,2
$$

where $\mathbf{b}_{1 \epsilon}$ is uniformly Lipschitz in its four last arguments.

\subsection{1}

First, we estimates for the term $\int_{\mathbb{R}} \mathcal{E} w_{x} \mathrm{~d} x$, where $\mathcal{E}$, we recall, was defined in (4.72) and $w_{x}$ is an odd function. Integration against all even terms, say $\mathcal{E}_{11}$ and $S_{4}, M_{12}, M_{22}$ in $\mathcal{E}_{12}$, therefore just vanish. We have

$$
\begin{aligned}
\int_{\mathbb{R}} \mathcal{E} w_{x} \mathrm{~d} x= & \int_{\mathbb{R}} \mathcal{E}_{12} w_{x} \mathrm{~d} x \\
= & \int_{\mathbb{R}} \epsilon^{2} S_{3} w_{x} \mathrm{~d} x+\int_{\mathbb{R}} \epsilon^{2} S_{5} w_{x} \mathrm{~d} x+\int_{\mathbb{R}} B_{2}(w) w_{x} \mathrm{~d} x+\int_{\mathbb{R}} \beta^{-2} \phi_{1, z z} w_{x} \mathrm{~d} x \\
& +\int_{\mathbb{R}} \beta^{-2} \phi_{4, z z} w_{x} \mathrm{~d} x+\int_{\mathbb{R}} M_{32}(x, z) w_{x} \mathrm{~d} x+\int_{\mathbb{R}} M_{42}(x, z) w_{x} \mathrm{~d} x \\
& +\int_{\mathbb{R}} M_{52}(x, z) w_{x} \mathrm{~d} x+\int_{\mathbb{R}} M_{62}(x, z) w_{x} \mathrm{~d} x+\int_{\mathbb{R}}\left[B_{3}\left(\phi_{3}\right)+B_{3}\left(\phi_{4}\right)\right] w_{x} \mathrm{~d} x \\
\equiv & \mathrm{I}_{1}+\mathrm{I}_{2}+\mathrm{I}_{3}+\mathrm{I}_{4}+\mathrm{I}_{5}+\mathrm{I}_{6}+\mathrm{I}_{7}+\mathrm{I}_{8}+\mathrm{I}_{9}+\mathrm{I}_{10} .
\end{aligned}
$$

These terms will be estimated as follows. 
By repeating the same computation used in (4.54) and (4.56), we get

$$
\begin{aligned}
\mathrm{I}_{1} & =\int_{\mathbb{R}} \epsilon^{2} S_{3} w_{x} \mathrm{~d} x \\
& =-\epsilon^{2} \varrho_{1} \beta^{-1} f^{\prime \prime}-\epsilon^{2} \varrho_{1} \beta^{-1}\left(\beta^{-1} \beta^{\prime}+2 \alpha^{-1} \alpha^{\prime}\right) f^{\prime}-\epsilon^{2} \varrho_{1} \beta^{-1}\left(k^{2}-\sigma \beta^{-2} V_{t t}\right) f
\end{aligned}
$$

and also

$$
\mathrm{I}_{2}=\int_{\mathbb{R}} \epsilon^{2} S_{5} w_{x} \mathrm{~d} x=-\epsilon^{2} \varrho_{1} \beta^{-1}\left[\varpi \beta^{\prime} \beta^{-1}+2 \varpi \alpha^{-1} \alpha^{\prime}\right] f .
$$

Recall the expression of $B_{2}(w)$ in (3.30) and $\phi_{1}$, it is easy to check that

$$
\mathrm{I}_{3}+\mathrm{I}_{4}=\int_{\mathbb{R}} B_{2}(w) w_{x} \mathrm{~d} x+\int_{\mathbb{R}} \beta^{-2} \phi_{1, z z} w_{x} \mathrm{~d} x=\epsilon^{3} \mathbf{b}_{1 \epsilon} f^{\prime \prime}+\epsilon^{3} \mathbf{b}_{2 \epsilon} .
$$

Since

$$
\phi_{4}(x, z)=\epsilon^{2} \phi_{41}(x, \epsilon z)+\epsilon^{2} \phi_{42}(x, \epsilon z)
$$

it can be derived that

$$
\mathrm{I}_{5}=\int_{\mathbb{R}} \beta^{-2} \phi_{4, z z} w_{x} \mathrm{~d} x=O\left(\epsilon^{4}\right) .
$$

Since $w_{x}$ is odd in $x$, we need only consider the odd terms in $M_{32}(x, z)$ and get

$$
\begin{aligned}
\mathrm{I}_{6} & =\int_{\mathbb{R}} M_{32}(x, z) w_{x} \mathrm{~d} x \\
& =\epsilon^{2} \beta^{-1} \sigma^{-1} k^{2} f \int_{\mathbb{R}}\left[w_{2, x} w_{x}+\frac{1}{\sigma} x w_{x} w_{2}+w_{1} w_{x}\right] \mathrm{d} x+O\left(\epsilon^{3}\right) .
\end{aligned}
$$

From the definition of $M_{42}(x, z)$, we can estimate the $I_{7}$ in (6.7) as the following

$$
\begin{aligned}
\mathrm{I}_{7}= & \int_{\mathbb{R}} M_{42}(x, z) w_{x} \mathrm{~d} x \\
= & -\frac{2 \epsilon^{2}}{\beta} f^{\prime} \xi(\epsilon z) \int_{\mathbb{R}}\left[\epsilon A^{\prime}(\mathfrak{a}(\epsilon z)) \beta Z_{x}+\phi_{22, x z}\right] w_{x} \mathrm{~d} x \\
& -\frac{2 \epsilon^{2}}{\beta} \varpi f \xi(\epsilon z) \int_{\mathbb{R}}\left[\epsilon A^{\prime}(\mathfrak{a}(\epsilon z)) \beta Z_{x}+\phi_{22, x z}\right] w_{x} \mathrm{~d} x+\epsilon^{3} \mathbf{b}_{1 \epsilon} f^{\prime \prime}+\epsilon^{3} \mathbf{b}_{2 \epsilon} \\
\equiv & \epsilon^{2} \varrho_{1} \beta^{-1} \alpha_{1}(z) f^{\prime}+\epsilon^{2} \varrho_{1} \beta^{-1} \alpha_{2}(z) f+\epsilon^{3} \mathbf{b}_{1 \epsilon} f^{\prime \prime}+\epsilon^{3} \mathbf{b}_{2 \epsilon},
\end{aligned}
$$

where $\alpha_{1}(z)$ and $\alpha_{2}(z)$ are defined in (4.59)-(4.60).

From the definition of $M_{52}(x, z)$, we need only consider the odd terms and the higher order terms involving $e^{\prime}$ and $e^{\prime \prime}$, so we get

$$
\begin{aligned}
\mathrm{I}_{8}=-\epsilon^{2} k \beta^{-1} e \int_{\mathbb{R}}\left[Z_{x}+\sigma^{-1} x Z\right] w_{x} \mathrm{~d} x+2 \epsilon^{4} k \beta^{-3} e^{\prime \prime}(\epsilon z) \int_{\mathbb{R}} x w_{x} Z(x) \mathrm{d} x \\
\quad+\epsilon^{3}\left[\mathbf{b}_{1 \epsilon}^{1} e^{\prime}+\mathbf{b}_{1 \epsilon} f^{\prime \prime}+\mathbf{b}_{2 \epsilon}\right] \\
\equiv \epsilon^{2} \varrho_{1} \beta^{-1}\left[\hbar_{31}(\epsilon z) e+\epsilon^{2} \hbar_{4}(\epsilon z) e^{\prime \prime}\right]+\epsilon^{3}\left[\mathbf{b}_{1 \epsilon}^{1} e^{\prime}+\mathbf{b}_{1 \epsilon} f^{\prime \prime}+\mathbf{b}_{2 \epsilon}\right] .
\end{aligned}
$$


It can easily be verified that

$$
\begin{aligned}
\mathrm{I}_{9}= & \int_{\mathbb{R}} M_{62}(x, z) w_{x} \mathrm{~d} x \\
= & \epsilon^{2} \beta^{-1} \sigma^{-1} k^{2} f \int_{\mathbb{R}} p(p-1) w_{x} w^{p-2} w_{1} w_{2} \mathrm{~d} x \\
& +\epsilon^{2} p(p-1) a_{11} e \int_{\mathbb{R}} w^{p-2} w_{1} Z w_{x} \mathrm{~d} x+\epsilon^{3} \mathbf{b}_{2 \epsilon} . \\
= & \epsilon^{2} \beta^{-1} \sigma^{-1} k^{2} f \int_{\mathbb{R}} p(p-1) w_{x} w^{p-2} w_{1} w_{2} \mathrm{~d} x+\epsilon^{2} \varrho_{1} \beta^{-1} \hbar_{32}(\epsilon z) e+\epsilon^{3} \mathbf{b}_{2 \epsilon}
\end{aligned}
$$

According to the fact that the terms in $B_{3}\left(\phi_{3}\right)$ and $B_{3}\left(\phi_{4}\right)$ are of order $O\left(\epsilon^{3}\right)$, it follows that

$$
\mathrm{I}_{10}=\int_{\mathbb{R}}\left[B_{3}\left(\phi_{3}\right)+B_{3}\left(\phi_{4}\right)\right] w_{x} \mathrm{~d} x=\epsilon^{3} \mathbf{b}_{1 \epsilon} f^{\prime \prime}+\epsilon^{3} \mathbf{b}_{2 \epsilon} .
$$

Adding (6.12) and (6.15), we get

$$
\begin{aligned}
& \mathrm{I}_{6}+\mathrm{I}_{9}=\int_{\mathbb{R}} M_{32}(x, z) w_{x} \mathrm{~d} x+\int_{\mathbb{R}} M_{62}(x, z) w_{x} \mathrm{~d} x \\
&=\epsilon^{2} \beta^{-1} \sigma^{-1} k^{2} f \int_{\mathbb{R}}[ p(p-1) w^{p-2} w_{x} w_{1} w_{2}+w_{2, x} w_{x}+\sigma^{-1} x w_{x} w_{2} \\
&\left.\quad+w_{1} w_{x}\right] \mathrm{d} x+\epsilon^{2} \varrho_{1} \beta^{-1} \hbar_{32}(\epsilon z) e+\left[\epsilon^{3} \mathbf{b}_{1 \epsilon} f^{\prime \prime}+\epsilon^{3} \mathbf{b}_{2 \epsilon}\right] \\
&=-\epsilon^{2} \varrho_{1} \beta^{-1} \sigma^{-1} k^{2} f+\epsilon^{2} \varrho_{1} \beta^{-1} \hbar_{32}(\epsilon z) e+\epsilon^{3}\left[\mathbf{b}_{1 \epsilon} f^{\prime \prime}+\mathbf{b}_{2 \epsilon}\right] .
\end{aligned}
$$

This leads to the conclusion of this section: there holds

$$
\begin{aligned}
\int_{\mathbb{R}} \mathcal{E} w_{x} \mathrm{~d} x= & -\epsilon^{2} \varrho_{1} \beta^{-1}\left\{f^{\prime \prime}+\left[\hbar_{1}(\epsilon z)+\alpha_{1}(z)\right] f^{\prime}+\left[\hbar_{2}(\epsilon z)+\alpha_{2}(z)\right] f\right\} \\
& +\epsilon^{2} \varrho_{1} \beta^{-1}\left[\hbar_{3}(\epsilon z) e+\epsilon^{2} \hbar_{4}(\epsilon z) e^{\prime \prime}\right]+\epsilon^{3}\left[\mathbf{b}_{1 \epsilon}^{1} e^{\prime}+\mathbf{b}_{1 \epsilon}^{2} f^{\prime \prime}+\mathbf{b}_{2 \epsilon}\right],
\end{aligned}
$$

the functions $\hbar_{1}$ and $\hbar_{2}$ are defined in (4.67)-(4.68), $\hbar_{3}=\hbar_{31}+\hbar_{32}$.

\section{1 .2}

In the following, we are in a position to provide the concise estimate for the integral $\int_{\mathbb{R}} \mathcal{E} Z \mathrm{~d} x$. Using the decomposition of $\mathcal{E}$, we obtain

$$
\int_{\mathbb{R}} \mathcal{E} Z \mathrm{~d} x=\int_{\mathbb{R}} \mathcal{E}_{11} Z \mathrm{~d} x+\int_{\mathbb{R}} \mathcal{E}_{12} Z \mathrm{~d} x
$$

where

$$
\int_{\mathbb{R}} \mathcal{E}_{11} Z \mathrm{~d} x=\epsilon\left[\epsilon^{2} \beta^{-2} e^{\prime \prime}+\lambda_{0} e\right] \int_{\mathbb{R}} Z^{2} \mathrm{~d} x=\epsilon^{3} \beta^{-2} e^{\prime \prime}+\epsilon \lambda_{0} e
$$


and

$$
\begin{aligned}
\int_{\mathbb{R}} \mathcal{E}_{12} Z \mathrm{~d} x= & \int_{\mathbb{R}} \epsilon^{2} S_{4} Z \mathrm{~d} x+\int_{\mathbb{R}} B_{2}(w) Z \mathrm{~d} x+\int_{\mathbb{R}} \beta^{-2} \phi_{1, z z} Z \mathrm{~d} x \\
& +\int_{\mathbb{R}} \beta^{-2} \phi_{4, z z} Z \mathrm{~d} x+\int_{\mathbb{R}} M_{12}(x, z) Z \mathrm{~d} x+\int_{\mathbb{R}} M_{22}(x, z) Z \mathrm{~d} x \\
& +\int_{\mathbb{R}} M_{32}(x, z) Z \mathrm{~d} x+\int_{\mathbb{R}} M_{42}(x, z) Z \mathrm{~d} x+\int_{\mathbb{R}} M_{52}(x, z) Z \mathrm{~d} x \\
& +\int_{\mathbb{R}} M_{62}(x, z) Z \mathrm{~d} x+\int_{\mathbb{R}} B_{3}\left(\phi_{3}\right) Z \mathrm{~d} x+\int_{\mathbb{R}} B_{3}\left(\phi_{4}\right) Z \mathrm{~d} x \\
\equiv & \mathrm{II}_{1}+\mathrm{II}_{2}+\mathrm{II}_{3}+\mathrm{II}_{4}+\mathrm{II}_{5}+\mathrm{II}_{6}+\mathrm{II}_{7}+\mathrm{II}_{8}+\mathrm{II}_{9}+\mathrm{II}_{10}+\mathrm{II}_{11}+\mathrm{II}_{12} .
\end{aligned}
$$

According to the expression of $S_{4}$ in (4.2) and the constraint of $f$ in (3.23), it follows that

$$
\begin{aligned}
& \mathrm{II}_{1}=\int_{\mathbb{R}} \epsilon^{2} S_{4} Z \mathrm{~d} x \\
& =\epsilon^{2} \int_{\mathbb{R}}\left[\left(f^{\prime 2}+2 f^{\prime} h^{\prime}\right) w_{x x}+2 \varpi\left(f f^{\prime}+f^{\prime} h\right) w_{x x}-\frac{1}{2 \beta^{2}} V_{t t} f^{2} w\right] Z \mathrm{~d} x \\
& =\epsilon^{2} \varrho_{2} f^{\prime} h^{\prime}+\epsilon^{2} \varrho_{2} \varpi f^{\prime} h+O\left(\epsilon^{3}\right),
\end{aligned}
$$

where

$$
\varrho_{2}=2 \int_{\mathbb{R}} w_{x x} Z \mathrm{~d} x .
$$

The estimate of $\mathrm{II}_{7}$ can be proved by the same way as employed in the above estimate.

$$
\mathrm{II}_{7}=\int_{\mathbb{R}} M_{32}(x, z) Z \mathrm{~d} x=\int_{\mathbb{R}}\left(\epsilon^{2} k^{2} \sigma^{-2} f^{2} w_{2}+\epsilon^{3} a_{7}(\epsilon s, \epsilon z)\right) Z \mathrm{~d} x=\epsilon^{3} \mathbf{b}_{1 \epsilon} f^{\prime \prime}+\epsilon^{3} \mathbf{b}_{2 \epsilon} .
$$

Note that $B_{2}(w)=O\left(\epsilon^{3}\right)$, it is easy to check that

$$
\mathrm{II}_{2}=\int_{\mathbb{R}} B_{2}(w) Z \mathrm{~d} x=\epsilon^{3} \mathbf{b}_{1 \epsilon} f^{\prime \prime}+\epsilon^{3} \mathbf{b}_{2 \epsilon} .
$$

Since

$$
\phi_{1}(x, z)=\epsilon a_{11}(\epsilon z) w_{1}(x)+\epsilon a_{12}(\epsilon z)(f(\epsilon z)+h(\epsilon z)) w_{2}(x),
$$

so we obtain

$$
\begin{aligned}
\mathrm{II}_{3} & =\beta^{-2} \int_{\mathbb{R}} \phi_{1, z z} Z \mathrm{~d} x \\
& =\epsilon^{3} \beta^{-2} a_{12}(\epsilon z) f^{\prime \prime} \int_{\mathbb{R}} w_{2}(x) Z(x) \mathrm{d} x+\epsilon^{3} \mathbf{b}_{2 \epsilon} \\
& =\epsilon^{3} \mathbf{b}_{1 \epsilon} f^{\prime \prime}+\epsilon^{3} \mathbf{b}_{2 \epsilon} .
\end{aligned}
$$

Since

$$
\phi_{4}(x, z)=\epsilon^{2} \phi_{41}(x, \epsilon z)+\epsilon^{2} \phi_{42}(x, \epsilon z) \text {, }
$$

it is easy to prove that

$$
\mathrm{II}_{4}=\beta^{-2} \int_{\mathbb{R}} \phi_{4, z z} Z \mathrm{~d} x=O\left(\epsilon^{4}\right) .
$$


According to the expression of $M_{12}(x, z)$ and $M_{22}(x, z)$, we know that the terms in $M_{12}(x, z)$ and $M_{22}(x, z)$ are of order $O\left(\epsilon^{3}\right)$. Hence, it is easy to obtain that

$$
\begin{aligned}
& \mathrm{II}_{5}=\int_{\mathbb{R}} M_{12}(x, z) Z \mathrm{~d} x=O\left(\epsilon^{3}\right), \\
& \mathrm{II}_{6}=\int_{\mathbb{R}} M_{22}(x, z) Z \mathrm{~d} x=O\left(\epsilon^{3}\right) .
\end{aligned}
$$

What's more, we can compute that

$$
\begin{aligned}
\mathrm{II}_{8} & =\int_{\mathbb{R}} M_{42}(x, z) Z(x) \mathrm{d} x \\
& =-2 \epsilon^{2} \beta^{-1}\left(f^{\prime}+\varpi f\right) \xi(\epsilon z) \int_{\mathbb{R}}\left[\epsilon A^{\prime}(\mathfrak{a}(\epsilon z)) \beta Z_{x}+\phi_{22, x z}\right] Z \mathrm{~d} x+O\left(\epsilon^{3}\right) \\
& =O\left(\epsilon^{3}\right) .
\end{aligned}
$$

We need only to compute those parts in $M_{52}(x, z)$ which are even in $x$. It is easy to check that

$$
\mathrm{II}_{9}=\int_{\mathbb{R}} M_{52}(x, z) Z \mathrm{~d} x=-\epsilon^{2} \beta^{-1} k e \int_{\mathbb{R}}\left(Z_{x}+\sigma^{-1} x Z\right) Z \mathrm{~d} x+O\left(\epsilon^{3}\right)=O\left(\epsilon^{3}\right) .
$$

Additionally, we also need to consider some higher order terms in $\mathrm{II}_{9}$. The ones involving first derivative of $e$ are

$$
\begin{aligned}
2 \epsilon^{3} e^{\prime} & \left(\frac{\beta^{\prime}}{\beta^{3}}-\frac{\varpi}{\beta^{2}}\right) \int_{\mathbb{R}} x Z Z_{x} \mathrm{~d} x+\epsilon^{3} e^{\prime}\left(\frac{2 \alpha^{\prime}}{\alpha \beta^{2}}-\frac{\varpi}{\beta^{2}}\right) \int_{\mathbb{R}} Z^{2} \mathrm{~d} x \\
& =\epsilon^{3}\left(\frac{2 \alpha^{\prime}}{\alpha \beta^{2}}-\frac{\beta^{\prime}}{\beta^{3}}\right) e^{\prime} \equiv \epsilon^{3} \hbar_{5}(\epsilon z) e^{\prime},
\end{aligned}
$$

where

$$
\hbar_{5}(\epsilon z)=\frac{2 \alpha^{\prime}(\epsilon z)}{\alpha(\epsilon z) \beta^{2}(\epsilon z)}-\frac{\beta^{\prime}(\epsilon z)}{\beta^{3}(\epsilon z)} .
$$

Moreover, the ones involving second derivative of $e$ in $\mathrm{II}_{9}$ are

$$
\epsilon^{3}\left[\epsilon f \beta^{-2} \hbar_{6}(\epsilon z)+O\left(\epsilon^{2}\right)\right] e^{\prime \prime}(\epsilon z)
$$

with $O\left(\epsilon^{2}\right)$ uniform in $\epsilon$.

In the terms of $\mathrm{II}_{10}$ and $\mathrm{II}_{12}$, we need only to consider those parts which are even in $x$. It is best that the even (in $x$ ) terms in $\mathrm{II}_{10}$ and $\mathrm{II}_{12}$ are of order $o\left(\epsilon^{3}\right)$. Moreover, the terms in $B_{3}\left(\phi_{3}\right)$ are of order $O\left(\epsilon^{3}\right)$. Consequently, we deduce that

$$
\mathrm{II}_{10}+\mathrm{II}_{11}+\mathrm{II}_{12}=\int_{\mathbb{R}} M_{62}(x, z) Z \mathrm{~d} x+\int_{\mathbb{R}} B_{3}\left(\phi_{3}\right) Z \mathrm{~d} x+\int_{\mathbb{R}} B_{3}\left(\phi_{4}\right) Z \mathrm{~d} x=O\left(\epsilon^{3}\right) .
$$

In summary, we have established that, as a function of $\theta=\epsilon z$,

$$
\begin{aligned}
\int_{\mathbb{R}} \mathcal{E} Z \mathrm{~d} x=\epsilon^{3} & {\left[1+\epsilon f \hbar_{6}(\epsilon z)+O\left(\epsilon^{2}\right)\right] \beta^{-2} e^{\prime \prime}+\epsilon^{3} \hbar_{5}(\epsilon z) e^{\prime} } \\
& +\epsilon^{2} \varrho_{2} f^{\prime} h^{\prime}+\epsilon^{2} \varrho_{2} \varpi f^{\prime} h+\epsilon \lambda_{0} e+O\left(\epsilon^{3}\right),
\end{aligned}
$$

where $\hbar_{6}(\epsilon z)$ is a smooth functions of their argument. 


\subsection{Projection of terms involving $\phi$}

We will estimate the terms that involve $\phi$ in (6.1)-(6.4) integrated against the functions $w_{x}$ and $Z$ in the variable $x$. Concerning $w_{x}$, we denote by $\Lambda(\phi)$ the sum of these terms, which can be decomposed as $\Lambda(\phi)=\sum_{i=1}^{3} \Lambda_{i}(\phi)$.

Let $\Lambda_{1}(\epsilon z)=\int_{\mathbb{R}} \chi(\epsilon|x|) B_{3}(\phi) w_{x} \mathrm{~d} x$. We make the following observation: all terms in $B_{3}(\phi)$ carry $\epsilon$ and involve power of $x$ times derivatives of 1,2 orders of $\phi$. The conclusion is that since $w_{x}$ has exponential decay, then

$$
\int_{0}^{1}\left|\Lambda_{1}(\theta)\right|^{2} \mathrm{~d} \theta \leq C \epsilon^{3}\|\phi\|_{H^{2}(\mathcal{S})}^{2} .
$$

Hence,

$$
\left\|\Lambda_{1}\right\|_{L^{2}(0,1)} \leq C \epsilon^{3} .
$$

We shall analyze the properties of the operator $\Lambda_{1}$ acting on the pair $(f, e)$ in $H^{2}(0,1)$. We single out two less regular terms which are operators depending Lipschitz continuously on $(f, e)$. The one whose coefficient depends on $f^{\prime \prime}$ explicitly has the form

$$
\begin{aligned}
\Lambda_{1 *}= & -\epsilon^{2} \beta^{-1} f^{\prime \prime} \int_{\mathbb{R}} \phi_{x} w_{x} \mathrm{~d} x-2 \epsilon^{3} k \beta^{-1} f^{\prime \prime} \int_{\mathbb{R}}\left(\frac{x}{\beta}+f+h\right) \phi_{x} w_{x} \mathrm{~d} x \\
& -\epsilon^{4} \beta^{-1} a_{1}(\epsilon s, \epsilon z) f^{\prime \prime} \int_{\mathbb{R}}\left(\frac{x}{\beta}+f+h\right)^{2} \phi_{x} w_{x} \mathrm{~d} x .
\end{aligned}
$$

Since $\phi$ has Lipschitz dependence on $(f, e)$ in the form (5.21), from Sobolev's embedding, we derive that

$$
\left\|\phi\left(f_{1}, e_{1}\right)-\phi\left(f_{2}, e_{2}\right)\right\|_{L^{\infty}(\mathcal{S})} \leq C \epsilon^{3 / 2}\left[\left\|f_{1}-f_{2}\right\|_{*}+\left\|e_{1}-e_{2}\right\|_{* *}\right] .
$$

Hence,

$$
\left\|\Lambda_{1 *}\left(f_{1}, e_{1}\right)-\Lambda_{1 *}\left(f_{2}, e_{2}\right)\right\|_{L^{2}(0,1)} \leq C^{3+1 / 2}\left[\left\|f_{1}-f_{2}\right\|_{*}+\left\|e_{1}-e_{2}\right\|_{* *}\right] .
$$

Another one comes from second derivative of $\phi$ in $z$

$$
\Lambda_{1 * *}=2 \epsilon k \alpha \int_{\mathbb{R}}\left(\frac{x}{\beta}+f+h\right) \phi_{z z} w_{x} \mathrm{~d} x+\epsilon^{2} a_{1}(\epsilon s, \epsilon z) \alpha \int_{\mathbb{R}}\left(\frac{x}{\beta}+f+h\right)^{2} \phi_{z z} w_{x} \mathrm{~d} x .
$$

Then

$$
\left\|\Lambda_{1 * *}\left(f_{1}, e_{1}\right)-\Lambda_{1 * *}\left(f_{2}, e_{2}\right)\right\|_{L^{2}(0,1)} \leq C \epsilon^{3}\left[\left\|f_{1}-f_{2}\right\|_{*}+\left\|e_{1}-e_{2}\right\|_{* *}\right] .
$$

For fixed $\epsilon$, the remainder $\Lambda_{1}-\Lambda_{1 *}-\Lambda_{1 * *}$ actually defines a compact operator of the pair $(f, e)$ from $H^{2}(0,1)$ into $L^{2}(0,1)$. This is a consequence of the fact that weak convergence in $H^{2}(\mathcal{S})$ implies local strong convergence in $H^{1}(\mathcal{S})$, and the same is the case for $H^{2}(0,1)$ and $C^{1}[0,1]$. If $f_{j}$ and $e_{j}$ are bounded sequences in $H^{2}(0,1)$, then clearly the functions $\phi\left(f_{j}, e_{j}\right)$ constitute a bounded sequence in $H^{2}(\mathcal{S})$. In the above remainder we can integrate by parts once in $x$ if necessary. Averaging against $w_{x}$ which decays exponentially, localizes the situation and the desired result follows.

We also observe that

$$
\Lambda_{2}(\epsilon z)=\int_{\mathbb{R}} \eta_{\delta}^{\epsilon}(s) \mathcal{N}(\phi) w_{x} \mathrm{~d} x
$$


can be estimated similarly. Using the definition of $\mathcal{N}(\phi)$ and the exponential decay of $w_{x}$ we get

$$
\left\|\Lambda_{2}\right\|_{L^{2}(0,1)} \leq C \epsilon^{1 / 2}\|\phi\|_{H^{2}(\mathcal{S})} \leq C \epsilon^{3} .
$$

Finally, let us consider

$$
\Lambda_{3}(\epsilon z)=\int_{\mathbb{R}} p\left(\beta^{-2} \chi(\epsilon|x|) \mathbf{W}^{p-1}-w^{p-1}\right) \phi w_{x} \mathrm{~d} x .
$$

Since

$$
v_{5}=w+\phi_{1}+\phi_{2}+\epsilon e Z+\phi_{3}+\phi_{4} \quad \text { and } \quad \phi_{1}+\phi_{2}+\epsilon e Z+\phi_{3}+\phi_{4}
$$

can be estimated as

$$
\left|\phi_{1}\right|+\left|\phi_{2}\right|+\epsilon|e Z|+\left|\phi_{3}\right|+\left|\phi_{4}\right| \leq C \epsilon\left(1+|x|^{2}\right) e^{-|x|},
$$

we obtain that for some $\rho>0$ the following uniform bound holds

$$
\left|\left(\beta^{-2} \chi(\epsilon|x|) \mathbf{W}^{p-1}-w^{p-1}\right) w_{x}\right| \leq C \epsilon e^{-\rho|x|} .
$$

From here we find that

$$
\left\|\Lambda_{3}\right\|_{L^{2}(0,1)} \leq C \epsilon^{3 / 2}\|\phi\|_{H^{2}(\mathcal{S})} \leq C \epsilon^{3} .
$$

These two terms $\Lambda_{2}$ and $\Lambda_{3}$ also define compact operators similarly as before.

We observe that exactly the same estimates can be carried out in the terms obtained from integration against $Z$.

\subsection{Projection of errors on the boundary}

In this section we compute the projection of errors on the boundary. Without loss of generality, only the projections of the error components on $\partial_{0} \mathcal{S}$ will be given.

The main errors on the boundary integrated against $w_{x}$ and $Z$ in the variable $x$ can be computed as the following:

$$
\begin{aligned}
& \int_{\mathbb{R}} g_{0}(x) w_{x} \mathrm{~d} x \\
& =-\epsilon \beta\left(k_{1} f+f^{\prime}\right) \int_{\mathbb{R}} w_{x}^{2} \mathrm{~d} x-\epsilon^{2} b_{5} a_{11} \int_{\mathbb{R}} x w_{1, x} w_{x} \mathrm{~d} x \\
& \quad-\epsilon^{2} \beta\left(k_{1} f+f^{\prime}\right) \int_{\mathbb{R}}\left\{a_{12}(f+h) w_{2, x}+e Z_{x}+\beta^{-1}\left[A(0) Z_{x}+\phi_{22}(x, 0)\right]\right\} w_{x} \mathrm{~d} x \\
& \quad-\epsilon^{2} \int_{\mathbb{R}}\left\{b_{1}\left(\beta^{-2} x^{2}+h^{2}+2 f h\right) \beta-k\left[\beta^{-2} \beta^{\prime} x^{2}-\left(f h^{\prime}+h f^{\prime}+h h^{\prime}\right) \beta\right]\right\} w_{x}^{2} \mathrm{~d} x \\
& \quad+\epsilon^{2}\left(\alpha^{\prime} \alpha^{-1} a_{11}+a_{11}^{\prime}\right) \int_{\mathbb{R}} w_{1} w_{x} \mathrm{~d} x+\epsilon^{2} k \alpha^{\prime} \alpha^{-1} \beta^{-1} \int_{\mathbb{R}} x w w_{x} \mathrm{~d} x \\
& \quad+\epsilon^{2} k \beta^{-2} \int_{\mathbb{R}}\left[\epsilon A^{\prime}(0) \beta(0) Z(x)+\phi_{22, z}(x, 0)\right] x w_{x} \mathrm{~d} x+O\left(\epsilon^{3}\right) .
\end{aligned}
$$

Using the following formulas

$$
\int_{\mathbb{R}} Z^{2}(x) \mathrm{d} x=-2 \int_{\mathbb{R}} x Z_{x}(x) Z(x) \mathrm{d} x=1,
$$


we get the following two estimates

$$
\int_{\mathbb{R}} g_{0}(x) Z(x) \mathrm{d} x=\frac{\epsilon^{2}}{2} b_{5} e(0)+\epsilon^{2}\left[\alpha^{\prime}(0) \alpha^{-1}(0) e(0)+e^{\prime}(0)\right]+O\left(\epsilon^{3}\right) .
$$

Higher order errors can be proceeded as follows:

$$
\begin{aligned}
\int_{\mathbb{R}} & D_{3}^{0}(\phi(x, 0)) w_{x} \mathrm{~d} x \\
= & \epsilon \int_{\mathbb{R}}\left[b_{5} x+\beta\left(k_{1} f+f^{\prime}\right)\right] \phi_{x}(x, 0) w_{x} \mathrm{~d} x \\
& -\epsilon \alpha^{\prime} \alpha^{-1} \int_{\mathbb{R}} \phi(x, 0) w_{x} \mathrm{~d} x-\epsilon k \int_{\mathbb{R}}\left(\frac{x}{\beta}+f+h\right) \phi_{z}(x, 0) w_{x} \mathrm{~d} x \\
& +\epsilon^{2} \int_{\mathbb{R}}\left[b_{1}\left(\frac{x}{\beta}+f+h\right)^{2} \beta-k\left(\frac{x}{\beta}+f+h\right)\left(\frac{\beta^{\prime}}{\beta} x-\beta f^{\prime}-\beta h^{\prime}\right)\right] \phi_{x}(x, 0) w_{x} \mathrm{~d} x \\
& -\epsilon^{2} k \frac{\alpha^{\prime}}{\alpha} \int_{\mathbb{R}}\left(\frac{x}{\beta}+f+h\right) \phi(x, 0) w_{x} \mathrm{~d} x+\epsilon^{2} b_{2} \int_{\mathbb{R}}\left(\frac{x}{\beta}+f+h\right)^{2} \phi_{z}(x, 0) w_{x} \mathrm{~d} x \\
= & O\left(\epsilon^{\frac{5}{2}}\right),
\end{aligned}
$$

and also

$$
\begin{aligned}
& \int_{\mathbb{R}} D_{3}^{0}(\phi(x, 0)) Z \mathrm{~d} x \\
& =\epsilon \int_{\mathbb{R}}\left[b_{5} x+\beta\left(k_{1} f+f^{\prime}\right)\right] \phi_{x}(x, 0) Z \mathrm{~d} x \\
& \quad-\epsilon \alpha^{\prime} \alpha^{-1} \int_{\mathbb{R}} \phi(x, 0) Z \mathrm{~d} x-\epsilon k \int_{\mathbb{R}}\left(\frac{x}{\beta}+f+h\right) \phi_{z}(x, 0) Z \mathrm{~d} x \\
& \quad+\epsilon^{2} \int_{\mathbb{R}}\left[b_{1}\left(\frac{x}{\beta}+f+h\right)^{2} \beta-k\left(\frac{x}{\beta}+f+h\right)\left(\frac{\beta^{\prime}}{\beta} x-\beta f^{\prime}-\beta h^{\prime}\right)\right] \phi_{x}(x, 0) Z \mathrm{~d} x \\
& \quad-\epsilon^{2} k \frac{\alpha^{\prime}}{\alpha} \int_{\mathbb{R}}\left(\frac{x}{\beta}+f+h\right) \phi(x, 0) Z \mathrm{~d} x+\epsilon^{2} b_{2} \int_{\mathbb{R}}\left(\frac{x}{\beta}+f+h\right)^{2} \phi_{z}(x, 0) Z \mathrm{~d} x \\
& =O\left(\epsilon^{\frac{5}{2}}\right) .
\end{aligned}
$$

The term $D_{0}^{0}(\phi)$ on the boundary integrated against $w_{x}$ and $Z$ in the variable $x$ are of size of order $O\left(\epsilon^{3}\right)$.

\section{The system for $(f, e):$ proof of the theorem}

Using the estimates in previous section and $\theta=\epsilon z$, and then defining the operators

$$
\begin{aligned}
& \mathbb{L}_{1}(f) \equiv f^{\prime \prime}+\left[\hbar_{1}(\theta)+\alpha_{1}(\theta / \epsilon)\right] f^{\prime}+\left[\hbar_{2}(\theta)+\alpha_{2}(\theta / \epsilon)\right] f, \\
& \mathbb{L}_{2}(e) \equiv \epsilon^{2}\left(\beta^{-2} e^{\prime \prime}+\hbar_{5}(\theta) e^{\prime}\right)+\lambda_{0} e,
\end{aligned}
$$

where $\hbar_{1}(\theta), \hbar_{2}(\theta), \alpha_{1}(z)$ and $\alpha_{2}(z)$ are smooth functions defined in (4.67), (4.68), (4.59) and (4.60), we find the following nonlinear system of differential equations for the parameters $(f, e)$

$$
\mathbb{L}_{1}(f)=\hbar_{3}(\theta) e+\epsilon^{2} \hbar_{4}(\theta) e^{\prime \prime}+\epsilon \mathcal{M}_{1, \epsilon},
$$




$$
\mathbb{L}_{2}(e)=\epsilon^{3} f \hbar_{6}(\theta) e^{\prime \prime}+\epsilon \varrho_{2} f^{\prime} h^{\prime}+\epsilon \varrho_{2} \varpi f^{\prime} h+\epsilon^{2} \mathcal{M}_{2, \epsilon},
$$

with the boundary conditions

$$
\begin{aligned}
& f^{\prime}(1)+k_{2} f(1)+\mathcal{M}_{1}^{1}(f, e)=0, \\
& f^{\prime}(0)+k_{1} f(0)+\mathcal{M}_{1}^{2}(f, e)=0, \\
& e^{\prime}(1)+\tilde{b}_{6} e(1)+\mathcal{M}_{2}^{1}(f, e)=0, \\
& e^{\prime}(0)+\tilde{b}_{5} e(0)+\mathcal{M}_{2}^{2}(f, e)=0 .
\end{aligned}
$$

The constants $\tilde{b}_{6}$ and $\tilde{b}_{5}$ are given by

$$
\tilde{b}_{6}=\frac{b_{6}}{2}+\frac{\alpha^{\prime}(1)}{\alpha(1)}, \quad \tilde{b}_{5}=\frac{b_{5}}{2}+\frac{\alpha^{\prime}(0)}{\alpha(0)},
$$

where $b_{5}$ and $b_{6}$ are constants defined in (3.33) and (3.37). $\mathcal{M}_{j}^{i}$ 's are some terms of order $O\left(\epsilon^{1 / 2}\right)$. The operators $\mathcal{M}_{1, \epsilon}$ and $\mathcal{M}_{2, \epsilon}$ can be decomposed in the following forms

$$
\mathcal{M}_{l, \epsilon}(f, e)=\mathbf{A}_{l, \epsilon}(f, e)+\mathbf{K}_{l, \epsilon}(f, e), \quad l=1,2
$$

where $\mathbf{K}_{l, \epsilon}$ 's are uniformly bounded in $L^{2}(0,1)$ for $(f, e)$ in $\mathcal{F}$ and are also compact. The operators $\mathbf{A}_{l, \epsilon}, i=1,2$, are Lipschitz in this region, see (6.33)-(6.34)

$$
\left\|\mathbf{A}_{l, \epsilon}\left(f_{1}, e_{1}\right)-\mathbf{A}_{l, \epsilon}\left(f_{2}, e_{2}\right)\right\|_{L^{2}(0,1)} \leq C\left[\left\|f_{1}-f_{2}\right\|_{*}+\left\|e_{1}-e_{2}\right\|_{* *}\right] .
$$

Before solving (7.3)-(7.8), some basic facts about the invertibility of corresponding linear operators will be derived. Firstly, we consider the following problem

$$
\begin{array}{cc}
f^{\prime \prime}(\theta)+\left(\hbar_{1}(\theta)+\alpha_{1}(\theta / \epsilon)\right) f^{\prime}(\theta)+\left(\hbar_{2}(\theta)+\alpha_{2}(\theta / \epsilon)\right) f(\theta)=\mathbf{g}(\theta), \\
f^{\prime}(0)+k_{1} f(0)=0, & f^{\prime}(1)+k_{2} f(1)=0 .
\end{array}
$$

Lemma 7.1. (1). Under the non-degenerate condition (2.11), if $\mathbf{g} \in L^{2}(0,1)$, then there is a constant $\epsilon_{0}$ for each $0<\epsilon<\epsilon_{0}$ satisfying (1.14), such that problem (7.10) has a unique solution $f \in H^{2}(0,1)$, which satisfies

$$
\|f\|_{*} \leq C\|\mathbf{g}\|_{L^{2}(0,1)}
$$

(2). Moreover, if

$$
\mathbf{g}(\theta)=G_{1}(\theta / \epsilon)+G_{2}(\theta / \epsilon)+G_{3}(\theta / \epsilon),
$$

where $G_{1}, G_{2}$ and $G_{3}$ are given in (4.61), (4.62) and (4.64), then

$$
\|f\|_{*} \leq C \epsilon^{\frac{1}{2}} .
$$

Proof. (1). Under the non-degeneracy condition (2.11), the existence part comes from the a priori estimate and the continuity method. Hence, we focus on the derivations of the estimates.

First of all, by the relation

$$
\vartheta=\frac{\mathfrak{a}(\theta)}{\ell},
$$


where $\ell$ and $a(\theta)$ are given in (4.23) and (4.27), we make the following transformation:

$$
\begin{array}{clrl}
\eta(\vartheta)=f(\theta), & \hat{\beta}(\vartheta)=\beta(\theta), & \hat{\mathbf{g}}(\vartheta)=\mathbf{g}(\theta), \\
\hat{\hbar}_{1}(\vartheta)=\hbar_{1}(\theta), & \hat{\hbar}_{2}(\vartheta)=\hbar_{2}(\theta), & \hat{\varpi}(\vartheta)=\varpi(\theta) .
\end{array}
$$

Then (7.10) gets transformed into

$$
\begin{gathered}
\eta_{\vartheta \vartheta}+\frac{\hat{\beta}^{\prime}(\vartheta)}{\hat{\beta}(\vartheta)} \eta_{\vartheta}+\frac{\hat{\hbar}_{1}(\vartheta) \ell}{\hat{\beta}(\vartheta)} \eta_{\vartheta}+\frac{\hat{\hbar}_{2}(\vartheta) \ell^{2}}{\hat{\beta}^{2}(\vartheta)} \eta+\frac{\hat{\alpha}_{1}(\vartheta) \ell}{\hat{\beta}(\vartheta)} \eta_{\vartheta}+\frac{\hat{\alpha}_{2}(\vartheta) \ell^{2}}{\hat{\beta}^{2}(\vartheta)} \eta=\frac{\ell^{2}}{\hat{\beta}^{2}(\vartheta)} \hat{\mathbf{g}}(\vartheta), \\
\eta^{\prime}(1)+k_{2} \eta(1)=0, \quad \eta^{\prime}(0)+k_{1} \eta(0)=0 .
\end{gathered}
$$

Here the terms $\hat{\alpha}_{1}$ and $\hat{\alpha}_{2}$ are define as

$$
\hat{\alpha}_{1}(\vartheta)=\hat{\bar{\alpha}}_{1}(\vartheta)+\hat{\tilde{\alpha}}_{1}(\vartheta), \quad \hat{\alpha}_{2}(\vartheta)=\hat{\bar{\alpha}}_{2}(\vartheta)+\hat{\tilde{\alpha}}_{2}(\vartheta),
$$

where we have denoted

$$
\begin{gathered}
\hat{\bar{\alpha}}_{2}(\vartheta)=\hat{\varpi}(\vartheta) \hat{\bar{\alpha}}_{1}(\vartheta), \quad \hat{\tilde{\alpha}}_{2}(\vartheta)=\hat{\varpi}(\vartheta) \hat{\tilde{\alpha}}_{1}(\vartheta), \\
\hat{\bar{\alpha}}_{1}(\vartheta)=2 \varrho_{1}^{-1} \epsilon \hat{\chi}(\vartheta) A^{\prime}(\ell \vartheta) \hat{\beta}(\vartheta) \int_{\mathbb{R}} Z_{x} w_{x} \mathrm{~d} x, \\
\hat{\tilde{\alpha}}_{1}(\vartheta)=2 \varrho_{1}^{-1} \hat{\chi}(\vartheta) \int_{\mathbb{R}} \phi_{22, x z}\left(x, \frac{\mathfrak{a}^{-1}(\ell \vartheta)}{\epsilon}\right) w_{x} \mathrm{~d} x,
\end{gathered}
$$

and

$$
\hat{\chi}(\vartheta) \equiv \frac{\hat{\chi}_{0}(\vartheta)}{\beta(0)}+\frac{1-\hat{\chi}_{0}(\vartheta)}{\beta(1)} .
$$

In the above, $\mathfrak{a}^{-1}$ is the inverse of the map $\mathfrak{a}$ given in (4.27).

Define the operator $\mathbb{L}_{0}$ in the form

$$
\mathbb{L}_{0}(\eta):=\eta^{\prime \prime}(\vartheta)+\mathfrak{q}_{1}(\vartheta) \eta^{\prime}(\vartheta)+\mathfrak{q}_{2}(\vartheta) \eta,
$$

where

$$
\mathfrak{q}_{1}(\vartheta)=\frac{\hat{\beta}^{\prime}(\vartheta)}{\hat{\beta}(\vartheta)}+\frac{\hat{\hbar}_{1}(\vartheta) \ell}{\hat{\beta}(\vartheta)}, \quad \mathfrak{q}_{2}(\vartheta)=\frac{\hat{\hbar}_{2}(\vartheta) \ell^{2}}{\hat{\beta}^{2}(\vartheta)} .
$$

There exists an orthonormal basis of $L^{2}(0,1)$ constituted by eigenfunctions $\left\{y_{j}\right\}$, associated to the eigenvalues $\left\{\lambda_{j}\right\}$, of the following eigenvalue problem

$$
\begin{aligned}
-\mathbb{L}_{0}(y(\vartheta)) & =\lambda y(\vartheta), \quad 0<\vartheta<1, \\
y^{\prime}(1)+k_{2} y(1) & =0, \quad y^{\prime}(0)+k_{1} y(0)=0 .
\end{aligned}
$$

The result in [39] shows that, as $j \rightarrow \infty$,

$$
\sqrt{\lambda_{j}}=j \pi+\frac{k_{2}-k_{1}}{j \pi}+O\left(\frac{1}{j^{3}}\right) .
$$

It is easy to see that there exists a positive constant $C$ such that $\left|y_{j}^{\prime}(\vartheta)\right| \leq C j$ for all $j \in \mathbb{N}$. 
We then expand

$$
\begin{aligned}
\frac{\ell^{2}}{\hat{\beta}^{2}(\vartheta)} \hat{\mathbf{g}}(\vartheta) & =\sum_{j=0}^{\infty} \mathbf{g}_{j} y_{j}(\vartheta), & \eta(\vartheta) & =\sum_{j=0}^{\infty} a_{j} y_{j}(\vartheta), \\
\frac{\hat{\bar{\alpha}}_{1} \ell}{\hat{\beta}(\vartheta)} \eta^{\prime}(\vartheta) & =\sum_{j=0}^{\infty} \bar{d}_{j} y_{j}(\vartheta), & \frac{\hat{\bar{\alpha}}_{2} \ell^{2}}{\hat{\beta}^{2}(\vartheta)} \eta(\vartheta) & =\sum_{j=0}^{\infty} \bar{c}_{j} y_{j}(\vartheta), \\
\frac{\hat{\tilde{\alpha}}_{1} \ell}{\hat{\beta}(\vartheta)} \eta^{\prime}(\vartheta) & =\sum_{j=0}^{\infty} \tilde{d}_{j} y_{j}(\vartheta), & \frac{\hat{\tilde{\alpha}}_{2} \ell^{2}}{\hat{\beta}^{2}(\vartheta)} \eta(\vartheta) & =\sum_{j=0}^{\infty} \tilde{c}_{j} y_{j}(\vartheta), \\
\frac{\hat{\alpha}_{1} \ell}{\hat{\beta}(\vartheta)} \eta^{\prime}(\vartheta) & =\sum_{j=0}^{\infty} d_{j} y_{j}(\vartheta), & \frac{\hat{\alpha}_{2} \ell^{2}}{\hat{\beta}^{2}(\vartheta)} \eta(\vartheta) & =\sum_{j=0}^{\infty} c_{j} y_{j}(\vartheta) .
\end{aligned}
$$

Now, we will estimate the Fourier coefficients above by using the asymptotic behaviors of the following two terms

$$
\Phi_{j, 1}(\vartheta)=\int_{0}^{\vartheta} \sin \left(\frac{\sqrt{\lambda_{0}}}{\epsilon} \ell s\right) y_{j}(s) \mathrm{d} s, \quad \Phi_{j, 2}(\vartheta)=\int_{0}^{\vartheta} \cos \left(\frac{\sqrt{\lambda_{0}}}{\epsilon} \ell s\right) y_{j}(s) \mathrm{d} s .
$$

In the following formula, by using the equation for $y_{j}$ and integrating by parts two times

$$
\begin{aligned}
\Phi_{j, 1}(\vartheta)=- & \frac{\epsilon^{2}}{\lambda_{0} \ell^{2}} \int_{0}^{\vartheta}\left[\sin \left(\frac{\sqrt{\lambda_{0}}}{\epsilon} \ell s\right)\right]^{\prime \prime} y_{j}(s) \mathrm{d} s \\
=- & \frac{\epsilon}{\sqrt{\lambda_{0}} \ell}\left[\cos \left(\frac{\sqrt{\lambda_{0}}}{\epsilon} \ell \vartheta\right) y_{j}(\vartheta)-y_{j}(0)\right]+\frac{\epsilon^{2}}{\lambda_{0} \ell^{2}} \sin \left(\frac{\sqrt{\lambda_{0}}}{\epsilon} \ell \vartheta\right) y_{j}^{\prime}(\vartheta) \\
& -\frac{\epsilon^{2}}{\lambda_{0} \ell^{2}} \int_{0}^{\vartheta} \sin \left(\frac{\sqrt{\lambda_{0}}}{\epsilon} \ell s\right) y_{j}^{\prime \prime}(s) \mathrm{d} s \\
=- & \frac{\epsilon}{\sqrt{\lambda_{0}} \ell}\left[\cos \left(\frac{\sqrt{\lambda_{0}}}{\epsilon} \ell \vartheta\right) y_{j}(\vartheta)-y_{j}(0)\right]+\frac{\epsilon^{2}}{\lambda_{0} \ell^{2}} \sin \left(\frac{\sqrt{\lambda_{0}}}{\epsilon} \ell \vartheta\right) y_{j}^{\prime}(\vartheta) \\
& -\frac{\epsilon^{2}}{\lambda_{0} \ell^{2}} \int_{0}^{\vartheta} \sin \left(\frac{\sqrt{\lambda_{0}}}{\epsilon} \ell s\right)\left[y_{j}^{\prime \prime}(s)+\mathfrak{q}_{1}(s) y_{j}^{\prime}(s)+\mathfrak{q}_{2}(s) y_{j}(s)\right] \mathrm{d} s \\
& +\frac{\epsilon^{2}}{\lambda_{0} \ell^{2}} \int_{0}^{\vartheta} \sin \left(\frac{\sqrt{\lambda_{0}}}{\epsilon} \ell s\right)\left[\mathfrak{q}_{1}(s) y_{j}^{\prime}(s)+\mathfrak{q}_{2}(s) y_{j}(s)\right] \mathrm{d} s \\
= & \frac{\epsilon^{2}}{\lambda_{0} \ell^{2}} \int_{0}^{\vartheta} \sin \left(\frac{\sqrt{\lambda_{0}}}{\epsilon} \ell s\right) \lambda_{j} y_{j}(s) \mathrm{d} s-\frac{\epsilon}{\sqrt{\lambda_{0} \ell}}\left[\cos \left(\frac{\sqrt{\lambda_{0}}}{\epsilon} \ell \vartheta\right) y_{j}(\vartheta)-y_{j}(0)\right] \\
& +\frac{\epsilon^{2}}{\lambda_{0} \ell^{2}} \sin \left(\frac{\sqrt{\lambda_{0}}}{\epsilon} \ell \vartheta\right) y_{j}^{\prime}(\vartheta)+\frac{\epsilon^{2}}{\lambda_{0} \ell^{2}} \int_{0}^{\vartheta} \sin \left(\frac{\sqrt{\lambda_{0}}}{\epsilon} \ell s\right)\left[\mathfrak{q}_{1}(s) y_{j}^{\prime}(s)+\mathfrak{q}_{2}(s) y_{j}(s)\right] \mathrm{d} s,
\end{aligned}
$$

we can get

$$
\begin{aligned}
\left(1-\frac{\epsilon^{2} \lambda_{j}}{\lambda_{0} \ell^{2}}\right) \Phi_{j, 1}(\vartheta)=- & \frac{\epsilon}{\sqrt{\lambda_{0} \ell}}\left[\cos \left(\frac{\sqrt{\lambda_{0}}}{\epsilon} \ell \vartheta\right) y_{j}(\vartheta)-y_{j}(0)\right]+\frac{\epsilon^{2}}{\lambda_{0} \ell^{2}} \sin \left(\frac{\sqrt{\lambda_{0}}}{\epsilon} \ell \vartheta\right) y_{j}^{\prime}(\vartheta) \\
& +\frac{\epsilon^{2}}{\lambda_{0} \ell^{2}} \int_{0}^{\vartheta} \sin \left(\frac{\sqrt{\lambda_{0}}}{\epsilon} \ell s\right)\left[\mathfrak{q}_{1}(s) y_{j}^{\prime}(s)+\mathfrak{q}_{2}(s) y_{j}(s)\right] \mathrm{d} s .
\end{aligned}
$$

This implies that

$$
\left|\Phi_{j, 1}(\vartheta)\right| \leq \frac{C \epsilon(\epsilon j+1)}{\left|\lambda_{0} \ell^{2}-\epsilon^{2} \lambda_{j}\right|}
$$


Similarly, it can be derived

$$
\left|\Phi_{j, 2}(\vartheta)\right| \leq \frac{C \epsilon(\epsilon j+1)}{\left|\lambda_{0} \ell^{2}-\epsilon^{2} \lambda_{j}\right|}
$$

By using the gap condition (1.14), we can write $\hat{\bar{\alpha}}_{2}$ in the form

$$
\hat{\bar{\alpha}}_{2}=\bar{K}_{1} \hat{\varpi}(\vartheta) \hat{\chi}(\vartheta) \hat{\beta}(\vartheta) \sin \left(\frac{\sqrt{\lambda_{0}}}{\epsilon} \ell \vartheta\right)+\bar{K}_{2} \hat{\varpi}(\vartheta) \hat{\chi}(\vartheta) \hat{\beta}(\vartheta) \cos \left(\frac{\sqrt{\lambda_{0}}}{\epsilon} \ell \vartheta\right),
$$

for parameters $\bar{K}_{1}, \bar{K}_{2}$ depending on $\epsilon$, which are also bounded by a universal constant independent of $\epsilon$. Hence,

$$
\begin{aligned}
\bar{c}_{j} & =\int_{0}^{1}\left[\bar{K}_{1} \sin \left(\frac{\sqrt{\lambda_{0}}}{\epsilon} \ell \vartheta\right)+\bar{K}_{2} \cos \left(\frac{\sqrt{\lambda_{0}}}{\epsilon} \ell \vartheta\right)\right] \hat{\varpi}(\vartheta) \hat{\chi}(\vartheta) \frac{\ell^{2}}{\hat{\beta}(\vartheta)} \eta(\vartheta) y_{j}(\vartheta) \mathrm{d} \vartheta \\
& =\bar{K}_{1} \ell^{2} \int_{0}^{1} \hat{\varpi}(\vartheta) \hat{\chi}(\vartheta) \hat{\beta}^{-1}(\vartheta) \Phi_{j, 1}^{\prime}(\vartheta) \eta(\vartheta) \mathrm{d} \vartheta+\bar{K}_{2} \ell^{2} \int_{0}^{1} \hat{\varpi}(\vartheta) \hat{\chi}(\vartheta) \hat{\beta}^{-1}(\vartheta) \Phi_{j, 2}^{\prime}(\vartheta) \eta(\vartheta) \mathrm{d} \vartheta .
\end{aligned}
$$

Integrating once by parts and using (7.14)-(7.15), we obtain

$$
\left|\bar{c}_{j}\right| \leq \frac{C \epsilon(\epsilon j+1)}{\left|\lambda_{0} \ell^{2}-\epsilon^{2} \lambda_{j}\right|}\left\{\left\|\eta^{\prime}\right\|_{L^{2}}+\|\eta\|_{L^{2}}\right\} .
$$

A similar approach will imply

$$
\left|\bar{d}_{j}\right| \leq \frac{C \epsilon(\epsilon j+1)}{\left|\lambda_{0} \ell^{2}-\epsilon^{2} \lambda_{j}\right|}\left\{\left\|\eta^{\prime}\right\|_{L^{2}}+\|\eta\|_{L^{2}}\right\} .
$$

The estimate of $\tilde{d}_{j}$ can be showed as

$$
\begin{aligned}
\left|\tilde{d}_{j}\right| & =\left|\int_{0}^{1} \frac{\hat{\tilde{\alpha}}_{1} \ell}{\hat{\beta}(\vartheta)} \eta^{\prime}(\vartheta) y_{j}(\vartheta) \mathrm{d} \vartheta\right| \\
& =2 \varrho_{1}^{-1} \ell\left|\int_{0}^{1} \int_{\mathbb{R}} \hat{\chi}(\vartheta) \hat{\beta}^{-1}(\vartheta) \eta^{\prime}(\vartheta) \phi_{22, x z}\left(x, \frac{\mathfrak{a}^{-1}(\ell \vartheta)}{\epsilon}\right) w_{x} y_{j}(\vartheta) \mathrm{d} x \mathrm{~d} \vartheta\right| \\
& \leq C\left[\int_{0}^{1} \int_{\mathbb{R}}\left|\hat{\chi}(\vartheta) \hat{\beta}^{-1}(\vartheta) \eta^{\prime}(\vartheta) y_{j}(\vartheta) w_{x}\right|^{2} \mathrm{~d} x \mathrm{~d} \vartheta\right]^{\frac{1}{2}}\left[\int_{0}^{1} \int_{\mathbb{R}}\left|\phi_{22, x z}\left(x, \frac{\mathfrak{a}^{-1}(\ell \vartheta)}{\epsilon}\right)\right|^{2} \mathrm{~d} x \mathrm{~d} \vartheta\right]^{\frac{1}{2}} \\
& \leq C \epsilon^{\frac{1}{2}}\left\|\eta^{\prime}\right\|_{L^{2}},
\end{aligned}
$$

where $\mathfrak{a}^{-1}$ is the inverse of the map $\mathfrak{a}$ given in (4.27). Similar estimates hold for $\tilde{c}_{j}$

$$
\left|\tilde{c}_{j}\right| \leq C \epsilon^{\frac{1}{2}}\|\eta\|_{L^{2}} .
$$

Whence, from the equations

$$
\begin{array}{r}
-\lambda_{j} a_{j}+c_{j}+d_{j}=\mathbf{g}_{j}, \\
\bar{c}_{j}+\tilde{c}_{j}=c_{j}, \quad \bar{d}_{j}+\tilde{d}_{j}=d_{j},
\end{array}
$$


and estimates of the Fourier coefficients $\bar{c}_{j}, \bar{d}_{j}, \tilde{c}_{j}$ and $\tilde{d}_{j}$, we get

$$
\left|a_{j}\right| \leq\left|\frac{\mathbf{g}_{j}}{\lambda_{j}}\right|+\frac{C \epsilon(\epsilon j+1)}{\left|\lambda_{j}\left(\lambda_{0} \ell^{2}-\epsilon^{2} \lambda_{j}\right)\right|} \times\left\{\left\|\eta^{\prime}\right\|_{L^{2}}+\|\eta\|_{L^{2}}\right\}+\frac{C \epsilon^{\frac{1}{2}}}{\lambda_{j}} \times\left\{\left\|\eta^{\prime}\right\|_{L^{2}}+\|\eta\|_{L^{2}}\right\} .
$$

Moreover, from the asymptotic expression of $\lambda_{j}$ in (7.13), it can be shown

$$
\left\|\eta^{\prime}\right\|_{L^{2}}^{2}+\|\eta\|_{L^{2}}^{2} \leq C\|\mathbf{g}\|_{L^{2}}^{2}+C_{*} \sum_{j} \frac{j^{2} \epsilon^{2}(\epsilon j+1)^{2}}{\left|\lambda_{j}\right|^{2}\left(\lambda_{0} \ell^{2}-\epsilon^{2} \lambda_{j}\right)^{2}} \times\left\{\left\|\eta^{\prime}\right\|_{L^{2}}^{2}+\|\eta\|_{L^{2}}^{2}\right\},
$$

where the positive constant $C_{*}$ does not depend on $\epsilon$. From the asymptotic expression of $\lambda_{j}$, there exists a positive constant $\epsilon_{0}$ such that, for all positive $\epsilon<\epsilon_{0}$ satisfying (1.14), elementary analysis will imply the following estimates

$$
\begin{aligned}
& \sum_{2 \epsilon^{2} \lambda_{j} \geq 3 \lambda_{0} \ell^{2}} \frac{j^{2} \epsilon^{2}(\epsilon j+1)^{2}}{\left|\lambda_{j}\right|^{2}\left(\lambda_{0} \ell^{2}-\epsilon^{2} \lambda_{j}\right)^{2}} \leq C \epsilon^{2}, \\
& \sum_{\lambda_{0} \ell^{2}<2 \epsilon^{2} \lambda_{j}<3 \lambda_{0} \ell^{2}} \frac{j^{2} \epsilon^{2}(\epsilon j+1)^{2}}{\left|\lambda_{j}\right|^{2}\left(\lambda_{0} \ell^{2}-\epsilon^{2} \lambda_{j}\right)^{2}} \leq C \epsilon, \\
& \sum_{2 \epsilon^{2} \lambda_{j} \leq \lambda_{0} \ell^{2}} \frac{j^{2} \epsilon^{2}(\epsilon j+1)^{2}}{\left|\lambda_{j}\right|^{2}\left(\lambda_{0} \ell^{2}-\epsilon^{2} \lambda_{j}\right)^{2}} \leq C \epsilon^{2} .
\end{aligned}
$$

Hence, we can prove that

$$
\left\|\eta^{\prime}\right\|_{L^{2}}^{2}+\|\eta\|_{L^{2}}^{2} \leq C\|\mathbf{g}\|_{L^{2}}^{2}
$$

Since

$$
\left\|\eta^{\prime \prime}\right\|_{L^{2}} \leq C\left(\|\mathbf{g}\|_{L^{2}}+\left\|\eta^{\prime}\right\|_{L^{2}}+\|\eta\|_{L^{2}}^{2}\right)
$$

the final result then follows easily.

(2). If $\mathbf{g}(\theta)=G_{1}(\theta / \epsilon)+G_{2}(\theta / \epsilon)+G_{3}(\theta / \epsilon)$, using the same argument as the proof of formulas (7.16) and (7.17), we can easily carry out the estimate of $\mathbf{g}_{j}$ in the form

$$
\left|\mathbf{g}_{j}\right| \leq \frac{C \epsilon(\epsilon j+1)}{\left|\lambda_{0} \ell^{2}-\epsilon^{2} \lambda_{j}\right|}+C \epsilon^{\frac{1}{2}} .
$$

For all positive $\epsilon<\epsilon_{0}$ satisfying (1.14), a similar analysis will imply the following estimates

$$
\begin{aligned}
\sum_{2 \epsilon^{2} \lambda_{j} \geq 3 \lambda_{0} \ell^{2}} \frac{\epsilon(\epsilon j+1)}{\left|\lambda_{j}\left(\lambda_{0} \ell^{2}-\epsilon^{2} \lambda_{j}\right)\right|} & \leq C \epsilon^{\frac{1}{2}}, \\
\sum_{\lambda_{0} \ell^{2}<2 \epsilon^{2} \lambda_{j}<3 \lambda_{0} \ell^{2}} \frac{\epsilon(\epsilon j+1)}{\left|\lambda_{j}\left(\lambda_{0} \ell^{2}-\epsilon^{2} \lambda_{j}\right)\right|} & \leq C \epsilon^{\frac{1}{2}}, \\
\sum_{2 \epsilon^{2} \lambda_{j} \leq \lambda_{0} \ell^{2}} \frac{\epsilon(\epsilon j+1)}{\left|\lambda_{j}\left(\lambda_{0} \ell^{2}-\epsilon^{2} \lambda_{j}\right)\right|} & \leq C \epsilon^{\frac{1}{2}} .
\end{aligned}
$$

Therefore, from (7.18) and the estimate of $\mathbf{g}_{j}$, we get

$$
\left\|\eta^{\prime}\right\|_{L^{2}}^{2}+\|\eta\|_{L^{2}}^{2} \leq C \epsilon+C_{*} \sum_{j} \frac{j^{2} \epsilon^{2}(\epsilon j+1)^{2}}{\left|\lambda_{j}\right|^{2}\left(\lambda_{0} \ell^{2}-\epsilon^{2} \lambda_{j}\right)^{2}} \times\left\{\left\|\eta^{\prime}\right\|_{L^{2}}^{2}+\|\eta\|_{L^{2}}^{2}\right\},
$$


So, we can prove that

$$
\left\|\eta^{\prime}\right\|_{L^{2}}+\|\eta\|_{L^{2}} \leq C \epsilon^{\frac{1}{2}}
$$

the final result then follows easily.

Secondly, we consider the following problem

$$
\begin{gathered}
\epsilon^{2}\left[\beta^{-2} e^{\prime \prime}+\hbar_{5}(\theta) e^{\prime}\right]+\lambda_{0} e=\tilde{\mathbf{g}}, \quad \forall 0<\theta<1, \\
e^{\prime}(0)+\tilde{b}_{5} e(0)=0, \quad e^{\prime}(1)+\tilde{b}_{6} e(1)=0 .
\end{gathered}
$$

Lemma 7.2. If $\tilde{\mathrm{g}} \in L^{2}(0,1)$, then for all small $\epsilon$ satisfying (1.14) there is a unique solution $e \in H^{2}(0,1)$ to problem (7.19), which satisfies

$$
\|e\|_{b} \leq C \epsilon^{-1}\|\tilde{\mathbf{g}}\|_{L^{2}(0,1)} .
$$

Moreover, if $\tilde{\mathbf{g}} \in H^{2}(0,1)$, then

$$
\epsilon^{2}\left\|e^{\prime \prime}\right\|_{L^{2}(0,1)}+\epsilon\left\|e^{\prime}\right\|_{L^{2}(0,1)}+\|e\|_{L^{\infty}(0,1)} \leq C\|\tilde{\mathbf{g}}\|_{H^{2}(0,1)} .
$$

Proof. See Lemma 8.1 in [23].

Thirdly, we consider the following system

$$
\begin{array}{rr}
\mathbb{L}(f, e) \equiv\left(\mathbb{L}_{1}(f), \mathbb{L}_{2}(e)\right)=(\mathbf{g}(\theta), \tilde{\mathbf{g}}(\theta)), \quad 0<\theta<1, \\
f^{\prime}(1)+k_{2} f(1)=\Gamma_{1}^{1}, \quad f^{\prime}(0)+k_{1} f(0)=\Gamma_{0}^{1}, \\
e^{\prime}(1)+\tilde{b}_{6} e(1)=\Gamma_{1}^{0}, \quad e^{\prime}(0)+\tilde{b}_{5} e(0)=\Gamma_{0}^{0},
\end{array}
$$

where $\Gamma_{j}^{i}, i, j=0,1$ are some constants.

Lemma 7.3. Under the non-degenerate condition (2.7), if $\mathbf{g}, \tilde{\mathbf{g}} \in L^{2}(0,1)$, then there exists $\epsilon_{0}$ such that for all $0<\epsilon<\epsilon_{0}$ satisfying (1.14) there is a unique solution $(f, e)$ in $H^{2}(0,1)$ to problem (7.21) which satisfies

$$
\|f\|_{*}+\|e\|_{* *} \leq\left[\|\mathbf{g}\|_{L^{2}(0,1)}+\epsilon^{-1}\|\tilde{\mathbf{g}}\|_{L^{2}(0,1)}+\sum_{i, j=0}^{1}\left|\Gamma_{j}^{i}\right|\right] .
$$

Proof. See Lemma 7.3 in [61].

Proof of Theorem 1.1. Let us observe now that the linear operator

$$
\mathbb{L}^{*}(f, e)=\left(\mathbb{L}_{1}(f)-\hbar_{3}(\epsilon z) e-\epsilon^{2} \hbar_{4}(\epsilon z) e^{\prime \prime}, \mathbb{L}_{2}(e)\right),
$$

is invertible with bounds for $\mathbb{L}^{*}(f, e)=(\mathbf{g}, \tilde{\mathbf{g}})$ given by

$$
\|f\|_{*}+\|e\|_{* *} \leq C\|\mathbf{g}\|_{L^{2}(0,1)}+\epsilon^{-1}\|\tilde{\mathbf{g}}\|_{L^{2}(0,1)} .
$$

From (7.9), $\epsilon \mathbf{A}_{1, \epsilon}$ and $\epsilon^{2} \mathbf{A}_{2, \epsilon}$ are contraction mappings of their arguments in $\mathcal{F}$. By Banach Contraction Mapping Theorem and Lemma 7.3, we can solve the nonlinear problem

$$
\left[\mathbb{L}^{*}-\left(\epsilon \mathbf{A}_{1, \epsilon}, \epsilon^{2} \mathbf{A}_{2, \epsilon}\right)\right](f, e)=(\mathbf{g}, \tilde{\mathbf{g}})
$$


with the boundary conditions defined in (7.5)-(7.8) is uniquely solvable for $(f, e) \in \mathcal{F}$ provided that

$$
\|\mathbf{g}\|_{L^{2}(0,1)}<\epsilon^{1 / 2+\rho} \quad \text { and }\|\tilde{\mathbf{g}}\|_{L^{2}(0,1)}<\epsilon^{3 / 2+\rho},
$$

with some small positive constants $\rho$. The desired result for full problem (7.3)-(7.8) then follows directly from Schauder's fixed-point Theorem.

Acknowledgements: J. Yang is supported by NSFC(No.11371254). Part of this work was done when the authors visited Chern Institute of Mathematics, Nankai University in summer of 2014: we are very grateful to the institution for the kind hospitality.

\section{Appendices}

\section{A Proofs of Lemmas 2.1 and 2.2}

Proof of Lemma 2.1: In fact, the curves can be expressed in the following forms

$$
\begin{aligned}
\mathcal{C}_{1} & : \mathbb{H}\left(\tilde{t}, \tilde{\varphi}_{1}(\tilde{t})\right)=\gamma\left(\tilde{\varphi}_{1}(\tilde{t})\right)+\tilde{t} n\left(\tilde{\varphi}_{1}(\tilde{t})\right), \\
\mathcal{C}_{2} & : \mathbb{H}\left(\tilde{t}, \tilde{\varphi}_{2}(\tilde{t})\right)=\gamma\left(\tilde{\varphi}_{2}(\tilde{t})\right)+\tilde{t} n\left(\tilde{\varphi}_{2}(\tilde{t})\right), \\
\Gamma & : \mathbb{H}(0, \tilde{\theta})=\gamma(\tilde{\theta}) .
\end{aligned}
$$

It follows that the tangent vectors of $\mathcal{C}_{1}$ at $P_{1}$ can be written as

$$
\left.\frac{\mathrm{d} \mathcal{C}_{1}}{\mathrm{~d} \tilde{t}}\right|_{\tilde{t}=0}=\left.\left.\frac{\partial \gamma}{\partial \tilde{\theta}}\right|_{\tilde{\theta}=\tilde{\varphi}_{1}(0)} \cdot \frac{\mathrm{d} \tilde{\varphi}_{1}}{\mathrm{~d} \tilde{t}}\right|_{\tilde{t}=0}+n\left(\tilde{\varphi}_{1}(0)\right),
$$

and the tangent vector of $\Gamma$ at $P_{1}$ is

$$
\left.\frac{\partial \gamma}{\partial \tilde{\theta}}\right|_{\tilde{\theta}=\tilde{\varphi}_{1}(0)} .
$$

According to the condition: $\Gamma \perp \partial \Omega$, we have that

$$
\left\langle\left.\frac{\mathrm{d} \mathcal{C}_{1}}{\mathrm{~d} \tilde{t}}\right|_{\tilde{t}=0},\left.\frac{\partial \gamma}{\partial \tilde{\theta}}\right|_{\tilde{\theta}=\tilde{\varphi}_{1}(0)}\right\rangle=0 .
$$

Combining with

$$
\left\langle n\left(\tilde{\varphi}_{1}(0)\right),\left.\frac{\partial \gamma}{\partial \tilde{\theta}}\right|_{\tilde{\theta}=\tilde{\varphi}_{1}(0)}\right\rangle=0
$$

we have

$$
\tilde{\varphi}_{1}^{\prime}(0)=0
$$

Similarly, we can show $\tilde{\varphi}_{2}^{\prime}(0)=0$.

In the coordinate system $\left(y_{1}, y_{2}\right), \gamma(\tilde{\theta})$ and $n(\tilde{\theta})$ can be expressed as follows

$$
\gamma(\tilde{\theta})=\left(\gamma_{1}(\tilde{\theta}), \gamma_{2}(\tilde{\theta})\right), \quad n(\tilde{\theta})=\left(n_{1}(\tilde{\theta}), n_{2}(\tilde{\theta})\right) .
$$

The relations

$$
\left|\gamma_{1}^{\prime}(\tilde{\theta})\right|^{2}+\left|\gamma_{2}^{\prime}(\tilde{\theta})\right|^{2}=1, \quad\left|n_{1}(\tilde{\theta})\right|^{2}+\left|n_{2}(\tilde{\theta})\right|^{2}=1, \quad \gamma_{1}^{\prime}(\tilde{\theta}) n_{1}(\tilde{\theta})+\gamma_{2}^{\prime}(\tilde{\theta}) n_{2}(\tilde{\theta})=0,
$$


will give that

$$
\gamma_{1}^{\prime}(\tilde{\theta}) n_{2}(\tilde{\theta})-\gamma_{2}^{\prime}(\tilde{\theta}) n_{1}(\tilde{\theta})=1,
$$

provided that the sign is taken as ' + ' by suitable choice of the natural parameter of $\Gamma$. Then, the curve $\mathcal{C}_{1}$ can be expressed in the following form

$$
\begin{aligned}
\mathcal{C}_{1}: \mathbb{H}\left(\tilde{t}, \tilde{\varphi}_{1}(\tilde{t})\right) & =\left(\gamma_{1}\left(\tilde{\varphi}_{1}(\tilde{t})\right)+\tilde{t} n_{1}\left(\tilde{\varphi}_{1}(\tilde{t})\right), \gamma_{2}\left(\tilde{\varphi}_{1}(\tilde{t})\right)+\tilde{t} n_{2}\left(\tilde{\varphi}_{1}(\tilde{t})\right)\right) \\
& \equiv\left(y_{1}(\tilde{t}), y_{2}(\tilde{t})\right)
\end{aligned}
$$

The calculations

$$
\begin{gathered}
y_{1}^{\prime}(\tilde{t})=\gamma_{1}^{\prime}\left(\tilde{\varphi}_{1}\right) \cdot \frac{\mathrm{d} \tilde{\varphi}_{1}}{\mathrm{~d} \tilde{t}}+n_{1}\left(\tilde{\varphi}_{1}\right)+\tilde{t} \cdot n_{1}^{\prime}\left(\tilde{\varphi}_{1}\right) \cdot \frac{\mathrm{d} \tilde{\varphi}_{1}}{\mathrm{~d} \tilde{t}}, \\
y_{2}^{\prime}(\tilde{t})=\gamma_{2}^{\prime}\left(\tilde{\varphi}_{1}\right) \cdot \frac{\mathrm{d} \tilde{\varphi}_{1}}{\mathrm{~d} \tilde{t}}+n_{2}\left(\tilde{\varphi}_{1}\right)+\tilde{t} \cdot n_{2}^{\prime}\left(\tilde{\varphi}_{1}\right) \cdot \frac{\mathrm{d} \tilde{\varphi}_{1}}{\mathrm{~d} \tilde{t}}, \\
y_{1}^{\prime \prime}(\tilde{t})=\gamma_{1}^{\prime \prime}\left(\tilde{\varphi}_{1}\right) \cdot\left(\frac{\mathrm{d} \tilde{\varphi}_{1}}{\mathrm{~d} \tilde{t}}\right)^{2}+\gamma_{1}^{\prime}\left(\tilde{\varphi}_{1}\right) \cdot \frac{\mathrm{d}^{2} \tilde{\varphi}_{1}}{\mathrm{~d} \tilde{t}^{2}}+2 n_{1}^{\prime}\left(\tilde{\varphi}_{1}\right) \cdot \frac{\mathrm{d} \tilde{\varphi}_{1}}{\mathrm{~d} \tilde{t}} \\
+\tilde{t} n_{1}^{\prime \prime}\left(\tilde{\varphi}_{1}\right) \cdot\left(\frac{\mathrm{d} \tilde{\varphi}_{1}}{\mathrm{~d} \tilde{t}}\right)^{2}+\tilde{t} n_{1}^{\prime}\left(\tilde{\varphi}_{1}\right) \cdot \frac{\mathrm{d}^{2} \tilde{\varphi}_{1}}{\mathrm{~d} \tilde{t}^{2}}, \\
y_{2}^{\prime \prime}(\tilde{t})= \\
\gamma_{2}^{\prime \prime}\left(\tilde{\varphi}_{1}\right) \cdot\left(\frac{\mathrm{d} \tilde{\varphi}_{1}}{\mathrm{~d} \tilde{t}}\right)^{2}+\gamma_{2}^{\prime}\left(\tilde{\varphi}_{1}\right) \cdot \frac{\mathrm{d}^{2} \tilde{\varphi}_{1}}{\mathrm{~d} \tilde{t}^{2}}+2 n_{2}^{\prime}\left(\tilde{\varphi}_{1}\right) \cdot \frac{\mathrm{d} \tilde{\varphi}_{1}}{\mathrm{~d} \tilde{t}} \\
+\tilde{t} n_{2}^{\prime \prime}\left(\tilde{\varphi}_{1}\right) \cdot\left(\frac{\mathrm{d} \tilde{\varphi}_{1}}{\mathrm{~d} \tilde{t}}\right)^{2}+\tilde{t} n_{2}^{\prime}\left(\tilde{\varphi}_{1}\right) \cdot \frac{\mathrm{d}^{2} \tilde{\varphi}_{1}}{\mathrm{~d} \tilde{t}^{2}},
\end{gathered}
$$

imply that

$$
\left(y_{1}^{\prime}(t)\right)^{2}+\left.\left(y_{2}^{\prime}(t)\right)^{2}\right|_{\tilde{t}=0}=|n(0)|^{2}=1,
$$

and

$$
y_{1}^{\prime}(\tilde{t}) y_{2}^{\prime \prime}(\tilde{t})-\left.y_{1}^{\prime \prime}(\tilde{t}) y_{2}^{\prime}(\tilde{t})\right|_{\tilde{t}=0}=\left(n_{1}(0) \gamma_{2}^{\prime}(0)-n_{2}(0) \gamma_{1}^{\prime}(0)\right) \tilde{\varphi}_{1}^{\prime \prime}(0)=\tilde{\varphi}_{1}^{\prime \prime}(0) .
$$

Therefore, the signed curvature of the curve $\mathcal{C}_{1}$ at the point $P_{1}$ is

$$
k_{1}=\frac{y_{1}^{\prime}(0) y_{2}^{\prime \prime}(0)-y_{1}^{\prime \prime}(0) y_{2}^{\prime}(0)}{\left(\left(y_{1}^{\prime}(0)\right)^{2}+\left(y_{2}^{\prime}(0)\right)^{2}\right)^{\frac{3}{2}}}=\tilde{\varphi}_{1}^{\prime \prime}(0) \text {. }
$$

Similarly, we can show $k_{2}=\tilde{\varphi}_{2}^{\prime \prime}(0)$.

Proof of Lemma 2.2: Indeed, first comes the derivative of first order

$$
\begin{aligned}
\frac{\partial F}{\partial t}(0, \theta) & =\left.\left[\gamma^{\prime}(\Theta) \cdot \Theta_{t}+n(\Theta)+t n^{\prime}(\Theta) \cdot \Theta_{t}\right]\right|_{(0, \theta)} \\
& =\gamma^{\prime}(\Theta) \cdot 0+n(\Theta(0, \theta)) \\
& =n(\theta)
\end{aligned}
$$


So as $t$ is small enough, $\frac{\partial F}{\partial t} \neq 0$. Consider the derivative of second order

$$
\begin{aligned}
q_{1}(\theta) & \equiv \frac{\partial^{2} F}{\partial t^{2}}(0, \theta) \\
& =\left.\left[\gamma^{\prime \prime}(\Theta) \cdot\left(\Theta_{t}\right)^{2}+\gamma^{\prime}(\Theta) \cdot \Theta_{t t}+2 n^{\prime}(\Theta) \cdot \Theta_{t}+t n^{\prime \prime}(\Theta) \cdot\left(\Theta_{t}\right)^{2}+t n^{\prime}(\Theta) \cdot \Theta_{t t}\right]\right|_{(0, \theta)} \\
& =\gamma^{\prime}(\theta) \cdot \Theta_{t t}(0, \theta) \perp n(\gamma(\theta)) .
\end{aligned}
$$

Moreover, there hold

$$
\begin{aligned}
q_{1}^{\prime}(\theta) & =\left.\left[\gamma^{\prime \prime}(\Theta) \cdot \Theta_{\theta} \cdot \Theta_{t t}+\left.\gamma^{\prime}(\Theta) \cdot \Theta_{t t \theta}\right|_{(0, \theta)}\right]\right|_{(0, \theta)} \\
& =\left.\gamma^{\prime \prime}(\Theta) \cdot \Theta_{t t}\right|_{(0, \theta)}+\left.\gamma^{\prime}(\Theta) \Theta_{t t \theta}\right|_{(0, \theta)},
\end{aligned}
$$

and specially,

$$
q_{1}^{\prime}(0)=\gamma^{\prime \prime}(0) k_{1}+\gamma^{\prime}(0)\left(k_{2}-k_{1}\right), \quad q_{1}^{\prime}(1)=\gamma^{\prime \prime}(1) k_{2}+\gamma^{\prime}(1)\left(k_{2}-k_{1}\right) .
$$

The derivative of third order is

$$
\begin{aligned}
q_{2}(\theta) \equiv & \frac{\partial^{3} F}{\partial t^{3}}(0, \theta) \\
= & {\left[\gamma^{\prime \prime \prime}(\Theta) \cdot\left(\Theta_{t}\right)^{3}+3 \gamma^{\prime \prime}(\Theta) \cdot \Theta_{t} \cdot \Theta_{t t}+\gamma^{\prime}(\Theta) \cdot \Theta_{t t t}+3 n^{\prime \prime}(\Theta) \cdot\left(\Theta_{t}\right)^{2}\right.} \\
& \left.\quad+3 n^{\prime}(\Theta) \cdot \Theta_{t t}\right]\left.\right|_{(0, \theta)} \\
= & \gamma^{\prime}(\theta) \cdot \Theta_{t t t}(0, \theta)+3 n^{\prime}(\theta) \cdot \Theta_{t t}(0, \theta) \quad \perp n(\theta) .
\end{aligned}
$$

This finishes the proof of the lemma.

As a conclusion, as $t$ is small enough, there hold the asymptotic behavior

$$
F(t, \theta)=\gamma(\theta)+\operatorname{tn}(\theta)+\frac{t^{2}}{2} q_{1}(\theta)+\frac{t^{3}}{6} q_{2}(\theta)+O\left(t^{4}\right), \quad \forall \theta \in[0,1], t \in\left(-\delta_{0}, \delta_{0}\right),
$$

where $\delta_{0}>0$ is a small constant. This gives us that

$$
\begin{gathered}
\frac{\partial F}{\partial t}(t, \theta)=n(\theta)+t q_{1}(\theta)+\frac{t^{2}}{2} q_{2}(\theta)+O\left(t^{3}\right), \\
\frac{\partial F}{\partial \theta}(t, \theta)=\gamma^{\prime}(\theta)-k(\theta) t \gamma^{\prime}(\theta)+\frac{t^{2}}{2} q_{1}^{\prime}(\theta)+\frac{t^{3}}{6} q_{2}^{\prime}(\theta)+O\left(t^{4}\right) .
\end{gathered}
$$

Specially, there hold$$
\frac{\partial F}{\partial t}(t, 0)=n(0)+t q_{1}(0)+\frac{t^{2}}{2} q_{2}(0)+O\left(t^{3}\right),
$$$$
\frac{\partial F}{\partial t}(t, 1)=n(1)+t q_{1}(1)+\frac{t^{2}}{2} q_{2}(1)+O\left(t^{3}\right)
$$$$
\frac{\partial F}{\partial \theta}(t, 0)=\gamma^{\prime}(0)-k(0) t \gamma^{\prime}(0)+\frac{t^{2}}{2} q_{1}^{\prime}(0)+\frac{t^{3}}{6} q_{2}^{\prime}(0)+O\left(t^{4}\right),
$$$$
\frac{\partial F}{\partial \theta}(t, 1)=\gamma^{\prime}(1)-k(1) t \gamma^{\prime}(1)+\frac{t^{2}}{2} q_{1}^{\prime}(1)+\frac{t^{3}}{6} q_{2}^{\prime}(1)+O\left(t^{4}\right) .
$$

These formulas will play an important role in the derivation of the local form of (1.1), which will be given in Appendix B. 


\section{B Local forms of the differential operators in (1.1)}

In this section, we are devoted to presenting the expressions of the differential operators $\Delta$ and $\partial / \partial \nu$ in (1.1). The formulas will be provided in the local forms in the modified Fermi coordinates given in Section 2.

We first derive the metric matrix. Note that

$$
\gamma^{\prime}(\theta) \perp n(\theta), \quad n^{\prime}(\theta) \perp n(\theta), \quad q_{1}(\theta) \perp n(\theta), \quad q_{2}(\theta) \perp n(\theta) .
$$

As the first step, here are the computation of the metric matrix:

$$
\begin{aligned}
g_{11} & =\left\langle\frac{\partial F}{\partial t}, \frac{\partial F}{\partial t}\right\rangle \\
& =\left\langle n+t q_{1}+\frac{t^{2}}{2} q_{2}+O\left(t^{3}\right), n+t q_{1}+\frac{t^{2}}{2} q_{2}+O\left(t^{3}\right)\right\rangle \\
& =1+t^{2}\left|q_{1}\right|^{2}+O\left(t^{3}\right),
\end{aligned}
$$

and

$$
\begin{aligned}
g_{12} & =\left\langle\frac{\partial F}{\partial t}, \frac{\partial F}{\partial \theta}\right\rangle \\
& =\left\langle n+t q_{1}+\frac{t^{2}}{2} q_{2}+O\left(t^{3}\right), \gamma^{\prime}-k t \gamma^{\prime}+\frac{t^{2}}{2} q_{1}^{\prime}+O\left(t^{3}\right)\right\rangle \\
& =\frac{t^{2}}{2}<n, q_{1}^{\prime}>+t<q_{1}, \gamma^{\prime}>-t^{2} k<q_{1}, \gamma^{\prime}>+\frac{t^{2}}{2}<q_{2}, \gamma^{\prime}>+O\left(t^{3}\right) \\
& =t<q_{1}, \gamma^{\prime}>+\frac{t^{2}}{2}\left(<q_{2}, \gamma^{\prime}>-k<q_{1}, \gamma^{\prime}>\right)+O\left(t^{3}\right),
\end{aligned}
$$

where we have used the fact

$$
<q_{1}^{\prime}, n>-k<q_{1}, \gamma^{\prime}>=<q_{1}^{\prime}, n>+<q_{1}, n^{\prime}>=\frac{\partial}{\partial \theta}<q_{1}, n>=0 .
$$

The last element is

$$
\begin{aligned}
g_{22} & =\left\langle\frac{\partial F}{\partial \theta}, \frac{\partial F}{\partial \theta}\right\rangle \\
& =\left\langle\gamma^{\prime}-k t \gamma^{\prime}+\frac{t^{2}}{2} q_{1}^{\prime}+O\left(t^{3}\right), \gamma^{\prime}-k t \gamma^{\prime}+\frac{t^{2}}{2} q_{1}^{\prime}+O\left(t^{3}\right)\right\rangle \\
& \left.=1-2 k t+t^{2}\left(<q_{1}^{\prime}, \gamma^{\prime}\right\rangle+k^{2}\right)+O\left(t^{3}\right) .
\end{aligned}
$$

So the determinant of the metric matrix is

$$
\begin{aligned}
g & =\operatorname{det}\left(g_{i j}\right) \\
& =1-2 k t+t^{2}\left(<q_{1}^{\prime}, \gamma^{\prime}>+k^{2}\right)+t^{2}\left|q_{1}\right|^{2}-t^{2}<q_{1}, \gamma^{\prime}>^{2}+O\left(t^{3}\right) \\
& =1-2 k t+t^{2}\left(<q_{1}^{\prime}, \gamma^{\prime}>+k^{2}\right)+O\left(t^{3}\right),
\end{aligned}
$$

where we have used $\left.\left|q_{1}\right|^{2}-<q_{1}, \gamma^{\prime}\right\rangle^{2}=0$ due to the expression of $q_{1}$ in Lemma 2.2. 
We turn to the computation of the inverse of the metric matrix. By

$$
\left[1+a t+b t^{2}+O\left(t^{3}\right)\right]^{-1}=1-a t+\left(a^{2}-b\right) t^{2}+O\left(t^{3}\right),
$$

we have

$$
g^{-1}=1+2 k t+t^{2}\left(3 k^{2}-<q_{1}^{\prime}, \gamma^{\prime}>\right)+O\left(t^{3}\right) .
$$

Whence

$$
\begin{aligned}
& \begin{aligned}
g^{11} & =g_{22} \cdot g^{-1} \\
& =1-2 k t+t^{2}\left(<q_{1}^{\prime}, \gamma^{\prime}>+k^{2}\right)+2 k t-4 k^{2} t^{2}+t^{2}\left(3 k^{2}-<q_{1}^{\prime}, \gamma^{\prime}>\right)+O\left(t^{3}\right) \\
& =1+O\left(t^{3}\right), \\
-g^{12}= & g_{12} \cdot g^{-1} \\
= & t<q_{1}, \gamma^{\prime}>+2 k t^{2}<q_{1}, \gamma^{\prime}>+\frac{t^{2}}{2}\left(<q_{2}^{\prime}, \gamma^{\prime}>-k<q_{1}, \gamma^{\prime}>\right)+O\left(t^{3}\right) \\
= & t<q_{1}, \gamma^{\prime}>+\frac{t^{2}}{2}\left(3 k<q_{1}, \gamma^{\prime}>+<q_{2}, \gamma^{\prime}>\right)+O\left(t^{3}\right), \\
g^{22}= & g_{11} \cdot g^{-1}=1+2 k t+t^{2}\left(3 k^{2}-<q_{1}^{\prime}, \gamma^{\prime}>+\left|q_{1}\right|^{2}\right)+O\left(t^{3}\right) .
\end{aligned}
\end{aligned}
$$

We can get the following formulas

$$
\begin{aligned}
\sqrt{g} & =1-k t+\frac{1}{2} t^{2}<q_{1}^{\prime}, \gamma^{\prime}>+O\left(t^{3}\right) \\
(\sqrt{g})^{-1} & =1+k t+\frac{1}{2} t^{2}\left(2 k^{2}-<q_{1}^{\prime}, \gamma^{\prime}>\right)+O\left(t^{3}\right), \\
\sqrt{g^{22}} & =1+k t+\frac{1}{2} t^{2}\left(3 k^{2}-<q_{1}^{\prime}, \gamma^{\prime}>+\left|q_{1}\right|^{2}-\frac{1}{4} \cdot 4 k^{2}\right)+O\left(t^{3}\right) \\
& =1+k t+\frac{1}{2}\left(2 k^{2}-<q_{1}^{\prime}, \gamma^{\prime}>+\left|q_{1}\right|^{2}\right) t^{2}+O\left(t^{3}\right), \\
\frac{1}{\sqrt{g^{22}}} & =1-k t+\left(k^{2}-k^{2}+\frac{1}{2}<q_{1}^{\prime}, \gamma^{\prime}>-\frac{1}{2}\left|q_{1}\right|^{2}\right) t^{2}+O\left(t^{3}\right) \\
& =1-k t+\frac{1}{2}\left(<q_{1}^{\prime}, \gamma^{\prime}>-\left|q_{1}\right|^{2}\right) t^{2}+O\left(t^{3}\right) .
\end{aligned}
$$

Now, recall the definition of Laplace-Beltrami operator in the form

$$
\begin{aligned}
\triangle_{t, \theta} u= & g^{11} \frac{\partial^{2} u}{\partial t^{2}}+2 g^{12} \frac{\partial^{2} u}{\partial t \partial \theta}+g^{22} \frac{\partial^{2} u}{\partial \theta^{2}}+(\sqrt{g})^{-1}\left[\frac{\partial}{\partial t}\left(\sqrt{g} g^{11}\right)+\frac{\partial}{\partial \theta}\left(\sqrt{g} g^{21}\right)\right] \frac{\partial u}{\partial t} \\
& +(\sqrt{g})^{-1}\left[\frac{\partial}{\partial t}\left(\sqrt{g} g^{12}\right)+\frac{\partial}{\partial \theta}\left(\sqrt{g} g^{22}\right)\right] \frac{\partial u}{\partial \theta} .
\end{aligned}
$$


The last two coefficients in (B.1) can be computed as follows. Since

$$
\sqrt{g} \cdot g^{11}=1-k t+\frac{t^{2}}{2}<q_{1}^{\prime}, \gamma^{\prime}>+O\left(t^{3}\right)
$$

we can get

$$
\frac{\partial}{\partial t}\left(\sqrt{g} g^{11}\right)=-k+t<q_{1}^{\prime}, \gamma^{\prime}>+O\left(t^{2}\right)
$$

Since

$$
\begin{aligned}
\sqrt{g} \cdot g^{12} & =-t<q_{1}, \gamma^{\prime}>-\frac{t^{2}}{2}\left(3 k<q_{1}, \gamma^{\prime}>+<\gamma^{\prime}, q_{2}>\right)+k t^{2}<q_{1}, \gamma^{\prime}>+O\left(t^{3}\right) \\
& =-t<q_{1}, \gamma^{\prime}>-\frac{t^{2}}{2}\left(k<q_{1}, \gamma^{\prime}>+<\gamma^{\prime}, q_{2}>\right)+O\left(t^{3}\right)
\end{aligned}
$$

we can get

$$
\begin{aligned}
& \frac{\partial}{\partial \theta}\left(\sqrt{g} \cdot g^{12}\right)=-t<q_{1}, \gamma^{\prime}>^{\prime}-\frac{t^{2}}{2}\left(k<q_{1}, \gamma^{\prime}>+<\gamma^{\prime}, q_{2}>\right)^{\prime}+O\left(t^{3}\right), \\
& \frac{\partial}{\partial t}\left(\sqrt{g} \cdot g^{12}\right)=-<q_{1}, \gamma^{\prime}>-t\left(k<q_{1}, \gamma^{\prime}>+<\gamma^{\prime}, q_{2}>\right)+O\left(t^{2}\right) .
\end{aligned}
$$

So

$$
\begin{aligned}
\frac{\partial}{\partial t}\left(\sqrt{g} \cdot g^{11}\right)+\frac{\partial}{\partial \theta}\left(\sqrt{g} \cdot g^{12}\right) & =-k+t\left(<q_{1}^{\prime}, \gamma^{\prime}>-<q_{1}, \gamma^{\prime}>^{\prime}\right)+O\left(t^{2}\right) \\
& =-k+O\left(t^{2}\right)
\end{aligned}
$$

where we have used the relations

$$
<q_{1}^{\prime}, \gamma^{\prime}>-<q_{1}, \gamma^{\prime}>^{\prime}=-<q_{1}, \gamma^{\prime \prime}>=0
$$

We obtain the coefficient of the first bracket in (B.1) is

$$
(\sqrt{g})^{-1}\left(\frac{\partial}{\partial t}\left(\sqrt{g} \cdot g^{11}\right)+\frac{\partial}{\partial \theta}\left(\sqrt{g} \cdot g^{12}\right)\right)=-k-k^{2} t+O\left(t^{2}\right) .
$$

On the other hand, by

$$
\begin{aligned}
\sqrt{g} \cdot g^{22} & =1-k t+\frac{t^{2}}{2}<q_{1}, \gamma^{\prime}>+2 k t-2 k^{2} t^{2}+t^{2}\left(3 k^{2}-<q_{1}, \gamma^{\prime}>+\left|q_{1}\right|^{2}\right)+O\left(t^{3}\right) \\
& =1+k t+\frac{t^{2}}{2}\left(2 k^{2}-<q_{1}, \gamma^{\prime}>+2\left|q_{1}\right|^{2}\right)+O\left(t^{3}\right),
\end{aligned}
$$

we can get

$$
\frac{\partial}{\partial \theta}\left(\sqrt{g} \cdot g^{22}\right)=k^{\prime} t+\frac{t^{2}}{2}\left(2 k^{2}-<q_{1}, \gamma^{\prime}>+2\left|q_{1}\right|^{2}\right)^{\prime}+O\left(t^{3}\right) .
$$

This implies that

$$
\frac{\partial}{\partial t}\left(\sqrt{g} \cdot g^{12}\right)+\frac{\partial}{\partial \theta}\left(\sqrt{g} \cdot g^{22}\right)=-<q_{1}, \gamma^{\prime}>+t\left(k^{\prime}-k<q_{1}, \gamma^{\prime}>-<q_{2}, \gamma^{\prime}>\right)+O\left(t^{2}\right),
$$


which gives the coefficient of the second bracket in (B.1) is

$$
\begin{aligned}
& (\sqrt{g})^{-1}\left(\frac{\partial}{\partial t}\left(\sqrt{g} \cdot g^{12}\right)+\frac{\partial}{\partial \theta}\left(\sqrt{g} \cdot g^{22}\right)\right) \\
& =-<q_{1}, \gamma^{\prime}>+t\left(k^{\prime}-2 k<q_{1}, \gamma^{\prime}>-<q_{2}, \gamma^{\prime}>\right)+O\left(t^{2}\right) .
\end{aligned}
$$

Denote

$$
\varpi(\theta) \equiv<q_{1}(\theta), \gamma^{\prime}(\theta)>=\Theta_{t t}(0, \theta)
$$

where we have used the expression of $q_{1}$ in Lemma 2.2. Hence, the term $\triangle_{y} u$ in (1.1) has the following form in the modified Fermi coordinate system

$$
\begin{aligned}
\triangle_{y} u= & {\left[1+O\left(t^{3}\right)\right] u_{t t}-2\left[\varpi t+\frac{t^{2}}{2}\left(3 k \varpi+<q_{2}, \gamma^{\prime}>\right)+O\left(t^{3}\right)\right] u_{t \theta} } \\
& +\left[1+2 k t+t^{2}\left(3 k^{2}-<q_{1}^{\prime}, \gamma^{\prime}>+\left|q_{1}\right|^{2}\right)+O\left(t^{3}\right)\right] u_{\theta \theta} \\
& +\left[-k-k^{2} t+O\left(t^{2}\right)\right] u_{t}+\left[-\varpi+t k^{\prime}-t\left(2 k a_{0}+<q_{2}, \gamma^{\prime}>\right)+O\left(t^{2}\right)\right] u_{\theta} \\
\equiv & \left(1+a_{2} t^{3}\right) u_{t t}+\left(-2 \varpi t+a_{3} t^{2}\right) u_{t \theta}+\left(1+2 k t+a_{1} t^{2}\right) u_{\theta \theta} \\
& +\left(-k-k^{2} t+a_{4} t^{2}\right) u_{t}+\left(-\varpi+a_{5} t\right) u_{\theta} .
\end{aligned}
$$

The terms in $\triangle_{y} u$ will be rearranged in the form

$$
\triangle_{y} u=u_{t t}+u_{\theta \theta}+\bar{B}_{1}(u)+\bar{B}_{0}(u),
$$

where

$$
\bar{B}_{1}(u)=-\left(k+k^{2} t\right) u_{t}-2 \varpi t u_{t \theta}-\varpi u_{\theta},
$$

and

$$
\bar{B}_{0}(u)=2 k t u_{\theta \theta}+a_{1} t^{2} u_{\theta \theta}+a_{2} t^{3} u_{t t}+a_{3} t^{2} u_{t \theta}+a_{4} t^{2} u_{t}+a_{5} t u_{\theta} .
$$

We finally show the expression of $\nu$, the outward unit normal of $\partial \Omega$ near $P_{1}, P_{2}$, i.e., when $\theta=0,1$ in the modified Fermi coordinates. This will provide the local expression of $\partial u / \partial \nu$ in (1.1). Suppose

$$
\nu=\sigma_{1} \frac{\partial F}{\partial t}+\sigma_{2} \frac{\partial F}{\partial \theta} .
$$

Since $\partial F / \partial t \in T(\partial \Omega)$, we have $<\partial F / \partial t, \nu>=0$. Hence

$$
\sigma_{2} \neq 0, \quad \frac{\partial F}{\partial \theta} \neq 0
$$

and

$$
\sigma_{1} g_{11}+\sigma_{2} g_{12}=0
$$

On the other hand, $\langle\nu, \nu\rangle=1$, that is

$$
\left\langle\sigma_{1} \frac{\partial F}{\partial t}+\sigma_{2} \frac{\partial F}{\partial \theta}, \sigma_{1} \frac{\partial F}{\partial t}+\sigma_{2} \frac{\partial F}{\partial \theta}\right\rangle=1,
$$


which implies that

$$
\sigma_{1}^{2} g_{11}+\sigma_{2}^{2} g_{22}+2 \sigma_{1} \sigma_{2} g_{12}=1
$$

Combining above two equations, one can get

$$
\sigma_{1}= \pm \frac{g^{12}}{\sqrt{g^{22}}}, \quad \sigma_{2}= \pm \sqrt{g^{22}}
$$

Recall that

$$
\begin{gathered}
q_{1}(\theta)=\gamma^{\prime}(\theta)\left[\left(k_{2}-k_{1}\right) \theta+k_{1}\right] \\
q_{1}^{\prime}(\theta)=\gamma^{\prime \prime}(\theta)\left[\left(k_{2}-k_{1}\right) \theta+k_{1}\right]+\gamma^{\prime}(\theta)\left(k_{2}-k_{1}\right) ; \\
q_{2}(\theta)=\gamma^{\prime}(\theta)\left[\left(\tilde{\varphi}_{2}^{\prime \prime \prime}(0)-\tilde{\varphi}_{1}^{\prime \prime \prime}(0)\right) \theta+\tilde{\varphi}_{1}^{\prime \prime \prime}(0)\right]-3 k \gamma^{\prime}(\theta)\left[\left(k_{2}-k_{1}\right) \theta+k_{1}\right] .
\end{gathered}
$$

By choosing the + , it is easy to check that

$$
\begin{aligned}
\sigma_{2} & =1+k t+\frac{1}{2}\left(2 k^{2}-<q_{1}^{\prime}, \gamma^{\prime}>+\left|q_{1}\right|^{2}\right) t^{2}+O\left(t^{3}\right), \\
& =1+k t+k^{2} t^{2}-\frac{1}{2}\left(k_{2}-k_{1}\right) t^{2}+\frac{1}{2}\left[\left(k_{2}-k_{1}\right) \theta+k_{1}\right]^{2} t^{2}+O\left(t^{3}\right) .
\end{aligned}
$$

Similarly, we get

$$
\begin{aligned}
\sigma_{1}=- & \sigma_{2} \cdot g_{12} \cdot\left(g_{11}\right)^{-1} \\
=- & \left\{1+k t+k^{2} t^{2}-\frac{1}{2}\left(k_{2}-k_{1}\right) t^{2}+\frac{1}{2}\left[\left(k_{2}-k_{1}\right) \theta+k_{1}\right]^{2} t^{2}\right\} \\
& \times\left[\varpi t+\frac{1}{2}\left(<q_{2}, \gamma^{\prime}>-k a_{0}\right)\right]+O\left(t^{3}\right) \\
=- & {\left[\left(k_{2}-k_{1}\right) \theta+k_{1}\right] t+k\left[\left(k_{2}-k_{1}\right) \theta+k_{1}\right] t^{2}-\frac{1}{2}\left[\left(\tilde{\varphi}_{2}^{\prime \prime \prime}(0)-\tilde{\varphi}_{1}^{\prime \prime \prime}(0)\right) \theta+\tilde{\varphi}_{1}^{\prime \prime \prime}(0)\right] t^{2}+O\left(t^{3}\right) . }
\end{aligned}
$$

In the modified Fermi coordinates $(t, \theta)$ in (2.4), the normal derivative $\partial u / \partial \nu$ has a local form as follows

$$
\sigma_{1} \frac{\partial u}{\partial t}+\sigma_{2} \frac{\partial u}{\partial \theta}
$$

More precisely, for $\theta=0$, it is

$$
k_{1} t u_{t}+b_{1} t^{2} u_{t}-u_{\theta}-k(0) t u_{\theta}+b_{2} t^{2} u_{\theta}+\bar{D}_{0}^{0}(u),
$$

where

$$
\bar{D}_{0}^{0}(u)=\sigma_{3}(t) u_{t}+\sigma_{4}(t) u_{\theta},
$$

and the constants $b_{1}$ and $b_{2}$ are given by

$$
b_{1}=\frac{1}{2} \tilde{\varphi}_{1}^{\prime \prime \prime}(0)-k(0) k_{1}, \quad b_{2}=\frac{1}{2}\left(k_{2}-k_{1}\right)-k^{2}(0)-\frac{1}{2} k_{1}^{2} .
$$

On the other hand, for $\theta=1$, it has the form

$$
k_{2} t u_{t}+b_{3} t^{2} u_{t}-u_{\theta}-k(1) t u_{\theta}+b_{4} t^{2} u_{\theta}+\bar{D}_{0}^{1}(u),
$$


with the notation

$$
\begin{gathered}
\bar{D}_{0}^{1}(u)=\sigma_{5}(t) u_{t}+\sigma_{6}(t) u_{\theta}, \\
b_{3}=\frac{1}{2} \tilde{\varphi}_{2}^{\prime \prime \prime}(0)-k(1) k_{2}, \quad b_{4}=\frac{1}{2}\left(k_{2}-k_{1}\right)-k^{2}(1)-\frac{1}{2} k_{2}^{2} .
\end{gathered}
$$

In the above, the functions $\sigma_{3}, \cdots, \sigma_{6}$ are smooth functions of $t$ with the properties

$$
\left|\sigma_{i}(t)\right| \leq C|t|^{3}, \quad i=3,4,5,6 .
$$

\section{References}

[1] N. Alikakos, X. Chen and G. Fusco, Motion of a droplet by surface tension along the boundary, Calc. Var. Partial Differential Equations 11 (2000), no. 3, 233-305.

[2] A. Ambrosetti, M. Badiale and S. Cingolani, Semiclassical states of nonlinear Schrödinger equations, Arch. Rational Mech. Anal. 140 (1997), 285-300.

[3] A. Ambrosetti, A. Malchiodi and W.-M. Ni, Singularly perturbed elliptic equations with symmetry: existence of solutions concentrating on spheres I, Comm. Math. Phys. 235 (2003), no. $3,427-466$.

[4] A. Ambrosetti, A. Malchiodi and W.-M. Ni, Singularly perturbed elliptic equations with symmetry: existence of solutions concentrating on spheres II, Indiana Univ. Math. J. 53 (2004), no. 2, 297-329.

[5] A. Ambrosetti, A. Malchiodi and S. Secchi, Multiplicity results for some nonlinear Schrödinger equations with potentials, Arch. Rat. Mech. Anal. 159 (2001), 253-271.

[6] W. Ao, M. Musso and J. Wei, On spikes concentrating on line-segments to a semilinear Neumann problem, J. Differential Equations 251 (2011), no. 4-5, 881C901.

[7] W. Ao, M. Musso and J. Wei, Triple junction solutions for a singularly perturbed Neumann problem, SIAM J. Math. Anal. 43 (2011), no. 6, 2519-2541.

[8] W. Ao and J. Yang, Layered solutions with concentration on lines in three-dimensional domains, Anal. Appl. (Singap.) 12 (2014), no. 2, 161-194.

[9] T. Bartsch and S. Peng, Solutions concentrating on higher dimensional subsets for singularly perturbed elliptic equations II, J. Differential Equations 248 (2010), no. 11, 2746-2767.

[10] T. Bartsch and S. Peng, Solutions concentrating on higher dimensional subsets for singularly perturbed elliptic equations. I, Indiana Univ. Math. J. 57 (2008), no. 4, 1599-1631.

[11] P. Bates and G. Fusco, Equilibria with many nuclei for the Cahn-Hilliard equation, J. Differential Equations 160 (2000), no. 2, 283-356.

[12] P. Bates, E. N. Dancer and J. Shi, Multi-spike stationary solutions of the Cahn-Hilliard equation in higher-dimension and instability, Adv. Differential Equations 4 (1999), no. 1, $1-69$. 
[13] S. Cingolani and M. Lazzo, Multiple positive solutions to nonlinear Schrödinger equations with competing potential functions, J. Differential Equations, 160 (2000), no. 1, 118-138.

[14] E. N. Dancer and S. Yan, Multipeak solutions for a singularly perturbed Neumann problem, Pacific J. Math. 189 (1999), no. 2, 241-262.

[15] E. N. Dancer and S. Yan, Interior and boundary peak solutions for a mixed boundary value problem, Indiana Univ. Math. J. 48 (1999), no. 4, 1177-1212.

[16] E. N. Dancer and S. Yan, A new type of concentration solutions for a singularly perturbed elliptic problem, Trans. Amer. Math. Soc, 359 (2007), no. 4, 1765-1790.

[17] M. del Pino and P. Felmer, Local mountain passes for semilinear elliptic problems in unbounded domains, Calc. Var. Partial Differential Equations, 4 (1996), no. 2, 121-137.

[18] M. del Pino and P. Felmer, Semi-classical states for nonlinear Schrödinger equations, J. Funct. Anal. 149 (1997), no. 1, 245-265.

[19] M. del Pino and P. Felmer, Multi-peak bound states for nonlinear Schrödinger equations, Ann. Inst. H. Poincaré Anal. Non Linéaire 15 (1998), no. 2, 127-149.

[20] M. del Pino and P. Felmer, Semi-classical states of nonlinear Schrödinger equations: a variational reduction method, Math. Ann. 324 (2002), no. 1, 1-32.

[21] M. del Pino, P. Felmer and J. Wei, On the role of mean curvature in some singularly perturbed Neumann problems, SIAM J. Math. Anal. 31 (1999), no. 1, 63-79.

[22] M. del Pino, P. Felmer and J. Wei, On the role of distance function in some singular perturbation problems, Comm. Partial Differential Equations 25 (2000), no. 1-2, 155-177.

[23] M. del Pino, M. Kowalczyk and J. Wei, Concentration on curves for nonlinear Schrödinger equations, Comm. Pure Appl. Math. 60 (2007), no. 1, 113-146.

[24] T. D'Aprile, Solutions with many mixed positive and negative interior spikes for a semilinear Neumann problem, Calc. Var. Partial Differential Equations 41 (2011), no. 3-4, 435-454.

[25] T. D'Aprile and A. Pistoia, On the existence of some new positive interior spike solutions to a semilinear Neumann problem, J. Differential Equations 248 (2010), no. 3, 556-573.

[26] T. D'Aprile and A. Pistoia, Nodal clustered solutions for some singularly perturbed Neumann problems, Comm. Partial Differential Equations 35 (2010), no. 8, 1355-1401.

[27] M. P. do Carmo, Differential geometry of curves and surfaces, Translated from the Portuguese. Prentice-Hall, Inc., Englewood Cliffs, N. J., 1976.

[28] P. Felmer and J. J. Torres, Semi classical limits for the one dimensional nonlinear Schrödinger equation, Commun. Contemp. Math. 4 (2002), no. 3, 481-512.

[29] A. Floer and A. Weinstein, Nonspreading wave packets for the cubic Schrödinger equation with a bounded potential, J. Funct. Anal. 69 (1986), no. 3, 397-408.

[30] A. Gierer and H. Meinhardt, A thoery of biological pattern formation, Kybernetik(Berlin), 12 (1972), 30-39. 
[31] M. Grossi, A. Pistoia and J. Wei, Existence of multipeak solutions for a semilinear Neumann problem via nonsmooth critical point theory, Calc. Var. Partial Differential Equations 11 (2000), no. 2, 143-175.

[32] C. Gui and J. Wei, Multiple interior peak solutions for some singularly perturbed Neumann problems, J. Differential Equations 158 (1999), no. 1, 1-27.

[33] C. Gui and J. Wei, On multiple mixed interior and boundary peak solutions for some singularly perturbed Neumann problems, Canad. J. Math. 52 (2000), no. 3, 522-538.

[34] C. Gui, J.Wei and M. Winter, Multiple boundary peak solutions for some singularly perturbed Neumann problems, Ann. Inst. H. Poincaré Anal. Non Linéaire 17 (2000), no. 1, 47-82.

[35] Y. Guo and J. Yang, Concentration on surfaces for a singularly perturbed Neumann problem in three-dimensional domains, J. Differential Equations 255 (2013), no. 8, 2220-2266.

[36] R. Kohn and P. Sternberg, Local minimisers and singular perturbations, Proc. Roy. Soc. Edinburgh Sect. A 111 (1989), no. 1-2, 69-84.

[37] M. Kowalcayk, On the existence and Morse index of solutions to the Allen-Cahn equation in two dimensions, Ann. Mat. Pura Appl. (4) 184 (2005), no. 1, 17-52.

[38] M. Kowalczyk, Approximate invariant manifold of the Allen-Cahn flow in two dimensions. Partial differential equations and inverse problems, 233-239, Contemp. Math., 362, Amer. Math. Soc., Providence, RI, 2004.

[39] B. M. Levitan and I. S. Sargsjan, Sturm-Liouville and Dirac operator. Mathematics and its Applications (Soviet Series), 59. Kluwer Academic Publishers Group, Dordrecht, 1991.

[40] G. Li, S. Peng and S. Yan, A new type of solutions for a singularly perturbed elliptic Neumann Problem, Rev. Mat. Iberoam. 23 (2007), no. 3, 1039-1066.

[41] Y.-Y. Li, On a singularly perturbed equation with Neumann boundary condition, Comm. Partial Differential Equations 23 (1998), no. 3-4, 487-545.

[42] C.-S. Lin, W.-M. Ni and I. Takagi, Large amplitude stationary solutions to a chemotaxis system, J. Differential Equations 72 (1988), no. 1, 1-27.

[43] F. Mahmoudi and A. Malchiodi, Concentration on minimal submanifolds for a singularly perturbed Neumann problem, Adv. Math. 209 (2007), no. 2, 460-525.

[44] F. Mahmoudi, A. Malchiodi and M. Montenegro, Solutions to the nonlinear Schrödinger equation carrying momentum along a curve, Comm. Pure Appl. Math. 62 (2009), no.9, $1155-1264$.

[45] F. Mahmoudi, F. S. Sánchez and W. Yao, On the Ambrosetti-Malchiodi-Ni conjecture for general submanifolds, J. Differential Equations 258 (2015), no. 2, 243-280.

[46] A. Malchiodi, Solutions concentrating at curves for some singularly perturbed elliptic problems, C. R. Math. Acad. Sci. Parirs 338 (2004), no. 10, 775-780.

[47] A. Malchiodi, Concentration at curves for a singularly perturbed Neumann problem in threedimensiional domains, Geom. Funct. Anal. 15 (2005), no. 6, 1162-1222. 
[48] A. Malchiodi and M. Montenegro, Boundary concentration phenomena for a singularly perturbed elliptic problem, Comm. Pure Appl. Math, 55 (2002), no. 12, 1507-1568.

[49] A. Malchiodi and M. Montenegro, Multidimensional boundary layers for a singularly perturbed Neumann problem, Duke Math. J. 124 (2004), no. 1, 105-143.

[50] W.-M. Ni, Diffusion, cross-diffusion, and their spike-layer steady states, Notices Amer. Math. Soc. 45 (1998), no. 1, 9-18.

[51] W.-M. Ni, Qualitative properties of solutions to elliptic problems, Stationary partial differential equations. Vol. I, 157-233, Handb. Differ. Equ., North-Holland, Amsterdam, 2004.

[52] W.-M. Ni and I. Takagi, On the shape of least-energy solutions to a semilinear Neumann problem, Comm. Pure Appl. Math. 41 (1991), no. 7, 819-851.

[53] W.-M. Ni and I. Takagi, Locating the peaks of least-energy solutions to a semilinear Neumann problem, Duke Math. J. 70 (1993), no. 2, 247-281.

[54] F. Pacard and M. Ritoré, From constant mean curvature hypersurfaces to the gradient theory of phase transitions, J. Differential Geom. 64 (2003), no. 3, 359-423.

[55] K. Sakamoto, Existence and stability of three-dimensional boundary-interior layers for the Allen-Cahn equation, Taiwanese J. Math. 9 (2005), no. 3, 331-358.

[56] L. Wang, J. Wei and J. Yang, On Ambrosetti-Malchiodi-Ni Conjecture for general hypersurfaces, Comm. Partial Differential Equations, 36 (2011), no. 12, 2117-2161.

[57] J. Wei, On the boundary spike layer solutions to a singularly perturbed Neumann problem, J. Differential Equations 134 (1997), no. 1, 104-133.

[58] J. Wei, On the interior spike layer solutions to a singularly perturbed Neumann problem, Tohoku Math. J. (2) 50 (1998), no. 2, 159-178.

[59] J. Wei and M. Winter, Stationary solutions for the Cahn-Hilliard equation, Ann. Inst. H. Poincaré Anal. Non Linéaire 15 (1998), no. 4, 459-492.

[60] J. Wei, Existence and stability of spikes for the Gierer-Meinhardt system, Handbook of differential equations: stationary partial differential equations. Vol. V, 487-585, Handb. Differ. Equ., Elsevier/North-Holland, Amsterdam, 2008.

[61] J. Wei and J. Yang, Concentration on lines for a singularly perturbed Neumann problem in two-dimensional domains, Indiana Univ. Math. J. 56 (2007), no. 6, 3025-3073.

[62] J. Wei and J. Yang, Toda system and interior clustering line concentration for a singularly perturbed Neumann problem in two dimensional domain, Discrete Contin. Dyn. Syst. 22 (2008), no. 3, 465-508. 